Article

\title{
Long Dimodules and Quasitriangular Weak Hopf Monoids
}

\author{
José Nicanor Alonso Álvarez ${ }^{1}$ (D) José Manuel Fernández Vilaboa ${ }^{2}$ (D) and Ramón González Rodríguez ${ }^{3, *(D)}$ \\ 1 Departamento de Matemáticas, Universidade de Vigo, Campus Universitario Lagoas-Marcosende, \\ E-36280 Vigo, Spain; jnalonso@uvigo.es \\ 2 Departamento de Álxebra, Universidade de Santiago de Compostela, E-15771 Santiago de Compostela, Spain; \\ josemanuel.fernandez@usc.es \\ 3 Departamento de Matemática Aplicada II, Universidade de Vigo, Campus Universitario Lagoas Marcosende, \\ E-36310 Vigo, Spain \\ * Correspondence: rgon@dma.uvigo.es
}

check for

updates

Citation: Alonso Álvarez, J.N.; Fernández Vilaboa, J.M.; González Rodríguez, R. Long Dimodules and Quasitriangular Weak Hopf Monoids. Mathematics 2021, 9, 424. https:// doi.org/10.3390/math9040424

Academic Editors: Juan Ramón García Rozas, Luis Oyonarte Alcalá and Driss Bennis

Received: 9 January 2021

Accepted: 18 February 2021

Published: 21 February 2021

Publisher's Note: MDPI stays neutral with regard to jurisdictional claims in published maps and institutional affiliations.

Copyright: (C) 2021 by the authors. Licensee MDPI, Basel, Switzerland. This article is an open access article distributed under the terms and conditions of the Creative Commons Attribution (CC BY) license (https:/ / creativecommons.org/licenses/by/ $4.0 /)$.

\begin{abstract}
In this paper, we prove that for any pair of weak Hopf monoids $H$ and $B$ in a symmetric monoidal category where every idempotent morphism splits, the category of $H$ - $B$-Long dimodules ${ }_{H}^{B}$ Long is monoidal. Moreover, if $H$ is quasitriangular and $B$ coquasitriangular, we also prove that ${ }_{H}^{B}$ Long is braided. As a consequence of this result, we obtain that if $H$ is triangular and $B$ cotriangular, ${ }_{H}^{B}$ Long is an example of a symmetric monoidal category.
\end{abstract}

Keywords: Braided (symmetric) monoidal category; Long dimodule; (co)quasitriangular weak Hopf monoid

\section{Introduction}

Let $R$ be a commutative fixed ring with unit and let $C$ be the non-strict symmetric monoidal category of $R$-Mod where $\otimes$ denotes the tensor product over $R$. The notion of Long $H$-dimodule for a commutative and cocommutative Hopf algebra $H$ in $\mathrm{C}$ was introduced by Long [1] to study the Brauer group of $H$-dimodule algebras. For two arbitrary Hopf algebras $H$ and $B$ with bijective antipode there exists a well-known connection between the category of left-left $H$-B-Long dimodules, denoted by ${ }_{H}^{B}$ Long, and the category of left-left Yetter-Drinfel'd modules over the Hopf algebra $H \otimes B$, denoted by ${ }_{H \otimes B}^{H \otimes B} Y$. This relation can be formulated in the following way: if $H$ is a quasitriangular and $B$ coquasitriangular, ${ }_{H}^{B}$ Long is a braided monoidal subcategory of ${ }_{H \otimes B}^{H \otimes B}$ YD. As a consequence of this fact, we ensure that under the suitable conditions, Long dimodules provide nontrivial examples of solutions for the Yang-Baxter equation. On the other hand, for a commutative and cocommutative Hopf algebra $H$, the category of left-right $H$ - $H$-Long dimodules, denoted by ${ }_{H}$ Long $^{H}$, is the category of left-right Yetter-Drinfel'd modules over $H$. Then, for all these reasons, it is not unreasonable to assume that there exists an interesting relationship between Long dimodules and the problem of find solutions for the Yang-Baxter equation. Moreover, the previous statement can be extended, as was proved by Militaru in [2], to the problem of find solutions for the $\mathcal{D}$-equation.

The results about the connections between Long dimodules and Yetter-Drinfeld modules can be generalized to Hom-Hopf algebras and to non-associative Hopf structures as for example Hopf quasigroups. In [3], for two monoidal Hom-Hopf algebras $(H, \alpha)$ and $(B, \beta)$ the authors introduce the notion of generalized Hom-Long dimodule and the category of generalized Hom-Long dimodules proving that this category is an example of autonomous category. Also, if $(H, \alpha)$ is quasitriangular and $(B, \beta)$ is coquasitriangular they obtain that the category of generalized Hom-Long dimodules is a braided monoidal subcategory of the category of left-left Yetter-Drinfel'd modules over the monoidal Hom-Hopf algebra $(H \otimes B, \alpha \otimes \beta)$. On the other hand, in [4] (see also [5]) we can find the definition of Long dimodule for Hopf quasigroups and, if $H$ is a quasitriangular Hopf quasigroup and $B$ coquasitriangular Hopf quasigroup, as in the previous settings, the authors prove 
that the category of left-left $H$ - $B$-Long dimodules is a braided monoidal subcategory of the category of Yetter-Drinfel'd modules over the Hopf quasigroup $H \otimes B$.

The main motivation of this paper is to prove that for weak Hopf algebras and Long dimodules associated with them we can obtain similar results to the ones cited in the previous paragraphs. Weak Hopf algebras (or quantum groupoids in the terminology of Nikshych and Vainerman [6]) were introduced by Böhm, Nill and Szlachányi [7] as novel algebraic structures encompassing Hopf algebras and groupoid algebras. The central difference with other associative and coassocitive Hopf objects is the following: The coproduct is not required to preserve the unit, equivalently, the counit is not a monoid morphism. The main motivations to study weak Hopf algebras come from many relevant facts. For example, on one hand, groupoid algebras and their duals provide natural examples of weak Hopf algebras and, on the other hand, weak Hopf algebras have a remarkable connection with some interesting theories, as for example, the theory of algebra extensions, the theory of dynamical twists of Hopf algebras, the theory of quantum field theories, the theory of operator algebras [6] and the theory of fusion categories in characteristic zero [8]. Also, Hayashi's face algebras (see [9]) are relevant examples of weak Hopf algebras and Yamanouchi's generalized Kac algebras [10] are exactly $C^{*}$-weak Hopf algebras with involutive antipode. Finally, for weak Hopf algebras there exists a well-established theory of Yetter-Drinfeld modules (see [11,12]) for which, as in the Hopf algebra setting, the more remarkable property related with the Yang-Baxter equation is the following: If $H$ is a weak Hopf algebra with bijective antipode the category of left-left Yetter-Drinfeld modules over $H$ is braided monoidal. In this case is a remarkable fact that in a different way to the previously cited cases, the tensor product of two Yetter-Drinfeld modules $M$ and $N$ is a subspace of $M \otimes N$ defined by the image of a suitable idempotent $R$-map $\nabla_{M \otimes N}: M \otimes N \rightarrow M \otimes N$.

In this paper, we work in a monoidal setting to ensure a good level of generality. Then, we use monoids, comonoids, weak bimonoids and weak Hopf monoids instead of algebras, coalgebras, weak bialgebras and weak Hopf algebras. Our main results are contained in Sections 3 and 4 . For two weak Hopf monoids $H$ and $B$, in the third section we introduce the category of $H$ - $B$-Long dimodules, denoted as for the category $R$-Mod, by ${ }_{H}^{B}$ Long and we describe in detail the tensor product of this category. In this setting the tensor product is defined as the image of the composition of two idempotent morphisms associated with the module and comodule structure, respectively. The main result is Theorem 1 which states that ${ }_{H}^{B}$ Long is monoidal. Finally, in the fourth section we prove the main result of this paper. As in the cases cited in the previous paragraphs, we obtain that if $H$ is quasitriangular and $B$ coquasitriangular, ${ }_{H}^{B}$ Long is a braided subcategory of ${ }_{H \otimes B}^{H \otimes B} Y D$ (see Theorem 3). Moreover, if $H$ is triangular and $B$ cotriangular, we established that ${ }_{H}^{B}$ Long is symmetric.

\section{Preliminaries}

A monoidal category is a category $\mathrm{C}$ together with a functor $\otimes: \mathrm{C} \times \mathrm{C} \rightarrow \mathrm{C}$, called tensor product, an object $K$ of $C$, called the unit object, and families of natural isomorphisms

$$
\begin{gathered}
a_{M, N, P}:(M \otimes N) \otimes P \rightarrow M \otimes(N \otimes P), \\
r_{M}: M \otimes K \rightarrow M, \quad l_{M}: K \otimes M \rightarrow M,
\end{gathered}
$$

in C, called associativity, right unit and left unit constraints, respectively, satisfying the Pentagon Axiom and the Triangle Axiom, i.e.,

$$
\begin{gathered}
a_{M, N, P \otimes Q} \circ a_{M \otimes N, P, Q}=\left(i d_{M} \otimes a_{N, P, Q}\right) \circ a_{M, N \otimes P, Q} \circ\left(a_{M, N, P} \otimes i d_{Q}\right), \\
\left(i d_{M} \otimes l_{N}\right) \circ a_{M, K, N}=r_{M} \otimes i d_{N},
\end{gathered}
$$

where for each object $X$ in $C, i d_{X}$ denotes the identity morphism of $X$. For simplicity of notation, given objects $M, N, P$ in $C$ and a morphism $f: M \rightarrow N$, we write $P \otimes f$ for $i d_{P} \otimes f$ and $f \otimes P$ for $f \otimes i d_{P}$. 
A monoidal category is called strict if the associativity, right unit and left unit constraints are identities. It is a well-known fact that every non-strict monoidal category is monoidal equivalent to a strict one (see [13]). Then, in general, we can assume without loss of generality that the category is strict and, as a consequence of the quoted equivalence, the results proved in this paper remain valid for every non-strict symmetric monoidal category, what would include for example the categories of vector spaces over a field $\mathbb{F}$, or the one of left modules over a commutative ring $R$. In what follows, for simplicity of notation, given objects $M, N, P$ in $C$ and a morphism $f: M \rightarrow N$, we write $P \otimes f$ for $i d_{P} \otimes f$ and $f \otimes P$ for $f \otimes i d_{P}$.

A braiding for a strict monoidal category $C$ is a natural family of isomorphisms

$$
t_{M, N}: M \otimes N \rightarrow N \otimes M
$$

subject to the conditions

$$
t_{M, N \otimes P}=\left(N \otimes t_{M, P}\right) \circ\left(t_{M, N} \otimes P\right), \quad t_{M \otimes N, P}=\left(t_{M, P} \otimes N\right) \circ\left(M \otimes t_{N, P}\right) .
$$

A strict braided monoidal category is a strict monoidal category with a braiding. Braided monoidal categories were introduced by Joyal and Street (see [14]) motivated by the theory of braids and links in topology. Please note that as a consequence of the definition, the equalities $t_{M, K}=t_{K, M}=i d_{M}$ hold, for all object $M$ of $C$. If the braiding satisfies that $t_{N, M} \circ t_{M, N}=i d_{M \otimes N}$, for all $M, N$ in $C$, we will say that $C$ is symmetric and the braiding will be called a symmetry.

Throughout this paper $\mathrm{C}$ denotes a strict symmetric monoidal category with tensor product $\otimes$, unit object $K$ and natural isomorphism of symmetry $c$. We also assume that in $C$ every idempotent morphism splits, i.e., for any morphism $q: X \rightarrow X$ such that $q \circ q=q$ there exist an object $Z$, called the image of $q$, and morphisms $i: Z \rightarrow X, p: X \rightarrow Z$, such that $q=i \circ p$ and $p \circ i=i d_{Z}$. Please note that $Z, p$ and $i$ are unique up to isomorphism. The categories satisfying this property constitute a broad class that includes, among others, the categories with epi-monic decomposition for morphisms and categories with equalizers or coequalizers. For example, complete bornological spaces is a symmetric monoidal closed category that is not abelian, but it has coequalizers (see [15]). On the other hand, let Hilb be the category whose objects are complex Hilbert spaces and whose morphisms are the continuous linear maps. Then, Hilb is not an abelian and closed category but it is a symmetric monoidal category (see [16]) with coequalizers.

A monoid in $\mathrm{C}$ is a triple $A=\left(A, \eta_{A}, \mu_{A}\right)$ where $A$ is an object in $\mathrm{C}$ and $\eta_{A}: K \rightarrow A$ (unit), $\mu_{A}: A \otimes A \rightarrow A$ (product) are morphisms in C such that $\mu_{A} \circ\left(A \otimes \eta_{A}\right)=i d_{A}=$ $\mu_{A} \circ\left(\eta_{A} \otimes A\right)$ and $\mu_{A} \circ\left(A \otimes \mu_{A}\right)=\mu_{A} \circ\left(\mu_{A} \otimes A\right)$. Given two monoids $A=\left(A, \eta_{A}, \mu_{A}\right)$ and $B=\left(B, \eta_{B}, \mu_{B}\right), f: A \rightarrow B$ is a monoid morphism if $\mu_{B} \circ(f \otimes f)=f \circ \mu_{A}, f \circ \eta_{A}=\eta_{B}$. Also, if $A, B$ are monoids in $C$, the object $A \otimes B$ is a monoid in $C$ where $\eta_{A \otimes B}=\eta_{A} \otimes \eta_{B}$ and $\mu_{A \otimes B}=\left(\mu_{A} \otimes \mu_{B}\right) \circ\left(A \otimes c_{B, A} \otimes B\right)$.

A comonoid in $\mathrm{C}$ is a triple $D=\left(D, \varepsilon_{D}, \delta_{D}\right)$ where $D$ is an object in $C$ and $\varepsilon_{D}: D \rightarrow K$ (counit), $\delta_{D}: D \rightarrow D \otimes D$ (coproduct) are morphisms in C such that $\left(\varepsilon_{D} \otimes D\right) \circ \delta_{D}=i d_{D}=$ $\left(D \otimes \varepsilon_{D}\right) \circ \delta_{D}$ and $\left(\delta_{D} \otimes D\right) \circ \delta_{D}=\left(D \otimes \delta_{D}\right) \circ \delta_{D}$. If $D=\left(D, \varepsilon_{D}, \delta_{D}\right)$ and $E=\left(E, \varepsilon_{E}, \delta_{E}\right)$ are comonoids, $f: D \rightarrow E$ is a comonoid morphism if $(f \otimes f) \circ \delta_{D}=\delta_{E} \circ f, \varepsilon_{E} \circ f=\varepsilon_{D}$. If $D, E$ are comonoids in $C, D \otimes E$ is a comonoid in C where $\varepsilon_{D \otimes E}=\varepsilon_{D} \otimes \varepsilon_{E}$ and $\delta_{D \otimes E}=$ $\left(D \otimes c_{D, E} \otimes E\right) \circ\left(\delta_{D} \otimes \delta_{E}\right)$.

If $A$ is a monoid, $C$ is a comonoid and $f: C \rightarrow A, g: C \rightarrow A$ are morphisms, we define the convolution product by $f * g=\mu_{A} \circ(f \otimes g) \circ \delta_{C}$.

Definition 1. A weak bimonoid $H$ is an object in $\mathrm{C}$ with a monoid structure $\left(H, \eta_{H}, \mu_{H}\right)$ and a comonoid structure $\left(H, \varepsilon_{H}, \delta_{H}\right)$ such that the following axioms hold:

(a1) $\delta_{H} \circ \mu_{H}=\left(\mu_{H} \otimes \mu_{H}\right) \circ \delta_{H \otimes H}$,

(a2) $\varepsilon_{H} \circ \mu_{H} \circ\left(\mu_{H} \otimes H\right)=\left(\varepsilon_{H} \otimes \varepsilon_{H}\right) \circ\left(\mu_{H} \otimes \mu_{H}\right) \circ\left(H \otimes \delta_{H} \otimes H\right)$

$=\left(\varepsilon_{H} \otimes \varepsilon_{H}\right) \circ\left(\mu_{H} \otimes \mu_{H}\right) \circ\left(H \otimes\left(c_{H, H} \circ \delta_{H}\right) \otimes H\right)$, 
(a3) $\left(\delta_{H} \otimes H\right) \circ \delta_{H} \circ \eta_{H}=\left(H \otimes \mu_{H} \otimes H\right) \circ\left(\delta_{H} \otimes \delta_{H}\right) \circ\left(\eta_{H} \otimes \eta_{H}\right)$ $=\left(H \otimes\left(\mu_{H} \circ c_{H, H}\right) \otimes H\right) \circ\left(\delta_{H} \otimes \delta_{H}\right) \circ\left(\eta_{H} \otimes \eta_{H}\right)$.

Moreover, if there exists a morphism $\lambda_{H}: H \rightarrow H$ in $\mathrm{C}$ (called the antipode of $H$ ) satisfying (a4) $i d_{H} * \lambda_{H}=\left(\left(\varepsilon_{H} \circ \mu_{H}\right) \otimes H\right) \circ\left(H \otimes c_{H, H}\right) \circ\left(\left(\delta_{H} \circ \eta_{H}\right) \otimes H\right)$, (a5) $\lambda_{H} * i d_{H}=\left(H \otimes\left(\varepsilon_{H} \circ \mu_{H}\right)\right) \circ\left(c_{H, H} \otimes H\right) \circ\left(H \otimes\left(\delta_{H} \circ \eta_{H}\right)\right)$, (a6) $\lambda_{H} * i d_{H} * \lambda_{H}=\lambda_{H}$, we will say that the weak bimonoid is a weak Hopf monoid.

For any weak bimonoid, if we define the morphisms $\Pi_{H}^{L}$ (target), $\Pi_{H}^{R}$ (source), $\bar{\Pi}_{H}^{L}$ and $\bar{\Pi}_{H}^{R}$ by

$$
\begin{aligned}
\Pi_{H}^{L}= & \left(\left(\varepsilon_{H} \circ \mu_{H}\right) \otimes H\right) \circ\left(H \otimes c_{H, H}\right) \circ\left(\left(\delta_{H} \circ \eta_{H}\right) \otimes H\right), \\
\Pi_{H}^{R}= & \left(H \otimes\left(\varepsilon_{H} \circ \mu_{H}\right)\right) \circ\left(c_{H, H} \otimes H\right) \circ\left(H \otimes\left(\delta_{H} \circ \eta_{H}\right)\right), \\
& \bar{\Pi}_{H}^{L}=\left(H \otimes\left(\varepsilon_{H} \circ \mu_{H}\right)\right) \circ\left(\left(\delta_{H} \circ \eta_{H}\right) \otimes H\right), \\
& \bar{\Pi}_{H}^{R}=\left(\left(\varepsilon_{H} \circ \mu_{H}\right) \otimes H\right) \circ\left(H \otimes\left(\delta_{H} \circ \eta_{H}\right)\right),
\end{aligned}
$$

it is straightforward to show that they are idempotent and the equalities

$$
\begin{aligned}
& \Pi_{H}^{L} \circ \bar{\Pi}_{H}^{L}=\Pi_{H}^{L}, \quad \Pi_{H}^{L} \circ \bar{\Pi}_{H}^{R}=\bar{\Pi}_{H}^{R}, \quad \Pi_{H}^{R} \circ \bar{\Pi}_{H}^{L}=\bar{\Pi}_{H}^{L}, \quad \Pi_{H}^{R} \circ \bar{\Pi}_{H}^{R}=\Pi_{H}^{R}, \\
& \bar{\Pi}_{H}^{L} \circ \Pi_{H}^{L}=\bar{\Pi}_{H}^{L}, \quad \bar{\Pi}_{H}^{L} \circ \Pi_{H}^{R}=\Pi_{H}^{R}, \quad \bar{\Pi}_{H}^{R} \circ \Pi_{H}^{L}=\Pi_{H}^{L}, \quad \bar{\Pi}_{H}^{R} \circ \Pi_{H}^{R}=\bar{\Pi}_{H}^{R},
\end{aligned}
$$
hold.

On the other hand, denote by $H_{L}$ the image of the target morphism $\Pi_{H}^{L}$ and let $p_{H}^{L}: H \rightarrow H_{L}, i_{H}^{L}: H_{L} \rightarrow H$ be the morphisms such that $i_{H}^{L} \circ p_{H}^{L}=\Pi_{H}^{L}$ and $p_{H}^{L} \circ i_{H}^{L}=i d_{H_{L}}$. Then,

$$
\left(H_{L}, \eta_{H_{L}}=p_{H}^{L} \circ \eta_{H}, \mu_{H_{L}}=p_{H}^{L} \circ \mu_{H} \circ\left(i_{H}^{L} \otimes i_{H}^{L}\right)\right)
$$

is a monoid and

$$
\left(H_{L}, \varepsilon_{H_{L}}=\varepsilon_{H} \circ i_{H}^{L}, \delta_{H_{L}}=\left(p_{H}^{L} \otimes p_{H}^{L}\right) \circ \delta_{H} \circ i_{H}^{L}\right)
$$

is a comonoid. The morphisms $\eta_{H_{L}}, \mu_{H_{L}}, \varepsilon_{H_{L}}$ and $\delta_{H_{L}}$ are the unique morphisms satisfying

$$
\begin{gathered}
i_{H}^{L} \circ \eta_{H_{L}}=\eta_{H}, \quad i_{H}^{L} \circ \mu_{H_{L}}=\mu_{H} \circ\left(i_{H}^{L} \otimes i_{H}^{L}\right), \\
\varepsilon_{H_{L}} \circ p_{H}^{L}=\varepsilon_{H}, \quad \delta_{H_{L}} \circ p_{H}^{L}=\left(p_{H}^{L} \otimes p_{H}^{L}\right) \circ \delta_{H},
\end{gathered}
$$

respectively.

Now we summarize the main properties of the idempotent morphisms $\Pi_{H}^{L}, \Pi_{H}^{R}, \bar{\Pi}_{H}^{L}$ and $\bar{\Pi}_{H}^{R}$ (see [17] for the detailed proofs).

$$
\begin{array}{cc}
\Pi_{H}^{L} \circ \mu_{H} \circ\left(H \otimes \Pi_{H}^{L}\right)=\Pi_{H}^{L} \circ \mu_{H}, & \Pi_{H}^{R} \circ \mu_{H} \circ\left(\Pi_{H}^{R} \otimes H\right)=\Pi_{H}^{R} \circ \mu_{H}, \\
\bar{\Pi}_{H}^{L} \circ \mu_{H} \circ\left(H \otimes \bar{\Pi}_{H}^{L}\right)=\bar{\Pi}_{H}^{L} \circ \mu_{H}, & \bar{\Pi}_{H}^{R} \circ \mu_{H} \circ\left(\bar{\Pi}_{H}^{R} \otimes H\right)=\bar{\Pi}_{H}^{R} \circ \mu_{H}, \\
\left(H \otimes \Pi_{H}^{L}\right) \circ \delta_{H} \circ \Pi_{H}^{L}=\delta_{H} \circ \Pi_{H}^{L}, & \left(\Pi_{H}^{R} \otimes H\right) \circ \delta_{H} \circ \Pi_{H}^{R}=\delta_{H} \circ \Pi_{H}^{R}, \\
\left(H \otimes \bar{\Pi}_{H}^{R}\right) \circ \delta_{H} \circ \bar{\Pi}_{H}^{R}=\delta_{H} \circ \bar{\Pi}_{H}^{R}, & \left(\bar{\Pi}_{H}^{L} \otimes H\right) \circ \delta_{H} \circ \bar{\Pi}_{H}^{L}=\delta_{H} \circ \bar{\Pi}_{H}^{L}, \\
\mu_{H} \circ\left(H \otimes \Pi_{H}^{L}\right)=\left(\left(\varepsilon_{H} \circ \mu_{H}\right) \otimes H\right) \circ\left(H \otimes c_{H, H}\right) \circ\left(\delta_{H} \otimes H\right),
\end{array}
$$




$$
\begin{gathered}
\left(H \otimes \Pi_{H}^{L}\right) \circ \delta_{H}=\left(\mu_{H} \otimes H\right) \circ\left(H \otimes c_{H, H}\right) \circ\left(\left(\delta_{H} \circ \eta_{H}\right) \otimes H\right), \\
\mu_{H} \circ\left(\Pi_{H}^{R} \otimes H\right)=\left(H \otimes\left(\varepsilon_{H} \circ \mu_{H}\right)\right) \circ\left(c_{H, H} \otimes H\right) \circ\left(H \otimes \delta_{H}\right) \\
\left(\Pi_{H}^{R} \otimes H\right) \circ \delta_{H}=\left(H \otimes \mu_{H}\right) \circ\left(c_{H, H} \otimes H\right) \circ\left(H \otimes\left(\delta_{H} \circ \eta_{H}\right)\right) \\
\mu_{H} \circ\left(\bar{\Pi}_{H}^{R} \otimes H\right)=\left(\left(\varepsilon_{H} \circ \mu_{H}\right) \otimes H\right) \circ\left(H \otimes \delta_{H}\right), \\
\mu_{H} \circ\left(H \otimes \bar{\Pi}_{H}^{L}\right)=\left(H \otimes\left(\varepsilon_{H} \circ \mu_{H}\right)\right)\left(\delta_{H} \otimes H\right), \\
\left(\bar{\Pi}_{H}^{L} \otimes H\right) \circ \delta_{H}=\left(H \otimes \mu_{H}\right) \circ\left(\left(\delta_{H} \circ \eta_{H}\right) \otimes H\right), \\
\left(H \otimes \bar{\Pi}_{H}^{R}\right) \circ \delta_{H}=\left(\mu_{H} \otimes H\right) \circ\left(H \otimes\left(\delta_{H} \circ \eta_{H}\right)\right), \\
\delta_{H} \circ \eta_{H}=\left(\Pi_{H}^{R} \otimes H\right) \circ \delta_{H} \circ \eta_{H}=\left(H \otimes \Pi_{H}^{L}\right) \circ \delta_{H} \circ \eta_{H}=\left(H \otimes \bar{\Pi}_{H}^{R}\right) \circ \delta_{H} \circ \eta_{H} \\
=\left(\bar{\Pi}_{H}^{L} \otimes H\right) \circ \delta_{H} \circ \eta_{H}, \\
\varepsilon_{H} \circ \mu_{H}=\varepsilon_{H} \circ \mu_{H} \circ\left(\Pi_{H}^{R} \otimes H\right)=\varepsilon_{H} \circ \mu_{H} \circ\left(H \otimes \Pi_{H}^{L}\right)=\varepsilon_{H} \circ \mu_{H} \circ\left(\bar{\Pi}_{H}^{R} \otimes H\right) \\
=\varepsilon_{H} \circ \mu_{H} \circ\left(H \otimes \bar{\Pi}_{H}^{L}\right) .
\end{gathered}
$$

Lemma 1. Let $H$ be a weak bimonoid in $\mathrm{C}$. The following identities hold:

$$
\begin{gathered}
\bar{\Pi}_{H}^{R} \circ \mu_{H} \circ\left(H \otimes \Pi_{H}^{L}\right)=\Pi_{H}^{L} \circ \mu_{H} \circ\left(\bar{\Pi}_{H}^{R} \otimes H\right), \\
\left(\bar{\Pi}_{H}^{L} \otimes H\right) \circ \delta_{H} \circ \Pi_{H}^{L}=\left(H \otimes \Pi_{H}^{L}\right) \circ \delta_{H} \circ \bar{\Pi}_{H}^{L}, \\
\left(H \otimes \mu_{H}\right) \circ\left(\left(\left(H \otimes \Pi_{H}^{L}\right) \circ \delta_{H} \circ \bar{\Pi}_{H}^{L}\right) \otimes H\right) \circ \delta_{H}=\left(\bar{\Pi}_{H}^{L} \otimes H\right) \circ \delta_{H}, \\
\mu_{H} \circ\left(\left(\bar{\Pi}_{H}^{R} \circ \mu_{H} \circ\left(H \otimes \Pi_{H}^{L}\right)\right) \otimes H\right) \circ\left(H \otimes \delta_{H}\right)=\mu_{H} \circ\left(\bar{\Pi}_{H}^{R} \otimes H\right) .
\end{gathered}
$$

Proof. The proof for (19) is the following:

$$
\bar{\Pi}_{H}^{R} \circ \mu_{H} \circ\left(H \otimes \Pi_{H}^{L}\right)
$$

$=\left(\left(\varepsilon_{H} \circ \mu_{H}\right) \otimes H\right) \circ\left(H \otimes c_{H, H}\right) \circ\left(\left(\left(H \otimes \bar{\Pi}_{H}^{R}\right) \circ \delta_{H}\right) \otimes H\right)$ (by (9) and the naturality of $\left.c\right)$

$=\left(\left(\varepsilon_{H} \circ \mu_{H}\right) \otimes H\right) \circ\left(\mu_{H} \otimes c_{H, H}\right) \circ\left(H \otimes\left(\delta_{H} \circ \eta_{H}\right) \otimes H\right)($ by (16))

$=\left(\left(\varepsilon_{H} \circ \mu_{H}\right) \otimes H\right) \circ\left(H \otimes\left(\left(\mu_{H} \otimes H\right) \circ\left(H \otimes c_{H, H}\right) \circ\left(\left(\delta_{H} \circ \eta_{H}\right) \otimes H\right)\right)\right)$ (by associativity of $\left.\mu_{H}\right)$

$=\left(\left(\varepsilon_{H} \circ \mu_{H}\right) \otimes \Pi_{H}^{L}\right) \circ\left(H \otimes \delta_{H}\right)($ by (10))

$=\Pi_{H}^{L} \circ \mu_{H} \circ\left(\bar{\Pi}_{H}^{R} \otimes H\right)$ (by (13)).

On the other hand,

$$
\left(\bar{\Pi}_{H}^{L} \otimes H\right) \circ \delta_{H} \circ \Pi_{H}^{L}
$$

$=\left(H \otimes \mu_{H}\right) \circ\left(\left(\delta_{H} \circ \eta_{H}\right) \otimes \Pi_{H}^{L}\right)($ by (15))

$=\left(H \otimes\left(\left(\left(\varepsilon_{H} \circ \mu_{H}\right) \otimes H\right) \circ\left(H \otimes c_{H, H}\right) \circ\left(\delta_{H} \otimes H\right)\right)\right) \circ\left(\left(\delta_{H} \circ \eta_{H}\right) \otimes H\right)$ (by (9))

$=\left(H \otimes\left(\varepsilon_{H} \circ \mu_{H}\right) \otimes H\right) \circ\left(\delta_{H} \otimes c_{H, H}\right) \circ\left(\left(\delta_{H} \circ \eta_{H}\right) \otimes H\right)$ (by coassociativity of $\left.\delta_{H}\right)$

$=\left(\left(\mu_{H} \circ\left(H \otimes \bar{\Pi}_{H}^{L}\right)\right) \otimes H\right) \circ\left(H \otimes c_{H, H}\right) \circ\left(\left(\delta_{H} \circ \eta_{H}\right) \otimes H\right)$ (by (14))

$=\left(H \otimes \Pi_{H}^{L}\right) \circ \delta_{H} \circ \bar{\Pi}_{H}^{L}$ (by the naturality of $c$ and $\left.(10)\right)$,

and (20) holds.

The proof for (21) is the following:

$\left(H \otimes \mu_{H}\right) \circ\left(\left(\left(H \otimes \Pi_{H}^{L}\right) \circ \delta_{H} \circ \bar{\Pi}_{H}^{L}\right) \otimes H\right) \circ \delta_{H}$

$=\left(H \otimes \mu_{H}\right) \circ\left(\left(\left(\mu_{H} \otimes \mu_{H}\right) \circ \delta_{H \otimes H} \circ\left(\eta_{H} \otimes \eta_{H}\right)\right) \otimes H\right)$ (by (10), (15) and associativity of $\left.\mu_{H}\right)$

$\left.=\left(H \otimes \mu_{H}\right) \circ\left(\delta_{H} \circ \mu_{H} \circ\left(\eta_{H} \otimes \eta_{H}\right)\right) \otimes H\right)$ (by (a1) of Definition 1)

$=\left(\bar{\Pi}_{H}^{L} \otimes H\right) \circ \delta_{H}($ by (15)) . 
Finally, the proof for (22) can be obtained as the previous one by reversing arrows.

If $H$ is a weak Hopf monoid in C, the antipode $\lambda_{H}$ is unique, antimultiplicative, anticomultiplicative and leaves the unit and the counit invariant:

$$
\begin{gathered}
\lambda_{H} \circ \mu_{H}=\mu_{H} \circ\left(\lambda_{H} \otimes \lambda_{H}\right) \circ c_{H, H}, \quad \delta_{H} \circ \lambda_{H}=c_{H, H} \circ\left(\lambda_{H} \otimes \lambda_{H}\right) \circ \delta_{H}, \\
\lambda_{H} \circ \eta_{H}=\eta_{H}, \quad \varepsilon_{H} \circ \lambda_{H}=\varepsilon_{H} .
\end{gathered}
$$

Also, it is straightforward to show the equalities

$$
\begin{array}{cl}
\Pi_{H}^{L}=i d_{H} * \lambda_{H}, & \Pi_{H}^{R}=\lambda_{H} * i d_{H}, \\
\Pi_{H}^{L} * i d_{H}=i d_{H}=i d_{H} * \Pi_{H}^{R}, & \Pi_{H}^{R} * \lambda_{H}=\lambda_{H}=\lambda_{H} * \Pi_{H}^{L}, \\
\Pi_{H}^{L} * \Pi_{H}^{L}=\Pi_{H}^{L}, & \Pi_{H}^{R} * \Pi_{H}^{R}=\Pi_{H}^{R}
\end{array}
$$

and

$$
\Pi_{H}^{L}=\lambda_{H} \circ \bar{\Pi}_{H}^{L}=\bar{\Pi}_{H}^{R} \circ \lambda_{H}, \quad \Pi_{H}^{R}=\bar{\Pi}_{H}^{L} \circ \lambda_{H}=\lambda_{H} \circ \bar{\Pi}_{H}^{R} .
$$

If $H$ and $B$ are weak bimonoids in $C$, the tensor product $H \otimes B$ so is. In this case, the monoid-comonoid structure is the one of $H \otimes B$ and

$$
\Pi_{H \otimes B}^{L}=\Pi_{H}^{L} \otimes \Pi_{B}^{L}, \quad \Pi_{H \otimes B}^{R}=\Pi_{H}^{R} \otimes \Pi_{B}^{R} .
$$

Then, if $H$ and $B$ are weak Hopf monoids in $C$, the tensor product $H \otimes B$ so is with $\lambda_{H \otimes B}=\lambda_{H} \otimes \lambda_{B}$. Please note that

$$
(H \otimes B)_{L}=H_{L} \otimes B_{L} .
$$

Finally, for any weak bimonoid $H$, we can define the opposite and coopposite weak bimonoids as $H^{o p}=\left(H, \eta_{H}, \mu_{H} \circ c_{H, H}, \varepsilon_{H}, \delta_{H}\right)$ and $H^{\text {coop }}=\left(H, \eta_{H}, \mu_{H}, \varepsilon_{H}, c_{H, H} \circ \delta_{H}\right)$, respectively. If $H$ is a weak Hopf monoid and the antipode is an isomorphism, $H^{o p}=$ $\left(H, \eta_{H}, \mu_{H} \circ c_{H, H}, \varepsilon_{H}, \delta_{H}, \lambda_{H}^{-1}\right)$ and $H^{c o o p}=\left(H, \eta_{H}, \mu_{H}, \varepsilon_{H}, c_{H, H} \circ \delta_{H}, \lambda_{H}^{-1}\right)$, are weak Hopf monoids.

In the end of this section, we summarize some properties about left modules and left comodules over a weak Hopf monoid. The complete details can be found in [18-20].

Definition 2. Let $H$ be a weak Hopf monoid in $C$. We say that $\left(M, \varphi_{M}\right)$ is a left $H$-module if $M$ is an object in $\mathrm{C}$ and $\varphi_{M}: H \otimes M \rightarrow M$ is a morphism in $\mathrm{C}$ such that

$$
\varphi_{M} \circ\left(\eta_{H} \otimes M\right)=i d_{M}, \quad \varphi_{M} \circ\left(H \otimes \varphi_{M}\right)=\varphi_{M} \circ\left(\mu_{H} \otimes M\right) .
$$

Given two left $H$-modules $\left(M, \varphi_{M}\right)$ and $\left(N, \varphi_{N}\right)$, a morphism $f: M \rightarrow N$ in $\mathrm{C}$ is a morphism of left $H$-modules if

$$
\varphi_{N} \circ(H \otimes f)=f \circ \varphi_{M} .
$$

If $\left(M, \varphi_{M}\right)$ and $\left(N, \varphi_{N}\right)$ are left $H$-modules we define the morphism $\varphi_{M \otimes N}: H \otimes M \otimes N \rightarrow$ $M \otimes N$ as

$$
\varphi_{M \otimes N}=\left(\varphi_{M} \otimes \varphi_{N}\right) \circ\left(H \otimes c_{H, M} \otimes N\right) \circ\left(\delta_{H} \otimes M \otimes N\right) .
$$

It is easy to show that $\varphi_{M \otimes N}$ satisfies

$$
\varphi_{M \otimes N} \circ\left(H \otimes \varphi_{M \otimes N}\right)=\varphi_{M \otimes N} \circ\left(\mu_{H} \otimes M \otimes N\right)
$$

and the morphism

$$
\nabla_{M \otimes N}=\varphi_{M \otimes N} \circ\left(\eta_{H} \otimes M \otimes N\right): M \otimes N \rightarrow M \otimes N
$$


is an idempotent. If we denote by $M \bullet N$ the image of $\nabla_{M \otimes N}$ and by $p_{M \otimes N}: M \otimes N \rightarrow$ $M \boxminus N, i_{M \otimes N}: M \boxminus N \rightarrow M \otimes N$ the morphisms such that $i_{M \otimes N} \circ p_{M \otimes N}=\nabla_{M \otimes N}$ and $p_{M \otimes N} \circ i_{M \otimes N}=i d_{M \boxminus N}$, it is not difficult to see that the object $M \square N$ is a left H-module with action

$$
\varphi_{M \boxminus N}=p_{M \otimes N} \circ \varphi_{M \otimes N} \circ\left(H \otimes i_{M \otimes N}\right): H \otimes M \boxminus N \rightarrow M \boxminus N
$$

and the equalities

$$
\varphi_{M \otimes N} \circ\left(H \otimes \nabla_{M \otimes N}\right)=\varphi_{M \otimes N}=\nabla_{M \otimes N} \circ \varphi_{M \otimes N},
$$

hold. Moreover, if $\left(M, \varphi_{M}\right),\left(N, \varphi_{N}\right)$ and $\left(P, \varphi_{P}\right)$ are left $H$-modules, we have that

$$
\left(M \otimes \nabla_{N \otimes P}\right) \circ\left(\nabla_{M \otimes N} \otimes P\right)=\left(\nabla_{M \otimes N} \otimes P\right) \circ\left(M \otimes \nabla_{N \otimes P}\right)
$$

also holds.

If $f:\left(M, \varphi_{M}\right) \rightarrow\left(M^{\prime}, \varphi_{M^{\prime}}\right)$ and $g:\left(N, \varphi_{N}\right) \rightarrow\left(N^{\prime}, \varphi_{N^{\prime}}\right)$ are morphisms of left Hmodules, then,

$$
f \boxminus g=p_{M^{\prime} \otimes N^{\prime}} \circ(f \otimes g) \circ i_{M \otimes N}: M \boxminus N \rightarrow M^{\prime} \bullet N^{\prime}
$$

is a morphism of left $H$-modules between $\left(M \boxminus N, \varphi_{M \square N}\right)$ and $\left(M^{\prime} \boxminus N^{\prime}, \varphi_{M^{\prime} \bullet N^{\prime}}\right)$. Moreover,

$$
(f \otimes g) \circ \nabla_{M \otimes N}=\nabla_{M^{\prime} \otimes N^{\prime}} \circ(f \otimes g) .
$$

Definition 3. We say that $\left(M, \rho_{M}\right)$ is a left $H$-comodule in $\mathrm{C}$ if $M$ is an object in $\mathrm{C}$ and $\rho_{M}$ : $M \rightarrow H \otimes M$ is a morphism in $\mathrm{C}$ such that

$$
\left(\varepsilon_{H} \otimes M\right) \circ \rho_{M}=i d_{M,} \quad\left(H \otimes \rho_{M}\right) \circ \rho_{M}=\left(\delta_{H} \otimes M\right) \circ \rho_{M} .
$$

Given two left $H$-comodules $\left(M, \rho_{M}\right)$ and $\left(N, \rho_{N}\right)$, a morphism $f: M \rightarrow N$ in $C$ is a morphism of left $\mathrm{H}$-comodules if

$$
\rho_{N} \circ f=(H \otimes f) \circ \rho_{M}
$$

If $\left(M, \rho_{M}\right)$ and $\left(N, \rho_{N}\right)$ are left $H$-comodules we define the morphism $\rho_{M \otimes N}: M \otimes N \rightarrow$ $H \otimes M \otimes N$ as

$$
\rho_{M \otimes N}=\left(\mu_{H} \otimes M \otimes N\right) \circ\left(H \otimes c_{M, H} \otimes N\right) \circ\left(\rho_{M} \otimes \rho_{N}\right) .
$$

It is easy to show that $\rho_{M \otimes N}$ satisfies

$$
\left(H \otimes \rho_{M \otimes N}\right) \circ \rho_{M \otimes N}=\left(\delta_{H} \otimes M \otimes N\right) \circ \rho_{M \otimes N}
$$

and the morphism

$$
\nabla_{M \otimes N}^{\prime}=\left(\varepsilon_{H} \otimes M \otimes N\right) \circ \rho_{M \otimes N}: M \otimes N \rightarrow M \otimes N
$$

is an idempotent. If we denote by $M \odot N$ the image of $\nabla_{M \otimes N}^{\prime}$ and by $p_{M \otimes N}^{\prime}: M \otimes N \rightarrow$ $M \odot N, i_{M \otimes N}^{\prime}: M \odot N \rightarrow M \otimes N$ the morphisms such that $i_{M \otimes N}^{\prime} \circ p_{M \otimes N}^{\prime}=\nabla_{M \otimes N}^{\prime}$ and $p_{M \otimes N}^{\prime} \circ i_{M \otimes N}^{\prime}=i d_{M \odot N}$, it is not difficult to see that the object $M \odot N$ is a left H-comodule with coaction

$$
\rho_{M \odot N}=\left(H \otimes p_{M \otimes N}^{\prime}\right) \circ \rho_{M \otimes N} \circ i_{M \otimes N}^{\prime}: M \odot N \rightarrow H \otimes(M \odot N)
$$

and the equality

$$
\left(H \otimes \nabla_{M \otimes N}^{\prime}\right) \circ \rho_{M \otimes N}=\rho_{M \otimes N}=\rho_{M \otimes N} \circ \nabla_{M \otimes N}^{\prime}
$$


holds. Moreover, as in the case of left modules, if $\left(M, \rho_{M}\right),\left(N, \rho_{N}\right)$ and $\left(P, \rho_{P}\right)$ are left $H$ comodules, we have that

$$
\left(M \otimes \nabla_{N \otimes P}^{\prime}\right) \circ\left(\nabla_{M \otimes N}^{\prime} \otimes P\right)=\left(\nabla_{M \otimes N}^{\prime} \otimes P\right) \circ\left(M \otimes \nabla_{N \otimes P}^{\prime}\right)
$$

also holds.

If $f:\left(M, \rho_{M}\right) \rightarrow\left(M^{\prime}, \rho_{M^{\prime}}\right)$ and $g:\left(N, \rho_{N}\right) \rightarrow\left(N^{\prime}, \rho_{N^{\prime}}\right)$ are morphisms of left $H$ comodules, then,

$$
f \odot g=p_{M^{\prime} \times N^{\prime}}^{\prime} \circ(f \otimes g) \circ i_{M \times N}^{\prime}: M \odot N \rightarrow M^{\prime} \odot N^{\prime}
$$

is a morphism of left $H$-comodules between $\left(M \odot N, \rho_{M \odot N}\right)$ and $\left(M^{\prime} \odot N^{\prime}, \rho_{M^{\prime} \odot N^{\prime}}\right)$. Moreover,

$$
(f \otimes g) \circ \nabla_{M \otimes N}^{\prime}=\nabla_{M^{\prime} \otimes N^{\prime}}^{\prime} \circ(f \otimes g) .
$$

\section{The Category of Long Dimodules Over Weak Hopf Monoids}

In this section, we generalize the notion of Long dimodule to the weak Hopf monoid setting.

Definition 4. Let $H$ and $B$ be weak Hopf monoids in C. A left-left $H$-B-Long dimodule $\left(M, \varphi_{M}, \rho_{M}\right)$ is both a left $H$-module with action $\varphi_{M}: H \otimes M \rightarrow M$ and a left B-comodule with coaction $\rho_{M}: M \rightarrow B \otimes M$ such that the equality

$$
\rho_{M} \circ \varphi_{M}=\left(B \otimes \varphi_{M}\right) \circ\left(c_{H, B} \otimes M\right) \circ\left(H \otimes \rho_{M}\right)
$$

holds.

A morphism between two left-left $H$-B-Long dimodules $\left(M, \varphi_{M}, \rho_{M}\right)$ and $\left(N, \varphi_{N}, \rho_{N}\right)$ is a morphism $f: M \rightarrow N$ of left H-modules and left B-comodules. Left-left $H$-B-Long dimodules and morphism of left-left $H$-B-Long dimodules form a category, denoted as ${ }_{H}^{B}$ Long.

In a similar way we can define left-right, right-left and right-right $H$-B-Long dimodules and we have the categories ${ }_{H}$ Long $^{B},{ }^{B}$ Long $_{H}$ and Long $_{H}^{B}$, respectively.

Below we will give examples of left-left $H$ - $B$-Long dimodules. We want to highlight that if the antipodes of $H$ and $B$ are isomorphisms, it is possible to give many more considering opposite and coopposite weak Hopf monoids.

Example 1. Let $H$ and $B$ be weak Hopf monoids in $\mathrm{C}$. The triple

$$
\left(H \otimes B, \varphi_{H \otimes B}=\mu_{H} \otimes B, \rho_{H \otimes B}=\left(c_{H, B} \otimes B\right) \circ\left(H \otimes \delta_{B}\right)\right)
$$

is in ${ }_{H}^{B}$ Long.

Similarly, if $H_{L}$ and $B_{L}$ are the images of the target morphisms with the corresponding structure of monoid-comonoid, by the properties of $\eta_{H}$ and $\varepsilon_{B}$, (5) for $H$, (7) for B, the associativity of $\mu_{H}$, the coassociativity of $\delta_{B}$ and the naturality of $c$, we have that

$\left(H_{L} \otimes B_{L}, \varphi_{H_{L} \otimes B_{L}}=\left(p_{H}^{L} \circ \mu_{H} \circ\left(H \otimes i_{H}^{L}\right)\right) \otimes B_{L}, \rho_{H_{L} \otimes B_{L}}=\left(c_{H_{L}, B} \otimes p_{B}^{L}\right) \circ\left(H_{L} \otimes\left(\delta_{B} \circ i_{B}^{L}\right)\right)\right.$ belongs to ${ }_{H}^{B}$ Long.

Finally, let $\left(M, \varphi_{M}\right)$ be a left $H$-module. Then, it is easy to show that

$$
\left(B \otimes M, \varphi_{B \otimes M}=\left(B \otimes \varphi_{M}\right) \circ\left(c_{H, B} \otimes M\right), \rho_{B \otimes M}=\delta_{B} \otimes M\right)
$$

is an example of left-left $H$-B-Long dimodule. Moreover, if $\left(M, \rho_{M}\right)$ be a left B-comodule,

$$
\left(H \otimes M, \varphi_{H \otimes M}=\mu_{H} \otimes M, \rho_{H \otimes M}=\left(c_{H, B} \otimes M\right) \circ\left(H \otimes \rho_{M}\right)\right)
$$

belongs to the category ${ }_{H}^{B}$ Long. 
Example 2. Let $H$ and $B$ be weak Hopf monoids in $C$. A skew pairing between $H$ and $B$ over $K$ is a morphism $\tau: H \otimes B \rightarrow K$ such that the equalities

(b1) $\tau \circ\left(\mu_{H} \otimes B\right)=(\tau \otimes \tau) \circ\left(H \otimes c_{H, B} \otimes B\right) \circ\left(H \otimes H \otimes \delta_{B}\right)$,

(b2) $\tau \circ\left(H \otimes \mu_{B}\right)=(\tau \otimes \tau) \circ\left(H \otimes c_{H, B} \otimes B\right) \circ\left(\left(c_{H, H} \circ \delta_{H}\right) \otimes B \otimes B\right)$,

(b3) $\tau \circ\left(\eta_{H} \otimes B\right)=\varepsilon_{B}$,

(b4) $\tau \circ\left(H \otimes \eta_{B}\right)=\varepsilon_{H}$,

hold.

Let $\left(M, \rho_{M}\right)$ be a left $B$-comodule and let $\tau: H \otimes B \rightarrow K$ be a skew pairing between $H$ and $B$ over $K$ such that

$$
(\tau \otimes B) \circ\left(H \otimes \delta_{B}\right)=(B \otimes \tau) \circ\left(c_{H, B} \otimes B\right) \circ\left(H \otimes \delta_{B}\right) .
$$

Then, the triple

$$
\left(M, \varphi_{M}=(\tau \otimes M) \circ\left(\lambda_{H} \otimes \rho_{M}\right), \rho_{M}\right)
$$

is in ${ }_{H}^{B}$ Long. Indeed, by (24), (b3) and the B-comodule condition for $M$, we have that $\varphi_{M} \circ\left(\eta_{H} \otimes\right.$ $M)=i d_{M}$. Moreover, using that $M$ is a left B-comodule, the naturality of $c$, (b1) and (23),

$$
\begin{aligned}
& \varphi_{M} \circ\left(H \otimes \varphi_{M}\right) \\
= & \left(\left((\tau \otimes \tau) \circ\left(H \otimes c_{H, B} \otimes B\right) \circ\left(H \otimes H \otimes \delta_{B}\right)\right) \otimes M\right) \circ\left(\left(c_{H, H} \circ\left(\lambda_{H} \otimes \lambda_{H}\right)\right) \otimes \rho_{M}\right) \\
= & \left(\left(\tau \circ\left(\mu_{H} \otimes B\right)\right) \otimes M\right) \circ\left(\left(c_{H, H} \circ\left(\lambda_{H} \otimes \lambda_{H}\right)\right) \otimes \rho_{M}\right) \\
= & \varphi_{M} \circ\left(\mu_{H} \otimes M\right),
\end{aligned}
$$

holds and, consequently, $\left(M, \varphi_{M}\right)$ is a left $H$-module. Finally, using that $M$ is a left B-comodule and (45), condition (44) holds because

$$
\begin{aligned}
& \rho_{M} \circ \varphi_{M} \\
= & \left(\left((\tau \otimes B) \circ\left(H \otimes \delta_{B}\right)\right) \otimes M\right) \circ\left(\lambda_{H} \otimes \rho_{M}\right) \\
= & \left(\left((B \otimes \tau) \circ\left(c_{H, B} \otimes B\right) \circ\left(H \otimes \delta_{B}\right)\right) \otimes M\right) \circ\left(\lambda_{H} \otimes \rho_{M}\right) \\
= & \left(B \otimes \varphi_{M}\right) \circ\left(c_{H, B} \otimes M\right) \circ\left(H \otimes \rho_{M}\right) .
\end{aligned}
$$

In particular, if $H=B, \rho_{H}=\delta_{H}$ and $\tau: H \otimes H \rightarrow K$ is a skew pairing between $H$ and $H$ over K such that

$$
(\tau \otimes H) \circ\left(H \otimes \delta_{H}\right)=(H \otimes \tau) \circ\left(c_{H, H} \otimes H\right) \circ\left(H \otimes \delta_{H}\right) .
$$

we obtain that $\left(H, \varphi_{H}=(\tau \otimes M) \circ\left(\lambda_{H} \otimes \delta_{H}\right), \delta_{H}\right)$ is in ${ }_{H}^{H}$ Long. Moreover, if $\lambda_{H}$ is an isomorphism, the triple $\left(H, \varphi_{H}, \delta_{H}\right)$ belongs to ${ }_{H}^{H}$ Long if and only if (46) holds.

Example 3. Let $H$ and $B$ be weak Hopf monoids in $\mathrm{C}$. We define a skew copairing between $H$ and $B$ over $K$ as a morphism $\sigma: K \rightarrow H \otimes B$ such that the equalities

$(c 1)\left(\delta_{H} \otimes B\right) \circ \sigma=\left(H \otimes H \otimes \mu_{B}\right) \circ\left(H \otimes c_{B, H} \otimes B\right) \circ(\sigma \otimes \sigma)$,

(c2) $\left(H \otimes \delta_{B}\right) \circ \sigma=\left(\left(\mu_{H} \circ c_{H, H}\right) \otimes B \otimes B\right) \circ\left(H \otimes c_{B, H} \otimes B\right) \circ(\sigma \otimes \sigma)$,

(c3) $\left(\varepsilon_{H} \otimes B\right) \circ \sigma=\eta_{B}$,

(c4) $\left(H \otimes \varepsilon_{B}\right) \circ \sigma=\eta_{H}$,

hold.

Now, let $\left(M, \varphi_{M}\right)$ be a left $H$-module and let $\sigma: K \rightarrow H \otimes B$ be a skew copairing between $H$ and $B$ over $K$ such that

$$
\left(\mu_{H} \otimes B\right) \circ(H \otimes \sigma)=\left(\left(\mu_{H} \circ c_{H, H}\right) \otimes B\right) \circ(H \otimes \sigma) .
$$
have that

Then, by a similar proof to the one developed for the example linked to skew pairings we

$$
\left(M, \varphi_{M}, \rho_{M}=\left(B \otimes \varphi_{M}\right) \circ\left(\left(c_{H, B} \circ \sigma\right) \otimes M\right)\right)
$$

belongs to ${ }_{H}^{B}$ Long. Indeed: First note that by the naturality of $c,(c 4)$ and the condition of lef $H$-module for $M$, we have that $\left(\varepsilon_{B} \otimes M\right) \circ \rho_{M}=i d_{M}$. Moreover, using that $M$ is a left $H$-module, (c2) and the naturality of $c$, 


$$
\begin{aligned}
& \left(B \otimes \rho_{M}\right) \circ \rho_{M} \\
= & \left(B \otimes B \otimes \varphi_{M}\right) \circ\left(B \otimes c_{H, B} \otimes M\right) \circ\left(c_{H, B} \otimes B \otimes M\right) \circ\left(\left(\left(\mu_{H} \circ c_{H, H}\right) \otimes B \otimes B\right)\right. \\
& \left.\left.\circ\left(H \otimes c_{B, H} \otimes B\right) \circ(\sigma \otimes \sigma)\right) \otimes M\right) \\
= & \left(B \otimes B \otimes \varphi_{M}\right) \circ\left(B \otimes c_{H, B} \otimes M\right) \circ\left(c_{H, B} \otimes B \otimes M\right) \circ\left(\left(\left(H \otimes \delta_{B}\right) \circ \sigma\right) \otimes M\right) \\
= & \left(\delta_{B} \otimes M\right) \circ \rho_{M},
\end{aligned}
$$

and then, $\left(M, \rho_{M}\right)$ is a left B-comodule. Finally, using (47), the naturality of $c$ and the condition of left $H$-module for $M$, (44) holds because

$$
\begin{aligned}
& \rho_{M} \circ \varphi_{M} \\
= & \left(B \otimes \varphi_{M}\right) \circ\left(c_{H, B} \otimes M\right) \circ\left(\left(\left(\left(\mu_{H} \circ c_{H, H}\right) \otimes B\right) \circ(H \otimes \sigma)\right) \otimes M\right) \\
= & \left(B \otimes \varphi_{M}\right) \circ\left(c_{H, B} \otimes M\right) \circ\left(\left(\left(\mu_{H} \otimes B\right) \circ(H \otimes \sigma)\right) \otimes M\right) \\
= & \left(B \otimes \varphi_{M}\right) \circ\left(c_{H, B} \otimes M\right) \circ\left(H \otimes \rho_{M}\right) .
\end{aligned}
$$

In particular, if $H=B, \varphi_{H}=\mu_{H}$ and $\sigma: K \rightarrow H \otimes H$ is a skew copairing between $H$ and H over K such that

$$
\left(\mu_{H} \otimes H\right) \circ(H \otimes \sigma)=\left(\left(\mu_{H} \circ c_{H, H}\right) \otimes H\right) \circ(H \otimes \sigma)
$$

we obtain that $\left(H, \mu_{H}, \rho_{H}=\left(H \otimes \mu_{H}\right) \circ\left(\left(c_{H, H} \circ \sigma\right) \otimes H\right)\right)$ is in ${ }_{H}^{H}$ Long. Moreover, if $\lambda_{H}$ is an isomorphism, the triple $\left(H, \mu_{H}, \rho_{H}\right)$ belongs to ${ }_{H}^{H}$ Long if and only if (48) holds.

Example 4. Let $H$ and $B$ be weak Hopf monoids in C. Let $\tau: H \otimes B \rightarrow K$ and $\sigma: K \rightarrow H \otimes B$ be morphisms in $C$. It is easy to show that $\left(B, \varphi_{B}=(B \otimes \tau) \circ\left(c_{H, B} \otimes B\right) \circ\left(H \otimes \delta_{B}\right)\right)$ is a left $H$-module if and only if (b1) and (b3) of Example 2 hold. Similarly, $\left(H, \rho_{H}=\left(B \otimes \mu_{H}\right) \circ\left(\left(c_{H, B} \circ\right.\right.\right.$ $\sigma) \otimes H)$ is a left $B$-comodule if and only if (c2) and (c3) of Example 3 hold. In any case, $\left(B, \varphi_{B}, \delta_{B}\right)$ and $\left(H, \mu_{H}, \rho_{H}\right)$ are objects in the category ${ }_{H}^{B}$ Long.

Remark 1. Please note that in the weak Hopf monoid setting, an object $M$ with the trivial morphisms $\varphi_{M}=\varepsilon_{H} \otimes M$ and $\rho_{M}=\eta_{B} \otimes M$ is not an object in ${ }_{H}^{B}$ Long because in this case neither $\left(M, \varphi_{M}\right)$ is a left $H$-module nor $\left(M, \rho_{M}\right)$ a left B-comodule.

Lemma 2. Let $H$ and $B$ be weak Hopf monoids and let $\left(M, \varphi_{M}, \rho_{M}\right)$ and $\left(N, \varphi_{N}, \rho_{N}\right)$ be in ${ }_{H}^{B}$ Long. Then, the idempotent morphisms $\nabla_{M \otimes N}$ and $\nabla_{M \otimes N^{\prime}}^{\prime}$ defined in (32) and (40) (for $\left.H=B\right)$, satisfy that

$$
\nabla_{M \otimes N}^{\prime} \circ \nabla_{M \otimes N}=\nabla_{M \otimes N} \circ \nabla_{M \otimes N}^{\prime}
$$

As a consequence, the morphism

$$
\Omega_{M \otimes N}=\nabla_{M \otimes N}^{\prime} \circ \nabla_{M \otimes N}
$$

is idempotent and there exist two morphisms $j_{M \otimes N}: M \times N \rightarrow M \otimes N$ and $q_{M \otimes N}: M \otimes N \rightarrow$ $M \times N$ such that $q_{M \otimes N} \circ j_{M \otimes N}=i d_{M \times N}$ and $j_{M \otimes N} \circ q_{M \otimes N}=\Omega_{M \otimes N}$ where $M \times N$ is the image of $\Omega_{M \otimes N}$.

Moreover, the following identities hold:

$$
\begin{array}{lll}
\nabla_{M \otimes N} \circ \Omega_{M \otimes N}=\Omega_{M \otimes N}, & q_{M \otimes N} \circ \nabla_{M \otimes N} \circ \Omega_{M \otimes N}=q_{M \otimes N}, & \nabla_{M \otimes N} \circ j_{M \otimes N}=j_{M \otimes N}, \\
\nabla_{M \otimes N}^{\prime} \circ \Omega_{M \otimes N}=\Omega_{M \otimes N}, & q_{M \otimes N} \circ \nabla_{M \otimes N}^{\prime} \circ \Omega_{M \otimes N}=q_{M \otimes N}, & \nabla_{M \otimes N}^{\prime} \circ j_{M \otimes N}=j_{M \otimes N}, \\
\Omega_{M \otimes N} \circ \nabla_{M \otimes N}=\Omega_{M \otimes N}, & \Omega_{M \otimes N} \circ \nabla_{M \otimes N} \circ j_{M \otimes N}=j_{M \otimes N}, & q_{M \otimes N} \circ \nabla_{M \otimes N}=q_{M \otimes N}, \\
\Omega_{M \otimes N} \circ \nabla_{M \otimes N}^{\prime}=\Omega_{M \otimes N}, & \Omega_{M \otimes N} \circ \nabla_{M \otimes N}^{\prime} \circ j_{M \otimes N}=j_{M \otimes N}, & q_{M \otimes N} \circ \nabla_{M \otimes N}^{\prime}=q_{M \otimes N} .
\end{array}
$$

Proof. Indeed, let $\left(M, \varphi_{M}, \rho_{M}\right)$ and $\left(N, \varphi_{N}, \rho_{N}\right)$ be in ${ }_{H}^{B}$ Long. Then,

$$
\begin{aligned}
& \nabla_{M \otimes N}^{\prime} \circ \nabla_{M \otimes N} \\
= & \left(\left(\varepsilon_{B} \circ \mu_{B}\right) \otimes M \otimes N\right) \circ\left(B \otimes c_{M, B} \otimes N\right) \\
& \circ\left(\left(\left(B \otimes \varphi_{M}\right) \circ\left(c_{H, B} \otimes M\right) \circ\left(H \otimes \rho_{M}\right)\right) \otimes\left(\left(B \otimes \varphi_{N}\right) \circ\left(c_{H, B} \otimes N\right) \circ\left(H \otimes \rho_{N}\right)\right)\right) \\
& \circ\left(H \otimes c_{H, M} \otimes N\right) \circ\left(\left(\delta_{H} \circ \eta_{H}\right) \otimes M \otimes N\right)(\text { by (44)) }
\end{aligned}
$$


$=\nabla_{M \otimes N} \circ \nabla_{M \otimes N}^{\prime}$ (by the naturality of $c$ ).

Finally, (51)-(54) follows easily from (49) and the properties of $j_{M \otimes N}$ and $q_{M \otimes N}$.

Lemma 3. Let $H$ and $B$ be weak Hopf monoids and let $\left(M, \varphi_{M}, \rho_{M}\right)$ and $\left(N, \varphi_{N}, \rho_{N}\right)$ be in ${ }_{H}^{B}$ Long. Then, the idempotent morphisms $\nabla_{M \otimes N}, \nabla_{M \otimes N}^{\prime}$ and $\Omega_{M \otimes N}$, defined in (32), (40) and (50), satisfy that

$$
\begin{gathered}
\varphi_{M \otimes N} \circ\left(H \otimes \nabla_{M \otimes N}^{\prime}\right)=\nabla_{M \otimes N}^{\prime} \circ \varphi_{M \otimes N}, \\
\left(B \otimes \nabla_{M \otimes N}\right) \circ \rho_{M \otimes N}=\rho_{M \otimes N} \circ \nabla_{M \otimes N}, \\
\varphi_{M \otimes N} \circ\left(H \otimes \Omega_{M \otimes N}\right)=\Omega_{M \otimes N} \circ \varphi_{M \otimes N}, \\
\left(B \otimes \Omega_{M \otimes N}\right) \circ \rho_{M \otimes N}=\rho_{M \otimes N} \circ \Omega_{M \otimes N},
\end{gathered}
$$

where $\varphi_{M \otimes N}$ and $\rho_{M \otimes N}$ are the morphisms defined in (30) and (38) respectively.

Proof. Let $\left(M, \varphi_{M}, \rho_{M}\right)$ and $\left(N, \varphi_{N}, \rho_{N}\right)$ be in ${ }_{H}^{B}$ Long. First we have that

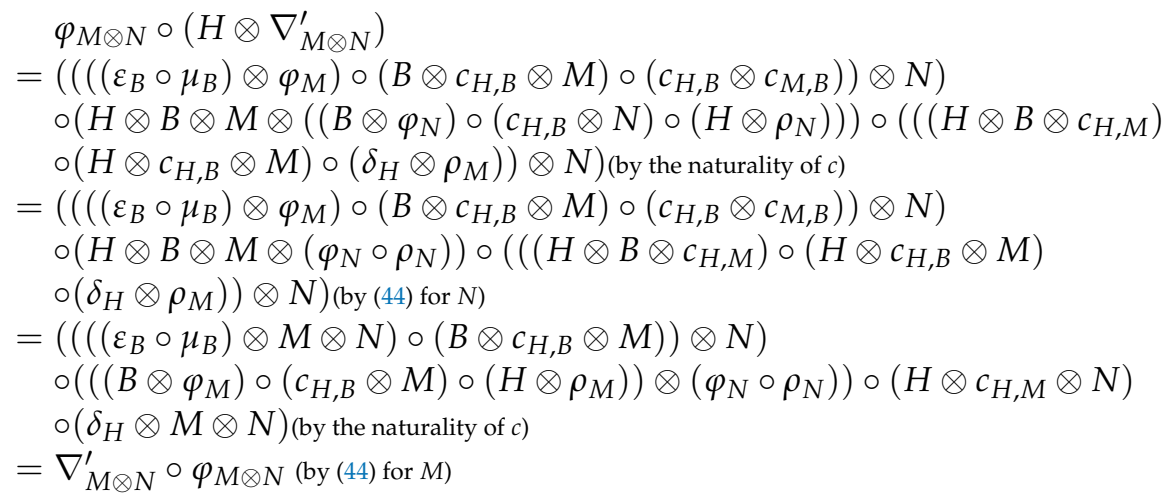
and (55) holds. Secondly,

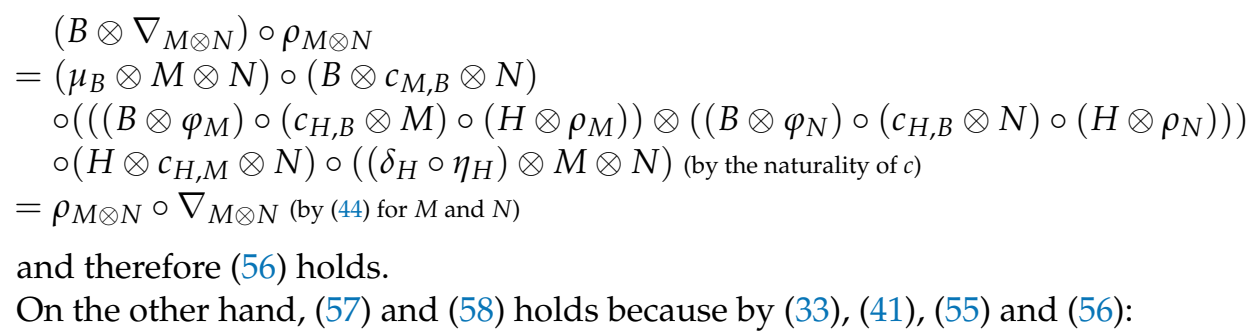

$$
\begin{gathered}
\Omega_{M \otimes N} \circ \varphi_{M \otimes N}=\nabla_{M \otimes N}^{\prime} \circ \varphi_{M \otimes N}=\varphi_{M \otimes N} \circ\left(H \otimes \nabla_{M \otimes N}^{\prime}\right)=\varphi_{M \otimes N} \circ\left(H \otimes \Omega_{M \otimes N}\right), \\
\rho_{M \otimes N} \circ \Omega_{M \otimes N}=\rho_{M \otimes N} \circ \nabla_{M \otimes N}=\left(B \otimes \nabla_{M \otimes N}\right) \circ \rho_{M \otimes N}=\left(B \otimes \Omega_{M \otimes N}\right) \circ \rho_{M \otimes N} .
\end{gathered}
$$

Lemma 4. Let $H$ and $B$ be weak Hopf monoids and let $\left(M, \varphi_{M}, \rho_{M}\right),\left(N, \varphi_{N}, \rho_{N}\right)$ and $\left(P, \varphi_{P}, \rho_{P}\right)$ be in ${ }_{H}^{B}$ Long. Then, the idempotent morphisms $\nabla_{M \otimes N}, \nabla_{M \otimes N}^{\prime}$ and $\Omega_{M \otimes N}$, defined in (32), (40) and (50), satisfy that

$$
\begin{aligned}
& \left(M \otimes \nabla_{N \otimes P}^{\prime}\right) \circ\left(\nabla_{M \otimes N} \otimes P\right)=\left(\nabla_{M \otimes N} \otimes P\right) \circ\left(M \otimes \nabla_{N \otimes P}^{\prime}\right), \\
& \left(M \otimes \nabla_{N \otimes P}\right) \circ\left(\nabla_{M \otimes N}^{\prime} \otimes P\right)=\left(\nabla_{M \otimes N}^{\prime} \otimes P\right) \circ\left(M \otimes \nabla_{N \otimes P}\right), \\
& \left(M \otimes \Omega_{N \otimes P}\right) \circ\left(\Omega_{M \otimes N} \otimes P\right)=\left(\Omega_{M \otimes N} \otimes P\right) \circ\left(M \otimes \Omega_{N \otimes P}\right), \\
& \left(M \otimes \nabla_{N \otimes P}\right) \circ\left(\Omega_{M \otimes N} \otimes P\right)=\left(\Omega_{M \otimes N} \otimes P\right) \circ\left(M \otimes \nabla_{N \otimes P}\right), \\
& \left(M \otimes \Omega_{N \otimes P}\right) \circ\left(\nabla_{M \otimes N} \otimes P\right)=\left(\nabla_{M \otimes N} \otimes P\right) \circ\left(M \otimes \Omega_{N \otimes P}\right), \\
& \left(M \otimes \nabla_{N \otimes P}^{\prime}\right) \circ\left(\Omega_{M \otimes N} \otimes P\right)=\left(\Omega_{M \otimes N} \otimes P\right) \circ\left(M \otimes \nabla_{N \otimes P}^{\prime}\right),
\end{aligned}
$$




$$
\left(M \otimes \Omega_{N \otimes P}\right) \circ\left(\nabla_{M \otimes N}^{\prime} \otimes P\right)=\left(\nabla_{M \otimes N}^{\prime} \otimes P\right) \circ\left(M \otimes \Omega_{N \otimes P}\right) .
$$

Proof. The proofs for (61)-(65) follow directly from (59) and (60). The proof for (60) is similar to the one of (59). Then, we only need to show that (59) holds. Indeed:

$$
\begin{aligned}
& \left(M \otimes \nabla_{N \otimes P}^{\prime}\right) \circ\left(\nabla_{M \otimes N} \otimes P\right) \\
= & \left(\varphi_{M} \otimes\left(\varepsilon_{B} \circ \mu_{B}\right) \otimes N \otimes P\right) \circ\left(H \otimes M \otimes B \otimes c_{N, B} \otimes P\right) \\
& \circ\left(H \otimes M \otimes\left(\left(B \otimes \varphi_{N}\right) \circ\left(c_{H, B} \otimes N\right) \circ\left(H \otimes \rho_{N}\right)\right) \otimes \rho_{P}\right) \\
& \circ\left(\left(\left(H \otimes c_{H, M}\right) \circ\left(\left(\delta_{H} \circ \eta_{H}\right) \otimes M\right)\right) \otimes N \otimes P\right) \text { (by (44)) } \\
= & \left.\left(\nabla_{M \otimes N} \otimes P\right) \circ\left(M \otimes \nabla_{N \otimes P}^{\prime}\right) \text { (by the naturality of } c\right) .
\end{aligned}
$$

Now we will define a tensor product in the categories of Long dimodules. The proof follows a similar pattern for each side. Then, we only get the computations for the leftleft side.

Proposition 1. Let $H$ and $B$ be weak Hopf monoids and let $\left(M, \varphi_{M}, \rho_{M}\right)$ and $\left(N, \varphi_{N}, \rho_{N}\right)$ be in ${ }_{H}^{B}$ Long. Then, the image of the idempotent morphism $\Omega_{M \otimes N}$, defined in (50), belongs to ${ }_{H}^{B}$ Long with $\mathrm{H}$-module and B-comodule structures

$$
\varphi_{M \times N}=q_{M \otimes N} \circ \varphi_{M \otimes N} \circ\left(H \otimes j_{M \otimes N}\right)
$$

and

$$
\rho_{M \times N}=\left(B \otimes q_{M \otimes N}\right) \circ \rho_{M \otimes N} \circ j_{M \otimes N},
$$

respectively. Moreover, if $f:\left(M, \varphi_{M}, \rho_{M}\right) \rightarrow\left(M^{\prime}, \varphi_{M^{\prime}}, \rho_{M^{\prime}}\right), g:\left(N, \varphi_{N}, \rho_{N}\right) \rightarrow\left(N^{\prime}, \varphi_{N^{\prime}}, \rho_{N^{\prime}}\right)$ are morphisms in ${ }_{H}^{B}$ Long, then,

$$
f \times g=q_{M^{\prime} \times N^{\prime}} \circ(f \otimes g) \circ j_{M \times N}: M \times N \rightarrow M^{\prime} \times N^{\prime}
$$

is a morphism in ${ }_{H}^{B}$ Long between $\left(M \times N, \varphi_{M \times N}, \rho_{M \times N}\right)$ and $\left(M^{\prime} \times N^{\prime}, \varphi_{M^{\prime} \times N^{\prime}}, \rho_{M^{\prime} \times N^{\prime}}\right)$.

Proof. Let $H$ and $B$ be weak Hopf monoids and let $\left(M, \varphi_{M}, \rho_{M}\right)$ and $\left(N, \varphi_{N}, \rho_{N}\right)$ be in ${ }_{H}^{B}$ Long. Let $M \times N$ the image of the idempotent morphism $\Omega_{M \otimes N}$. Define the action $\varphi_{M \times N}$ by

$$
\varphi_{M \times N}=q_{M \otimes N} \circ \varphi_{M \otimes N} \circ\left(H \otimes j_{M \otimes N}\right)
$$

and the coaction $\rho_{M \times N}$ by

$$
\rho_{M \times N}=\left(B \otimes q_{M \otimes N}\right) \circ \rho_{M \otimes N} \circ j_{M \otimes N} .
$$

The pair $\left(M \otimes N, \varphi_{M \times N}\right)$ is a left $H$-module because

$$
\varphi_{M \times N} \circ\left(\eta_{H} \otimes M \times N\right)=q_{M \otimes N} \circ \nabla_{M \otimes N} \circ j_{M \otimes N} \stackrel{(53)}{=} q_{M \otimes N} \circ j_{M \otimes N}=i d_{M \times N}
$$

and

$\varphi_{M \times N} \circ\left(H \otimes \varphi_{M \times N}\right) \stackrel{(57)}{=} q_{M \otimes N} \circ \varphi_{M \otimes N} \circ\left(H \otimes\left(\varphi_{M \otimes N} \circ\left(H \otimes j_{M \otimes N}\right)\right)\right) \stackrel{(31)}{=} \varphi_{M \times N} \circ\left(\mu_{H} \otimes M \times N\right)$.

Similarly, by (54) we obtain that $\left(\varepsilon_{B} \otimes M \otimes N\right) \circ \rho_{M \times N}=i d_{M \times N}$ and, by (39) and (58), we have that

$$
\left(\rho_{M \times N} \otimes M \otimes N\right) \circ \rho_{M \times N}=\left(\delta_{B} \otimes M \otimes N\right) \circ \rho_{M \times N} .
$$

Therefore, $\left(M \otimes N, \rho_{M \times N}\right)$ is a left $B$-comodule.

Also, $\left(M \otimes N, \varphi_{M \times N}, \rho_{M \times N}\right)$ is an object in ${ }_{H}^{B}$ Long because

$$
\begin{aligned}
& \left(B \otimes \varphi_{M \times N}\right) \circ\left(c_{H, B} \otimes M \times N\right) \circ\left(H \otimes \rho_{M \times N}\right) \\
= & \left(B \otimes\left(q_{M \otimes N} \circ \varphi_{M \otimes N}\right)\right) \circ\left(c_{H, B} \otimes M \otimes N\right) \circ\left(H \otimes\left(\rho_{M \otimes N} \circ j_{M \otimes N}\right)\right) \text { (by (57)) } \\
= & \left(\mu_{B} \otimes q_{M \otimes N}\right) \circ\left(B \otimes c_{M, B} \otimes N\right)
\end{aligned}
$$




$$
\begin{aligned}
& \circ\left(\left(\left(B \otimes \varphi_{M}\right) \circ\left(c_{H, B} \otimes M\right) \circ\left(H \otimes \rho_{M}\right)\right) \otimes\left(\left(B \otimes \varphi_{N}\right) \circ\left(c_{H, B} \otimes N\right) \circ\left(H \otimes \rho_{N}\right)\right)\right) \\
& \left.\circ\left(H \otimes c_{H, M} \otimes N\right) \circ\left(\delta_{H} \otimes j_{M \otimes N}\right) \text { (by the naturality of } c\right) \\
= & \left(\mu_{B} \otimes q_{M \otimes N}\right) \circ\left(B \otimes c_{M, B} \otimes N\right) \circ\left(\left(\rho_{M} \circ \varphi_{M}\right) \otimes\left(\rho_{N} \circ \varphi_{N}\right)\right) \circ\left(H \otimes c_{H, M} \otimes N\right) \\
& \left.\circ\left(\delta_{H} \otimes j_{M \otimes N}\right) \text { (by (44) for } M \text { and } N\right) \\
= & \rho_{M \times N} \circ \varphi_{M \times N} \text { (by (57)) }
\end{aligned}
$$

On the other hand, if $f:\left(M, \varphi_{M}, \rho_{M}\right) \rightarrow\left(M^{\prime}, \varphi_{M^{\prime}}, \rho_{M^{\prime}}\right)$ and $g:\left(N, \varphi_{N}, \rho_{N}\right) \rightarrow$ $\left(N^{\prime}, \varphi_{N^{\prime}}, \rho_{N^{\prime}}\right)$ are morphisms in ${ }_{H}^{B}$ Long, by (35) and (43), we have that

$$
(f \otimes g) \circ \Omega_{M \otimes N}=\Omega_{M^{\prime} \otimes N^{\prime}} \circ(f \otimes g)
$$

holds. Define $f \times g: M \times N \rightarrow M^{\prime} \times N^{\prime}$ as $f \times g=q_{M^{\prime} \times N^{\prime}} \circ(f \otimes g) \circ j_{M \times N}$. Then, $f \times g$ is a morphism in ${ }_{H}^{B}$ Long because

$$
\begin{aligned}
& \varphi_{M^{\prime} \times N^{\prime}} \circ(H \otimes(f \times g)) \\
= & \left.q_{M^{\prime} \otimes N^{\prime}} \circ \varphi_{M^{\prime} \otimes N^{\prime}} \circ\left(H \otimes\left(\Omega_{M^{\prime} \times N^{\prime}} \circ(f \otimes g) \circ j_{M \otimes N}\right)\right) \text { (by definition of } f \times g\right) \\
= & q_{M^{\prime} \otimes N^{\prime}} \circ \varphi_{M^{\prime} \otimes N^{\prime}} \circ\left(H \otimes\left((f \otimes g) \circ j_{M \otimes N}\right)\right)(\text { by (66)) } \\
= & q_{M^{\prime} \otimes N^{\prime}} \circ(f \otimes g) \circ \varphi_{M \times N} \circ\left(H \otimes j_{M \otimes N}\right) \text { (by the condition of morphism of left } H \text {-modules for } f \text { and } g \text { ) } \\
= & (f \times g) \circ \varphi_{M \times N} \text { (by (57)) }
\end{aligned}
$$

and

$$
\begin{aligned}
& \rho_{M^{\prime} \times N^{\prime}} \circ(f \times g) \\
= & \left(B \otimes q_{M^{\prime} \otimes N^{\prime}}\right) \circ \rho_{M^{\prime} \otimes N^{\prime}} \circ \Omega_{M^{\prime} \times N^{\prime}} \circ(f \otimes g) \circ j_{M \otimes N} \text { (by definition) } \\
= & \left(B \otimes q_{M^{\prime} \otimes N^{\prime}}\right) \circ \rho_{M^{\prime} \otimes N^{\prime}} \circ(f \otimes g) \circ j_{M \otimes N} \text { (by (66)) } \\
= & \left(B \otimes\left(q_{M^{\prime} \otimes N^{\prime}} \circ(f \otimes g)\right)\right) \circ \rho_{M \otimes N} \circ j_{M \otimes N} \text { (by the condition of morphism of left } B \text {-comodules for } f \\
& \text { and } g) \\
= & (B \otimes(f \times g)) \circ \rho_{M \times N} \text { (by (58)). }
\end{aligned}
$$

Therefore, the proof is complete.

Lemma 5. Let $H$ and $B$ be weak Hopf monoids and let $\left(M, \varphi_{M}, \rho_{M}\right),\left(N, \varphi_{N}, \rho_{N}\right)$ and $\left(P, \varphi_{P}, \rho_{P}\right)$ be in ${ }_{H}^{B}$ Long. Then, the following equalities hold:

$$
\begin{gathered}
\left(M \otimes j_{N \otimes P}\right) \circ \Omega_{M \otimes(N \times P)} \circ\left(M \otimes q_{N \otimes P}\right)=\left(j_{M \otimes N} \otimes P\right) \circ \Omega_{(M \times N) \otimes P} \circ\left(q_{M \otimes N} \otimes P\right), \\
\left(M \otimes j_{N \otimes P}\right) \circ \Omega_{M \otimes(N \times P)} \circ\left(M \otimes q_{N \otimes P}\right)=\left(\Omega_{M \otimes N} \otimes P\right) \circ\left(M \otimes \Omega_{N \otimes P}\right) \\
\Omega_{(M \times N) \otimes P}=\left(q_{M \otimes N} \otimes P\right) \circ\left(M \otimes \Omega_{N \otimes P}\right) \circ\left(j_{M \otimes N} \otimes P\right) \\
\Omega_{M \otimes(N \times P)}=\left(M \otimes q_{N \otimes P}\right) \circ\left(\Omega_{M \otimes N} \otimes P\right) \circ\left(M \otimes j_{N \otimes P}\right) .
\end{gathered}
$$

Proof. The proof for the identity (67) is:

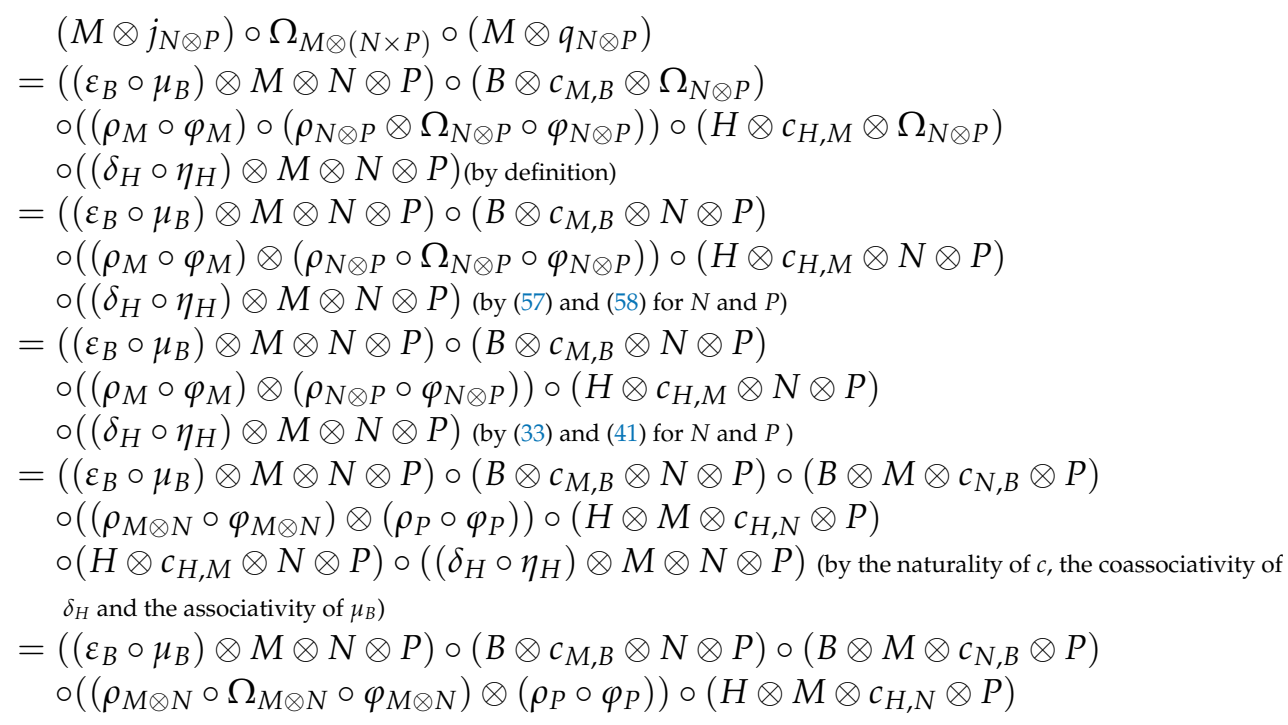


$\circ\left(H \otimes c_{H, M} \otimes N \otimes P\right) \circ\left(\left(\delta_{H} \circ \eta_{H}\right) \otimes M \otimes N \otimes P\right)$ (by (33) and (41) for $M$ and $N$ )

$=\left(\left(\varepsilon_{B} \circ \mu_{B}\right) \otimes \Omega_{M \otimes N} \otimes P\right) \circ\left(B \otimes c_{M, B} \otimes N \otimes P\right) \circ\left(B \otimes M \otimes c_{N, B} \otimes P\right)$

$\circ\left(\left(\rho_{M \otimes N} \circ \Omega_{M \otimes N} \circ \varphi_{M \otimes N}\right) \otimes\left(\rho_{P} \circ \varphi_{P}\right)\right) \circ\left(H \otimes M \otimes c_{H, N} \otimes P\right)$

$\circ\left(H \otimes c_{H, M} \otimes N \otimes P\right) \circ\left(\left(\delta_{H} \circ \eta_{H}\right) \otimes \Omega_{M \otimes N} \otimes P\right)$ (by (57) and (58) for $M$ and $N$ )

$=\left(j_{M \otimes N} \otimes P\right) \circ \Omega_{(M \times N) \otimes P} \circ\left(q_{M \otimes N} \otimes P\right)$ (by definition).

On the other hand, (68) follows by

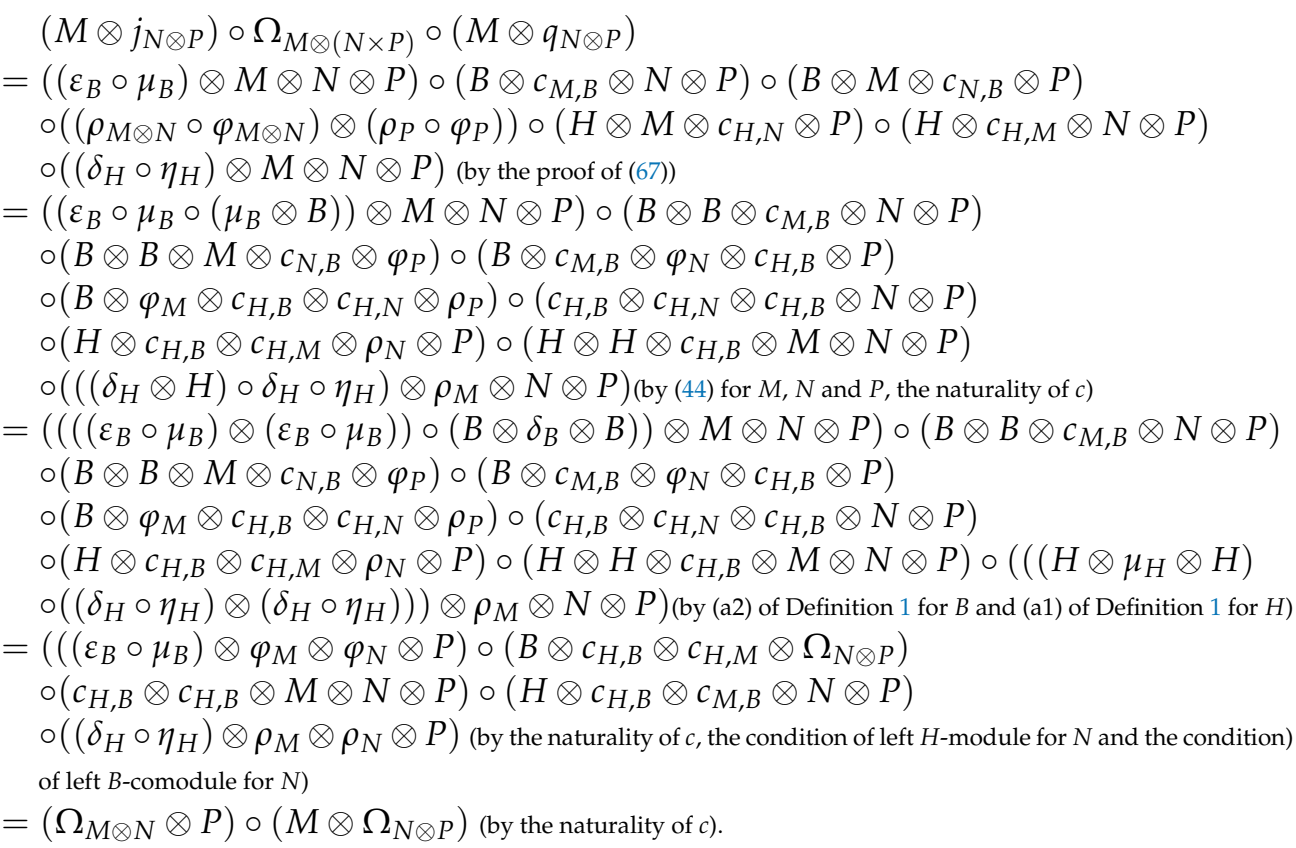

Finally, note that by (61), (67) and (68)

$$
\begin{gathered}
\left(j_{M \otimes N} \otimes P\right) \circ \Omega_{(M \times N) \otimes P} \circ\left(q_{M \otimes N} \otimes P\right)=\left(\Omega_{M \otimes N} \otimes P\right) \circ\left(M \otimes \Omega_{N \otimes P}\right) \circ\left(\Omega_{M \otimes N} \otimes P\right) \\
=\left(\left(j_{M \otimes N} \circ q_{M \otimes N}\right) \otimes P\right) \circ\left(M \otimes \Omega_{N \otimes P}\right) \circ\left(\left(j_{M \otimes N} \circ q_{M \otimes N}\right) \otimes P\right) .
\end{gathered}
$$

Therefore, (69) holds because $j_{M \otimes N} \otimes P$ is a monomorphism and $q_{M \otimes N} \otimes P$ is an epimorphism. The proof for (70) is similar and we leave the details to the reader.

Proposition 2. Let $H$ and $B$ be weak Hopf monoids and let $\left(M, \varphi_{M}, \rho_{M}\right),\left(N, \varphi_{N}, \rho_{N}\right)$ and $\left(P, \varphi_{P}, \rho_{P}\right)$ be in ${ }_{H}^{B}$ Long. Then, the morphism

$$
a_{M, N, P}:(M \times N) \times P \rightarrow M \times(N \times P),
$$

defined by

$$
a_{M, N, P}=q_{M \otimes(N \times P)} \circ\left(M \otimes q_{N \otimes P}\right) \circ\left(j_{M \otimes N} \otimes P\right) \circ j_{(M \times N) \otimes P}
$$

is a natural isomorphism in ${ }_{H}^{B}$ Long and satisfies the Pentagon Axiom.

Proof. First, note that the naturality of $a$ follows from (66). Secondly, by (68), it is easy to show that the inverse of $a_{M, N, P}$ is

$$
a_{M, N, P}^{-1}=q_{(M \times N) \otimes P} \circ\left(q_{M \otimes N} \otimes P\right) \circ\left(M \otimes j_{N \otimes P}\right) \circ j_{M \otimes(N \times P)} .
$$

On the other hand, $a_{M, N, P}$ is a morphism in ${ }_{H}^{B}$ Long because we have

$$
\begin{aligned}
& \varphi_{M \times(N \times P)} \circ\left(H \otimes a_{M, N, P}\right) \\
= & q_{M \otimes(N \times P)} \circ\left(\varphi_{M} \otimes\left(q_{N \times P} \circ \varphi_{N \otimes P}\right)\right) \circ\left(H \otimes c_{H, M} \otimes N \otimes P\right) \circ\left(H \otimes H \otimes \left(\left(M \otimes j_{N \otimes P}\right)\right.\right. \\
& \left.\left.\circ \Omega_{M \otimes(N \times P)} \circ\left(M \otimes q_{N \otimes P}\right)\right)\right) \circ\left(\delta_{H} \otimes\left(\left(j_{M \otimes N} \otimes P\right) \circ j_{(M \times N) \otimes P}\right)\right) \text { (by definition) } \\
= & q_{M \otimes(N \times P)} \circ\left(\varphi_{M} \otimes\left(q_{N \times P} \circ \varphi_{N \otimes P}\right)\right) \circ\left(H \otimes c_{H, M} \otimes \Omega_{N \otimes P}\right) \circ\left(\delta _ { H } \otimes \left(\left(j_{M \otimes N} \otimes P\right)\right.\right.
\end{aligned}
$$




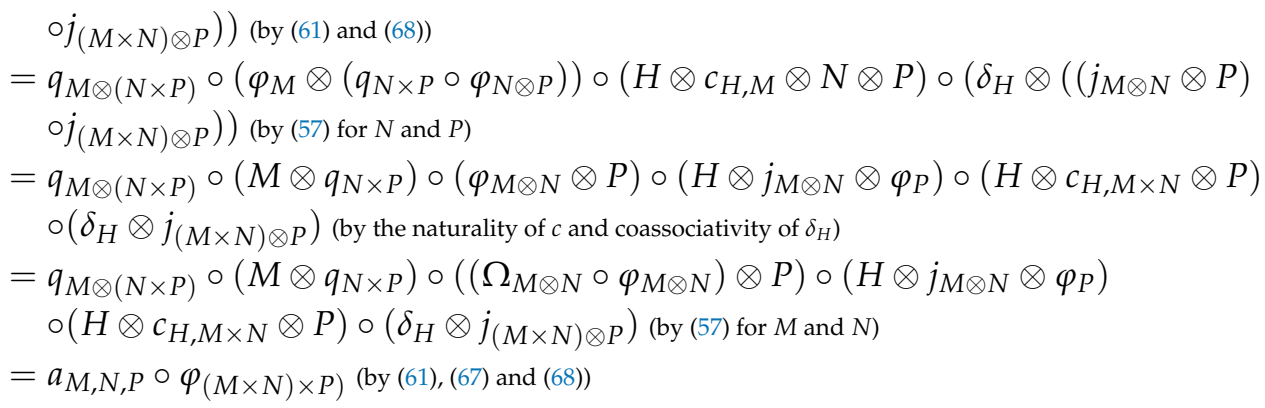

and

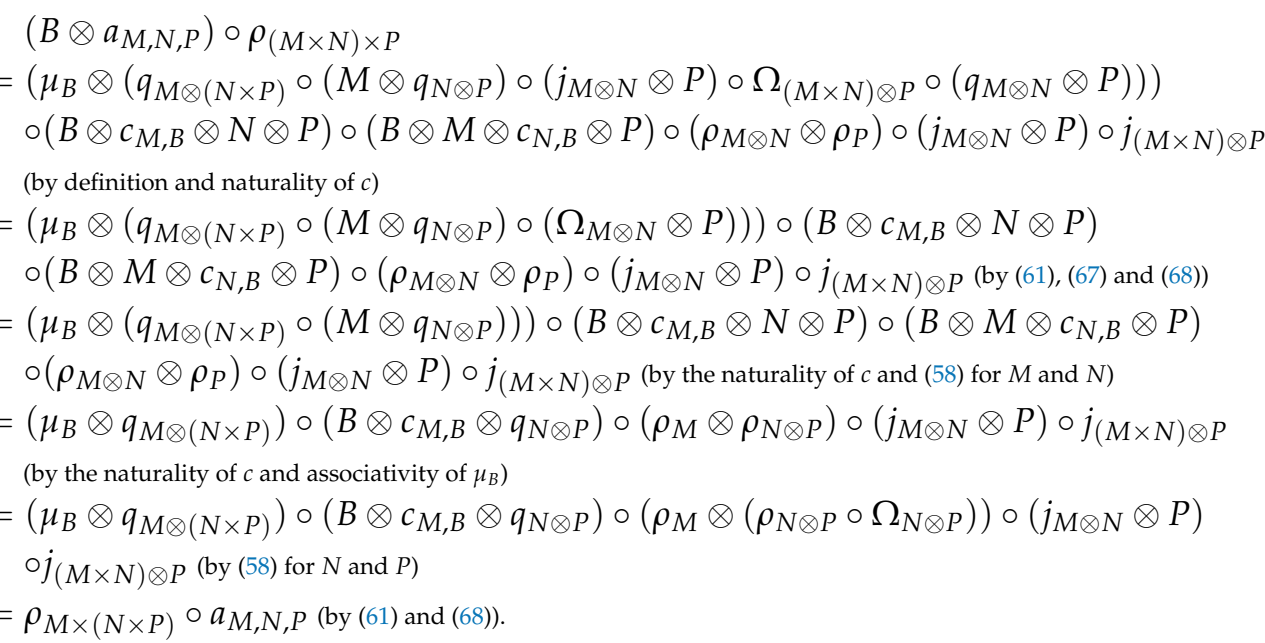

Then, consequently, the Pentagon Axiom holds because, if $\left(M, \varphi_{M}, \rho_{M}\right),\left(N, \varphi_{N}, \rho_{N}\right)$, $\left(P, \varphi_{P}, \rho_{P}\right)$ and $\left(Q, \varphi_{Q}, \rho_{Q}\right)$ are in ${ }_{H}^{B}$ Long,

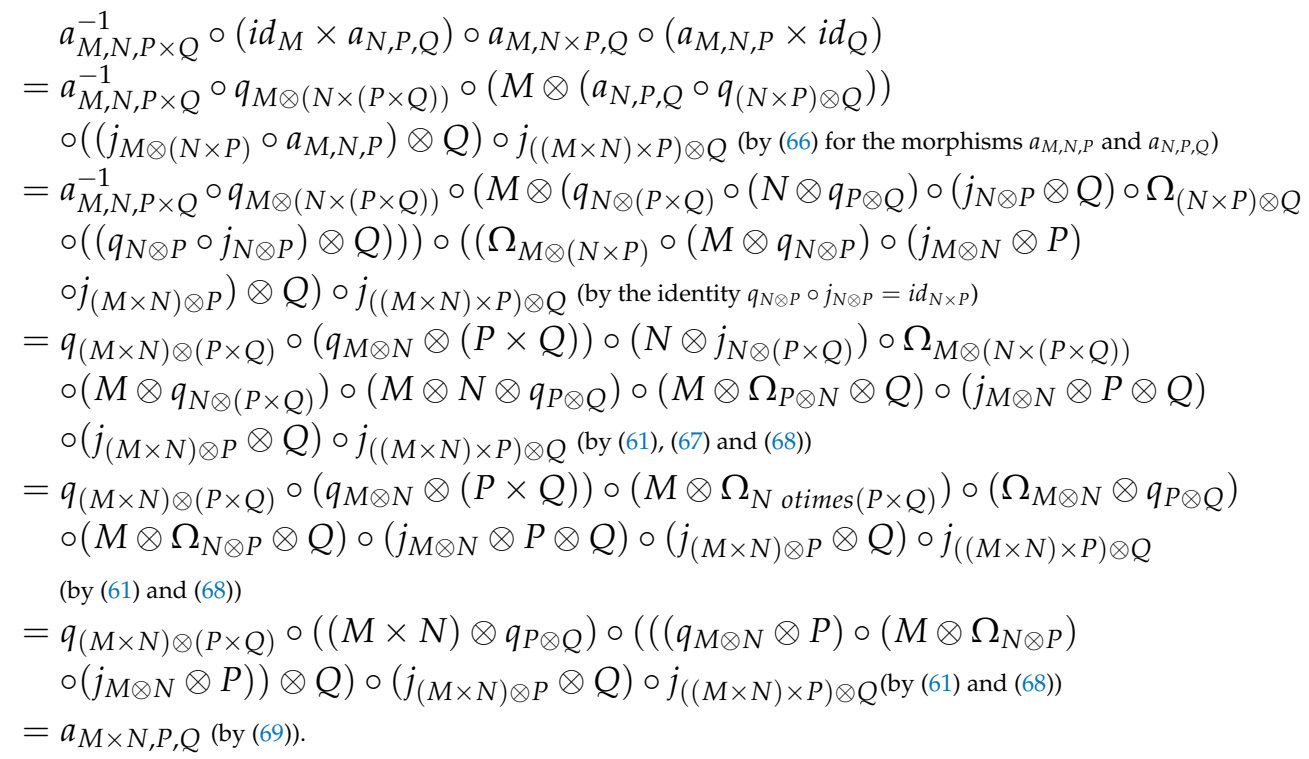

Lemma 6. Let $H$ and $B$ be weak Hopf monoids and let $\left(M, \varphi_{M}, \rho_{M}\right)$ be in ${ }_{H}^{B}$ Long. The following identities hold:

$$
\begin{gathered}
\Omega_{\left(H_{L} \otimes B_{L}\right) \otimes M}=\left(p_{H}^{L} \otimes\left(p_{B}^{L} \circ \mu_{B}\right) \otimes M\right) \circ\left(H \otimes B \otimes\left(\rho_{M} \circ \varphi_{M}\right)\right) \circ\left(\left(\left(H \otimes c_{H, B}\right) \circ\left(\left(\delta_{H} \circ i_{H}^{L}\right) \otimes i_{B}^{L}\right)\right) \otimes M\right), \\
\Omega_{\left(H_{L} \otimes B_{L}\right) \otimes M} \circ\left(H_{L} \otimes\left(\left(p_{B}^{L} \otimes M\right) \circ \rho_{M}\right)=\left(p_{H}^{L} \otimes p_{B}^{L} \otimes M\right) \circ\left(H \otimes\left(\rho_{M} \circ \varphi_{M}\right)\right) \circ\left(\left(\delta_{H} \circ i_{H}^{L}\right) \otimes M\right),\right. \\
\Omega_{M \otimes\left(H_{L} \otimes B_{L}\right)}
\end{gathered}
$$


$=\left(M \otimes p_{H}^{L} \otimes\left(p_{B}^{L} \circ \mu_{B} \circ\left(\bar{\Pi}_{B}^{R} \otimes i_{B}^{L}\right)\right)\right) \circ\left(\left(\left(M \otimes c_{B, H}\right) \circ\left(c_{B, M} \otimes H\right) \circ\left(B \otimes\left(\varphi_{M} \circ c_{M, H}\right) \otimes H\right)\right.\right.$

$$
\begin{gathered}
\left.\left.\circ\left(\rho_{M} \otimes\left(\left(\bar{\Pi}_{B}^{L} \otimes H\right) \circ \delta_{H} \circ i_{H}^{L}\right)\right)\right) \otimes B_{L}\right), \\
\Omega_{M \otimes\left(H_{L} \otimes B_{L}\right)} \circ\left(\varphi_{M} \otimes p_{H}^{L} \otimes p_{B}^{L}\right)
\end{gathered}
$$

$=\left(\varphi_{M} \otimes\left(p_{H}^{L} \circ \mu_{H}\right) \otimes\left(p_{B}^{L} \circ \mu_{B} \circ\left(\bar{\Pi}_{B}^{R} \otimes B\right)\right)\right) \circ\left(\left(\left(H \otimes c_{H, M} \otimes c_{B, H}\right) \circ\left(\left(\left(\left(H \otimes \Pi_{H}^{L}\right) \circ \delta_{H}\right) \otimes\left(c_{B, M} \circ \rho_{M}\right) \otimes H\right) \otimes B\right)\right.\right.$.

Proof. The proof for (71) is the following:

$\Omega_{\left(H_{L} \otimes B_{L}\right) \otimes M}$

$=\left(\left(p_{H}^{L} \otimes\left(\left(\left(\varepsilon_{B} \circ \mu_{B}\right) \otimes p_{B}^{L}\right) \circ\left(B \otimes c_{B, B}\right) \circ\left(\delta_{B} \otimes B\right)\right)\right) \otimes M\right) \circ\left(H \otimes B \otimes\left(\rho_{M} \circ \varphi_{M}\right)\right)$

$\circ\left(H \otimes c_{H, B} \otimes M\right) \circ\left(\left(\left(\mu_{H} \otimes H\right) \circ\left(H \otimes c_{H, H}\right) \circ\left(\left(\delta_{H} \circ \eta_{H}\right) \otimes i_{H}^{L}\right)\right) \otimes i_{B}^{L} \otimes M\right)$ (by the naturality of $c$ )

$=\left(p_{H}^{L} \otimes\left(p_{B}^{L} \circ \mu_{B} \circ\left(B \otimes \Pi_{B}^{L}\right)\right) \otimes M\right) \circ\left(H \otimes B \otimes\left(\rho_{M} \circ \varphi_{M}\right)\right) \circ\left(H \otimes c_{H, B} \otimes M\right)$ $\circ\left(\left(\left(H \otimes \Pi_{H}^{L}\right) \circ \delta_{H} \circ i_{H}^{L}\right) \otimes i_{B}^{L} \otimes M\right)$ (by (9) and (10))

$=\left(\left(p_{H}^{L} \otimes\left(p_{B}^{L} \circ \mu_{B}\right) \otimes M\right) \circ\left(H \otimes B \otimes\left(\rho_{M} \circ \varphi_{M}\right)\right)\right.$ $\circ\left(\left(\left(H \otimes c_{H, B}\right) \circ\left(\left(\delta_{H} \circ i_{H}^{L}\right) \otimes i_{B}^{L}\right)\right) \otimes M\right)($ by (5) and (7)).

As a consequence of (71), we have

$\Omega_{\left(H_{L} \otimes B_{L}\right) \otimes M} \circ\left(H_{L} \otimes\left(\left(p_{B}^{L} \otimes M\right) \circ \rho_{M}\right)\right.$

$=\left(p_{H}^{L} \otimes p_{B}^{L} \otimes \varphi_{M}\right) \circ\left(H \otimes c_{H, B} \otimes M\right) \circ\left(\left(\delta_{H} \circ i_{H}^{L}\right) \otimes\left(\left(\left(\Pi_{B}^{L} * i d_{B}\right) \otimes M\right) \circ \rho_{M}\right)\right)$ (by the naturality of $c$ )

$=\left(\left(p_{H}^{L} \otimes p_{B}^{L} \otimes M\right) \circ\left(H \otimes\left(\rho_{M} \circ \varphi_{M}\right)\right) \circ\left(\left(\delta_{H} \circ i_{H}^{L}\right) \otimes M\right)\right.$ (by (25) and (44))

and (72) holds.

On the other hand, (73) follows by

$\Omega_{M \otimes\left(H_{L} \otimes B_{L}\right)}$

$=\left(\left(\varepsilon_{B} \circ \mu_{B}\right) \otimes \varphi_{M} \otimes p_{H}^{L} \otimes p_{B}^{L}\right) \circ\left(B \otimes\left(\left(c_{H, B} \otimes M \otimes H\right) \circ\left(H \otimes c_{M, B} \otimes H\right)\right.\right.$ $\left.\left.\circ\left(c_{M, H} \otimes c_{H, B}\right)\right) \otimes B\right) \circ\left(\rho_{M} \otimes\left(\left(H \otimes \mu_{H}\right) \circ\left(\left(\delta_{H} \circ \eta_{H}\right) \otimes i_{H}^{L}\right)\right) \otimes\left(\delta_{B} \circ i_{B}^{L}\right)\right)$ (by the naturality of $c$ and (44))

$=\left(\left(\varepsilon_{B} \circ \mu_{B}\right) \otimes \varphi_{M} \otimes p_{H}^{L} \otimes p_{B}^{L}\right) \circ\left(B \otimes\left(\left(c_{H, B} \otimes M \otimes H\right) \circ\left(H \otimes c_{M, B} \otimes H\right)\right.\right.$ $\left.\left.\circ\left(c_{M, H} \otimes c_{H, B}\right)\right) \otimes B\right) \circ\left(\rho_{M} \otimes\left(\left(\left(\bar{\Pi}_{H}^{L} \otimes H\right) \circ \delta_{H} \circ i_{H}^{L}\right) \otimes\left(\delta_{B} \circ i_{B}^{L}\right)\right)\right.$ (by (15))

$=\left(M \otimes p_{H}^{L} \otimes\left(\left(\left(\varepsilon_{B} \circ \mu_{B}\right) \otimes p_{B}^{L}\right) \circ\left(B \otimes\left(\delta_{B} \circ i_{B}^{L}\right)\right)\right)\right) \circ\left(M \otimes c_{B, H} \otimes B_{L}\right)$ $\circ\left(c_{B, M} \otimes H \otimes B_{L}\right) \circ\left(B \otimes\left(\varphi_{M} \circ c_{M, H}\right) \otimes H \otimes B_{L}\right)$

$\circ\left(\rho_{M} \otimes\left(\left(\left(\bar{\Pi}_{H}^{L} \otimes H\right) \circ \delta_{H} \circ i_{H}^{L}\right) \otimes B_{L}\right)\right.$ (by the naturality of $\left.c\right)$

$=\left(M \otimes p_{H}^{L} \otimes\left(p_{B}^{L} \circ \mu_{B} \circ\left(\bar{\Pi}_{B}^{R} \otimes i_{B}^{L}\right)\right)\right) \circ\left(\left(\left(M \otimes c_{B, H}\right) \circ\left(c_{M, B} \otimes H\right)\right.\right.$ $\left.\left.\circ\left(B \otimes\left(\varphi_{M} \circ c_{M, H}\right) \otimes H\right) \circ\left(\rho_{M} \otimes\left(\left(\bar{\Pi}_{H}^{L} \otimes H\right) \circ \delta_{H} \circ i_{H}^{L}\right)\right)\right) \otimes B_{L}\right)$ (by (13)).

Finally,

$\Omega_{M \otimes\left(H_{L} \otimes B_{L}\right)} \circ\left(\varphi_{M} \otimes p_{H}^{L} \otimes p_{B}^{L}\right)$

$=\left(\left(\varepsilon_{B} \circ \mu_{B}\right) \otimes M \otimes p_{H}^{L} \otimes p_{B}^{L}\right) \circ\left(B \otimes c_{M, B} \otimes H \otimes B\right)$

$\circ\left(\left(\rho_{M} \circ \varphi_{M}\right) \otimes\left(c_{H, B} \circ\left(\mu_{H} \otimes B\right)\right) \otimes B\right) \circ\left(H \otimes c_{H, M} \otimes H \otimes\left(\delta_{B} \circ \Pi_{B}^{L}\right)\right) \circ\left(\left(\left(\mu_{H} \otimes H\right)\right.\right.$

$\left.\left.\circ\left(H \otimes c_{H, H}\right) \circ\left(\left(\delta_{H} \circ \eta_{H}\right) \otimes H\right)\right) \otimes M \otimes H \otimes B\right)$ (by the naturality of $c$, (5) and the condition of left

$H$-module for $M)$

$=\left(\left(\varepsilon_{B} \circ \mu_{B}\right) \otimes M \otimes p_{H}^{L} \otimes p_{B}^{L}\right) \circ\left(B \otimes c_{M, B} \otimes H \otimes B\right) \circ\left(\left(\left(B \otimes \varphi_{M}\right) \circ\left(c_{H, B} \otimes M\right)\right.\right.$

$\left.\left.\circ\left(H \otimes \rho_{M}\right)\right) \otimes\left(c_{H, B} \circ\left(\mu_{H} \otimes B\right)\right) \otimes B\right) \circ\left(H \otimes c_{H, M} \otimes H \otimes\left(\delta_{B} \circ \Pi_{B}^{L}\right)\right)$

$\circ\left(\left(\left(H \otimes \Pi_{H}^{L}\right) \circ \delta_{H}\right) \otimes M \otimes H \otimes B\right)$ (by (10) and (44))

$=\left(\varphi_{M} \otimes\left(p_{H}^{L} \circ \mu_{H}\right) \otimes p_{B}^{L}\right) \circ\left(H \otimes c_{H, M} \otimes H \otimes B\right)$

$\circ\left(\left(\left(H \otimes \Pi_{H}^{L}\right) \circ \delta_{H}\right) \otimes\left(\left(\left(\varepsilon_{B} \circ \mu_{B}\right) \otimes M \otimes H \otimes B\right) \circ\left(B \otimes c_{M, B} \otimes H \otimes B\right)\right.\right.$

$\left.\left.\circ\left(B \otimes M \otimes c_{H, B} \otimes B\right) \circ\left(\rho_{M} \otimes H \otimes\left(\delta_{B} \circ \Pi_{B}^{L}\right)\right)\right)\right)$ (by the naturality of $c$ )

$=\left(\varphi_{M} \otimes\left(p_{H}^{L} \circ \mu_{H}\right) \otimes\left(p_{B}^{L} \circ \mu_{B} \circ\left(\bar{\Pi}_{B}^{R} \otimes B\right)\right)\right) \circ\left(\left(\left(H \otimes c_{H, M} \otimes c_{B, H}\right)\right.\right.$

$\circ\left(\left(\left(\left(H \otimes \Pi_{H}^{L}\right) \circ \delta_{H}\right) \otimes\left(c_{B, M} \circ \rho_{M}\right) \otimes H\right) \otimes B\right)$ (by the naturality of $c$ and (13))

and (74) holds. 
Proposition 3. Let $H$ and $B$ be weak Hopf monoids and let $\left(M, \varphi_{M}, \rho_{M}\right)$ be in ${ }_{H}^{B}$ Long. The morphisms

$$
l_{M}:\left(H_{L} \otimes B_{L}\right) \times M \rightarrow M, \quad r_{M}: M \times\left(H_{L} \otimes B_{L}\right) \rightarrow M
$$

defined by

$$
l_{M}=\left(\left(\varepsilon_{B} \circ \mu_{B}\right) \otimes M\right) \circ\left(B \otimes\left(\rho_{M} \circ \varphi_{M}\right)\right) \circ\left(\left(c_{H, B} \circ\left(i_{H}^{L} \otimes i_{B}^{L}\right)\right) \otimes M\right) \circ \circ j_{\left(H_{L} \otimes B_{L}\right) \otimes M}
$$

and

$$
\begin{aligned}
& r_{M}=\left(\left(\varphi_{M} \circ c_{M, H}\right) \otimes\left(\varepsilon_{B} \circ \mu_{B}\right)\right) \circ\left(M \otimes c_{B, H} \otimes B\right) \circ\left(\left(c_{B, M} \circ \rho_{M}\right) \otimes\left(\bar{\Pi}_{H}^{L} \circ i_{H}^{L}\right) \otimes i_{B}^{L}\right) \circ j_{M \otimes\left(H_{L} \otimes B_{L}\right)} \\
& \text { are natural isomorphisms in }{ }_{H}^{B} \text { Long and satisfy the Triangle Axiom. }
\end{aligned}
$$

Proof. First note that it is easy to show that $l_{M}$ and $r_{M}$ are natural morphisms because (66) holds. The morphisms $l_{M}$ is an isomorphism with inverse

$$
l_{M}^{\prime}=q_{M \otimes\left(H_{L} \otimes B_{L}\right)} \circ\left(p_{H}^{L} \otimes p_{B}^{L} \otimes M\right) \circ\left(H \otimes\left(\rho_{M} \circ \varphi_{M}\right)\right) \circ\left(\left(\delta_{H} \circ \eta_{H}\right) \otimes M\right) .
$$

Indeed, on one hand,

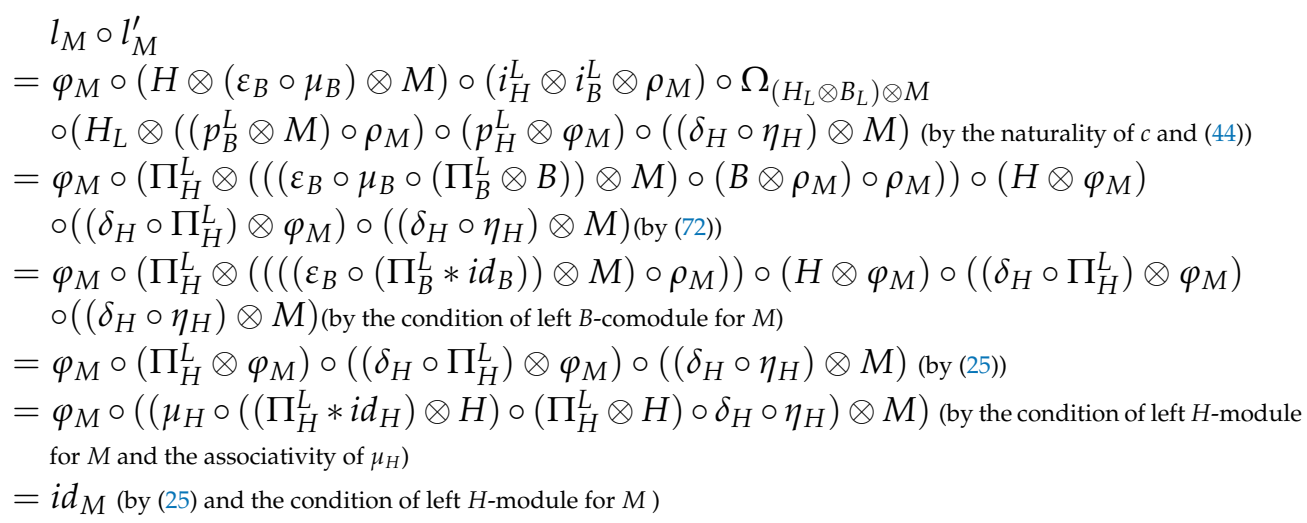
and, on the other hand,

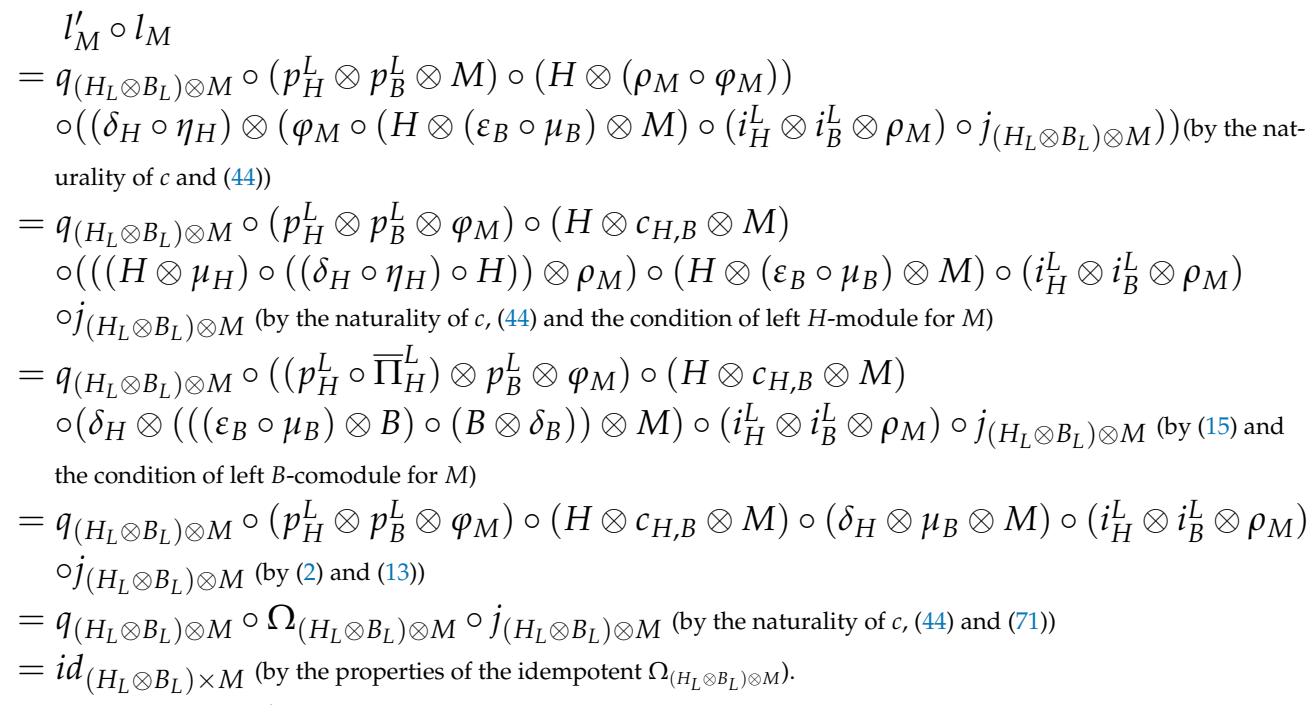

The morphism $l_{M}^{\prime}$ is a morphism of left $H$-modules because

$$
\begin{aligned}
& l_{M}^{\prime} \circ \varphi_{\left(H_{L} \otimes B_{L}\right) \times M} \\
& =q_{\left(H_{L} \otimes B_{L}\right) \otimes M} \circ\left(\left(p_{H}^{L} \circ \mu_{H} \circ\left(H \otimes i_{H}^{L}\right)\right) \otimes B_{L} \otimes \varphi_{M}\right) \circ\left(H \otimes \left(\left(H_{L} \otimes c_{H, B_{L}}\right)\right.\right. \\
& \left.\left.\circ\left(c_{H, H_{L}} \otimes B_{L}\right)\right) \otimes M\right) \circ\left(\delta _ { H } \otimes \left(\left(\Omega_{\left(H_{L} \otimes B_{L}\right) \otimes M} \circ\left(p_{H}^{L} \otimes p_{B}^{L} \otimes \varphi_{M}\right) \circ\left(H \otimes c_{H, B} \otimes M\right)\right.\right.\right. \\
& \left.\left.\left.\circ\left(\left(\delta_{H} \circ \eta_{H}\right) \otimes \rho_{M}\right)\right)\right)\right) \text { (by (44)) } \\
& =q_{\left(H_{L} \otimes B_{L}\right) \otimes M} \circ\left(\left(p_{H}^{L} \circ \mu_{H} \circ\left(H \otimes \Pi_{H}^{L}\right)\right) \otimes p_{B}^{L} \otimes \varphi_{M}\right) \circ\left(H \otimes H \otimes c_{H, B} \otimes \varphi_{M}\right)
\end{aligned}
$$


$\left.\circ\left(H \otimes c_{H, H} \otimes c_{H, B_{L}} \otimes M\right)\right) \circ\left(\delta_{H} \otimes\left(\delta_{H} \circ \eta_{H}\right) \otimes \rho_{M}\right)$ (by (57) and naturality of $\left.c\right)$

$=q_{\left(H_{L} \otimes B_{L}\right) \otimes M} \circ\left(\left(p_{H}^{L} \circ \mu_{H}\right) \otimes p_{B}^{L} \otimes\left(\varphi_{M} \circ\left(\mu_{H} \otimes M\right)\right)\right) \circ\left(H \otimes H \otimes c_{H, B} \otimes H \otimes M\right)$

$\left.\circ\left(H \otimes c_{H, H} \otimes c_{H, B_{L}} \otimes M\right)\right) \circ\left(\delta_{H} \otimes\left(\delta_{H} \circ \eta_{H}\right) \otimes \rho_{M}\right)$ (by (5) and the condition of left $H$-module for $M$ )

$=q_{\left(H_{L} \otimes B_{L}\right) \otimes M} \circ\left(p_{H}^{L} \otimes p_{B}^{L} \otimes \varphi_{M}\right) \circ\left(H \otimes c_{H, B} \otimes M\right)$

$\left.\circ\left(\left(\mu_{H} \otimes \mu_{H}\right) \circ \delta_{H \otimes H} \circ\left(H \otimes \eta_{H}\right)\right) \otimes \rho_{M}\right)$ (by the naturality of $c$ and the associativity of $\mu_{H}$ )

$=q_{\left(H_{L} \otimes B_{L}\right) \otimes M} \circ\left(p_{H}^{L} \otimes p_{B}^{L} \otimes M\right) \circ\left(H \otimes\left(\rho_{M} \circ \varphi_{M}\right)\right) \circ\left(\delta_{H} \otimes M\right)$ (by (a1) of Definition 1, proper(ties of $\eta_{H}$ and (44))

$=q_{\left(H_{L} \otimes B_{L}\right) \otimes M} \circ\left(\left(p_{H}^{L} \circ \bar{\Pi}_{H}^{L}\right) \otimes p_{B}^{L} \otimes M\right) \circ\left(H \otimes\left(\rho_{M} \circ \varphi_{M}\right)\right) \circ\left(\delta_{H} \otimes M\right)$ (by (1))

$=q_{\left(H_{L} \otimes B_{L}\right) \otimes M} \circ\left(p_{H}^{L} \otimes p_{B}^{L} \otimes M\right) \circ\left(H \otimes\left(\rho_{M} \circ \varphi_{M}\right)\right)$

$\circ\left(\left(\left(H \otimes \mu_{H}\right) \circ\left(\left(\delta_{H} \circ \eta_{H}\right) \otimes H\right)\right) \otimes M\right)$ (by (15))

$=l_{M}^{\prime} \circ \varphi_{M}$ (by the condition of left $H$-module for $M$ ).

Therefore, $l_{M}$ is a morphism of left $H$-modules. Moreover, $l_{M}$ is a morphism of left $B$-comodules because,

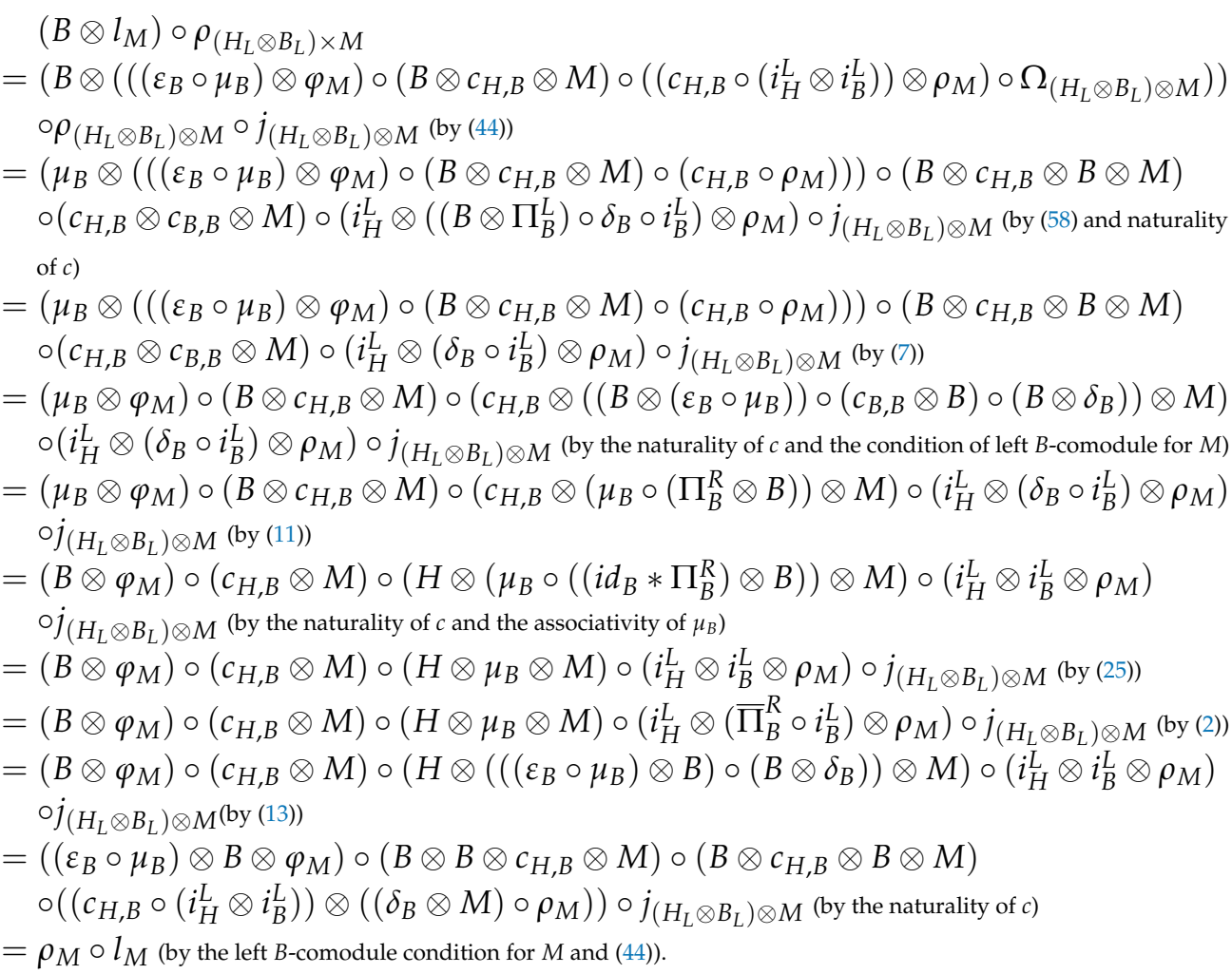

Thus, $l_{M}$ is a morphism in ${ }_{H}^{B}$ Long.

The morphisms $r_{M}$ is an isomorphism with inverse

$r_{M}^{\prime}=q_{\left(H_{L} \otimes B_{L}\right) \otimes M} \circ\left(\varphi_{M} \otimes p_{H}^{L} \otimes p_{B}^{L}\right) \circ\left(H \otimes c_{H, M} \otimes B\right)$

$\circ\left(\left(\delta_{H} \circ \eta_{H}\right) \otimes\left(c_{B, M} \circ\left(\bar{\Pi}_{B}^{R} \otimes M\right) \circ \rho_{M}\right)\right)$.

Indeed: On one hand

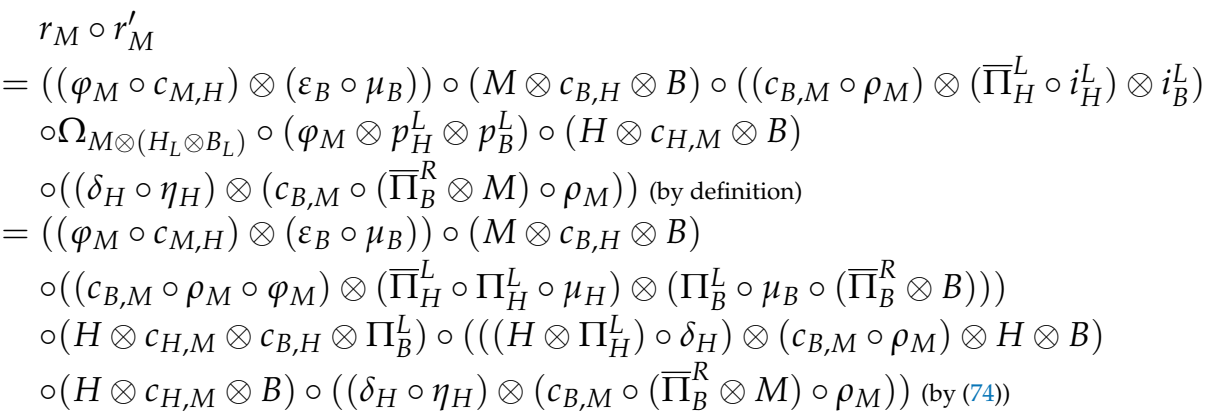




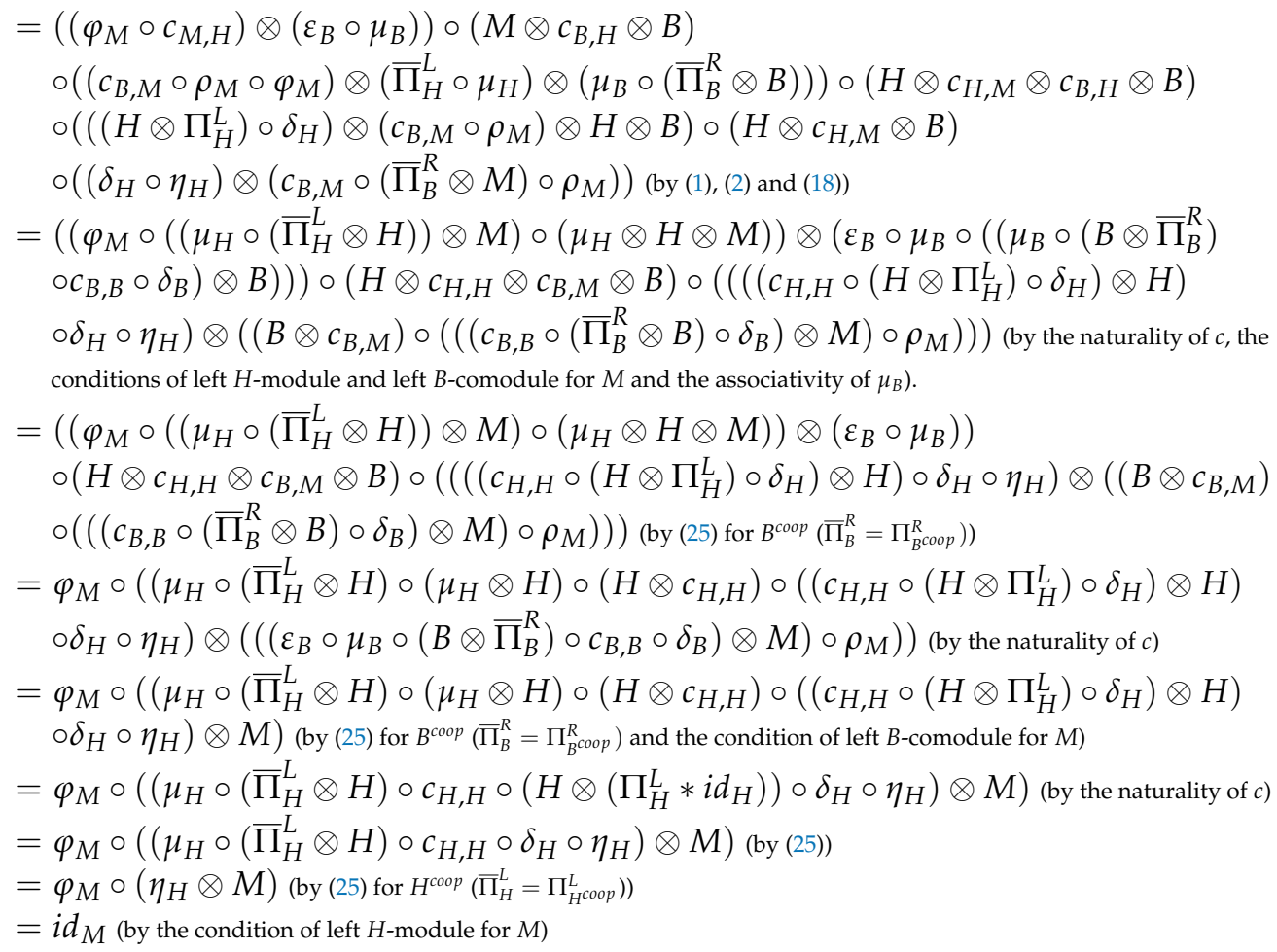

and, on the other hand,

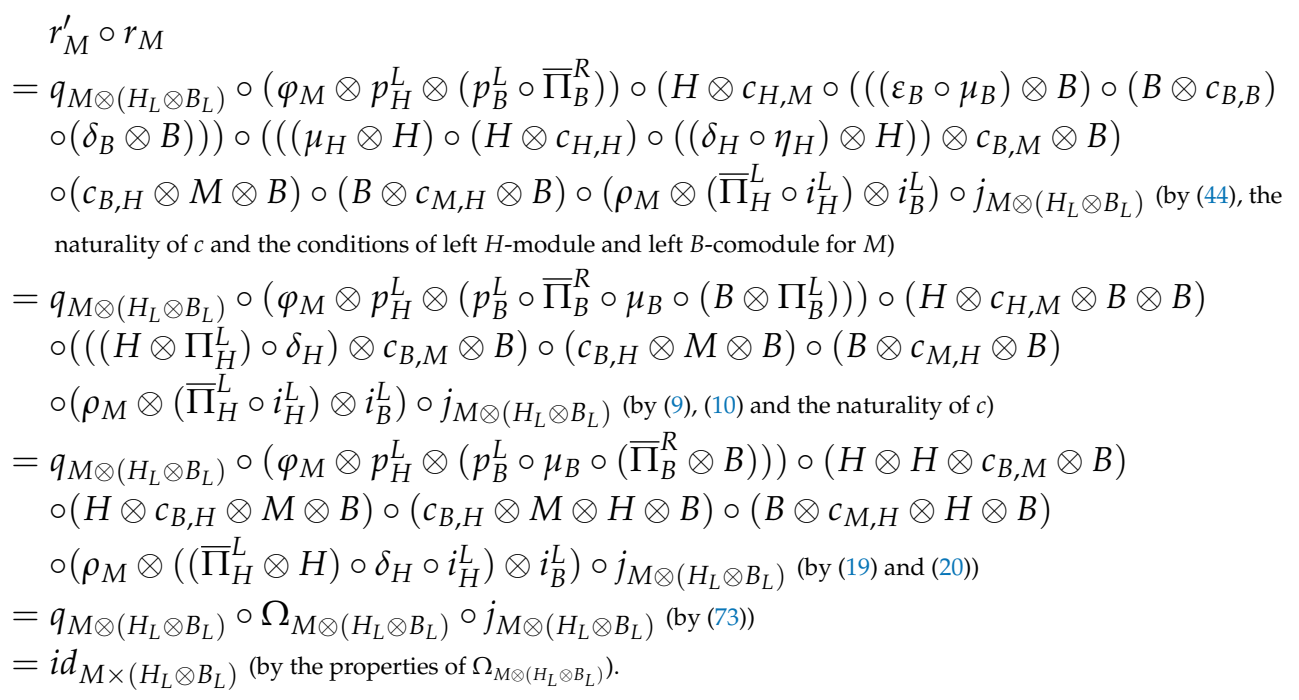

The morphism $r_{M}^{-1}$ is a morphism of left $H$-modules because

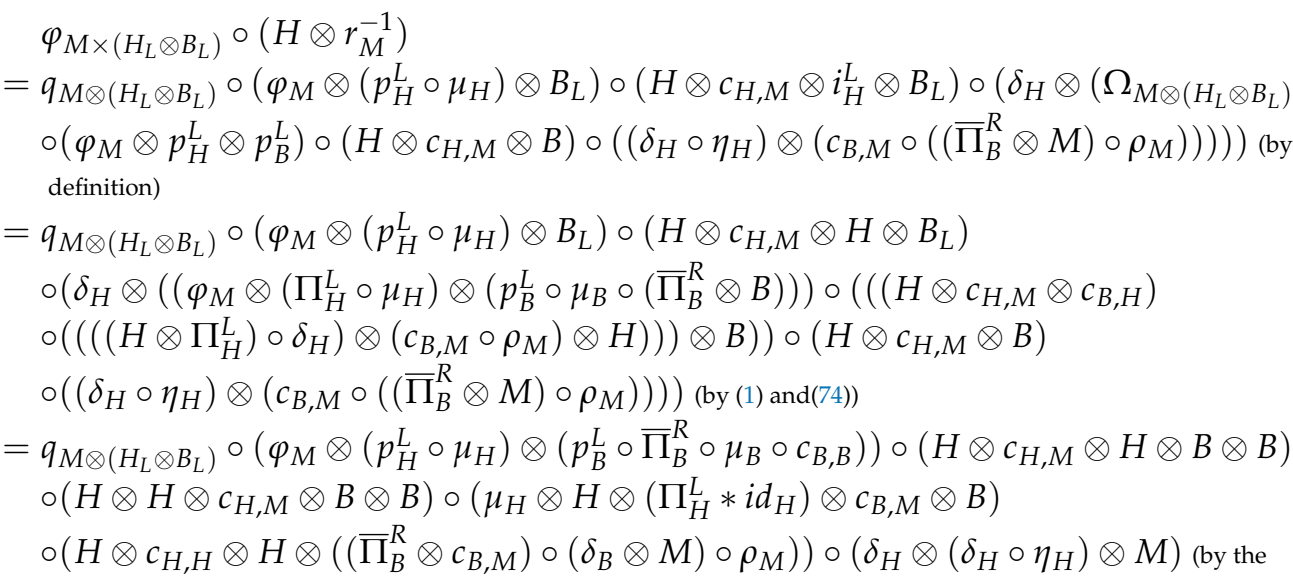




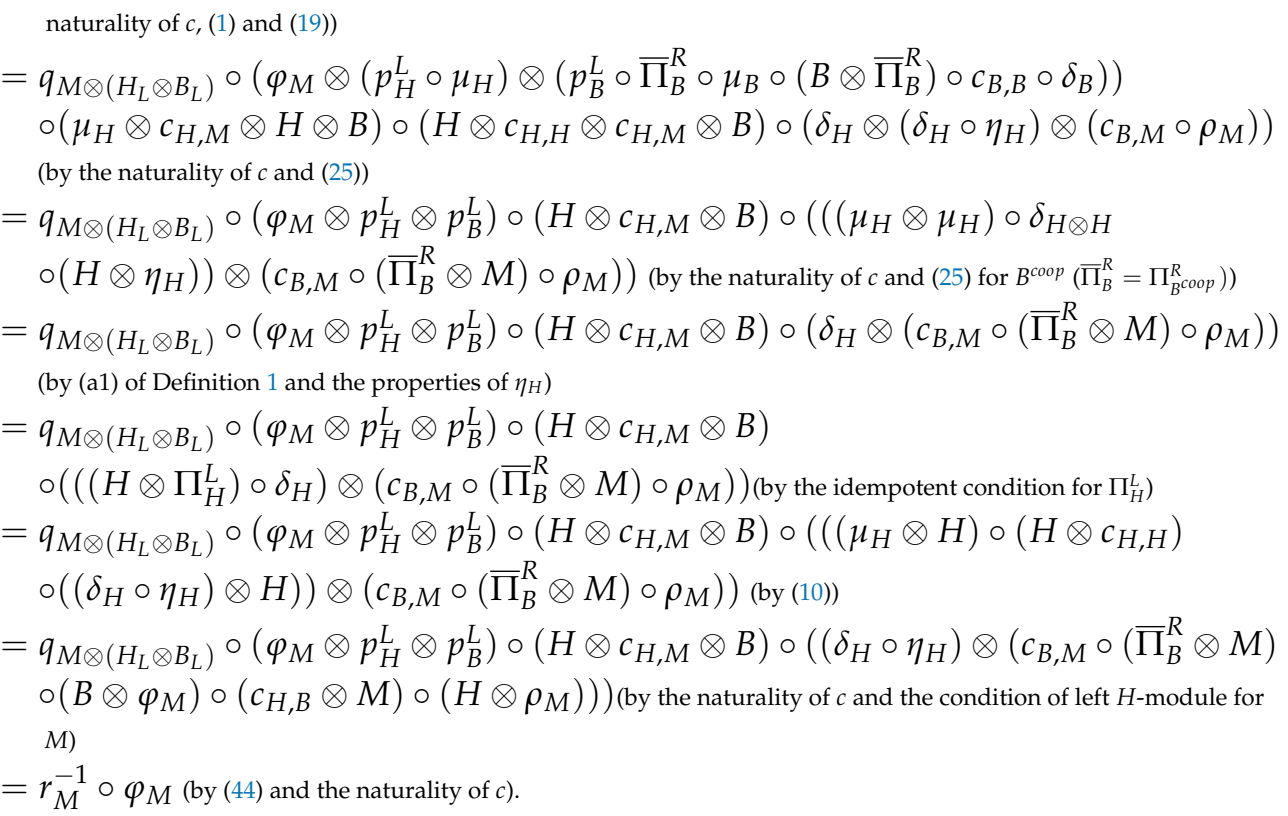

Therefore, $r_{M}$ is a morphism of left $H$-modules. Moreover, $r_{M}$ is a morphism of left $B$-comodules because:

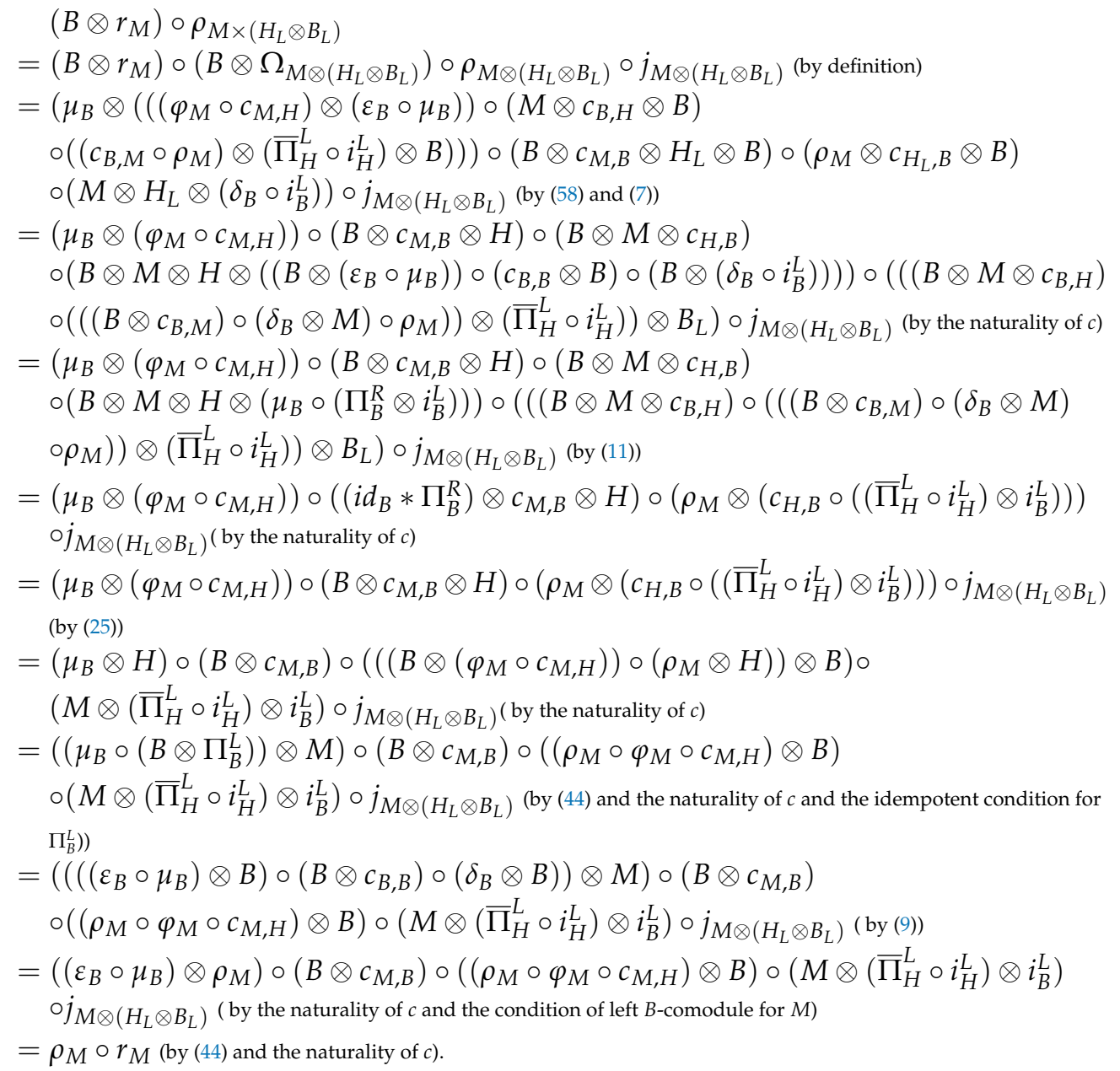

Thus, $r_{M}$ is a morphism in ${ }_{H}^{B}$ Long.

Finally, the Triangle Axiom follows from:

$$
\begin{aligned}
& \left(i d_{M} \times l_{N}\right) \circ a_{M, H_{L} \otimes B_{L}, N} \circ\left(r_{M}^{-1} \times i d_{N}\right) \\
= & q_{M \otimes N} \circ\left(M \otimes l_{N}\right) \circ \Omega_{M \otimes\left(\left(H_{L} \otimes B_{L}\right) \times N\right)} \circ\left(M \otimes q_{\left(H_{L} \otimes B_{L}\right) \otimes N}\right) \circ\left(j_{M \otimes\left(H_{L} \otimes B_{L}\right)} \otimes N\right)
\end{aligned}
$$




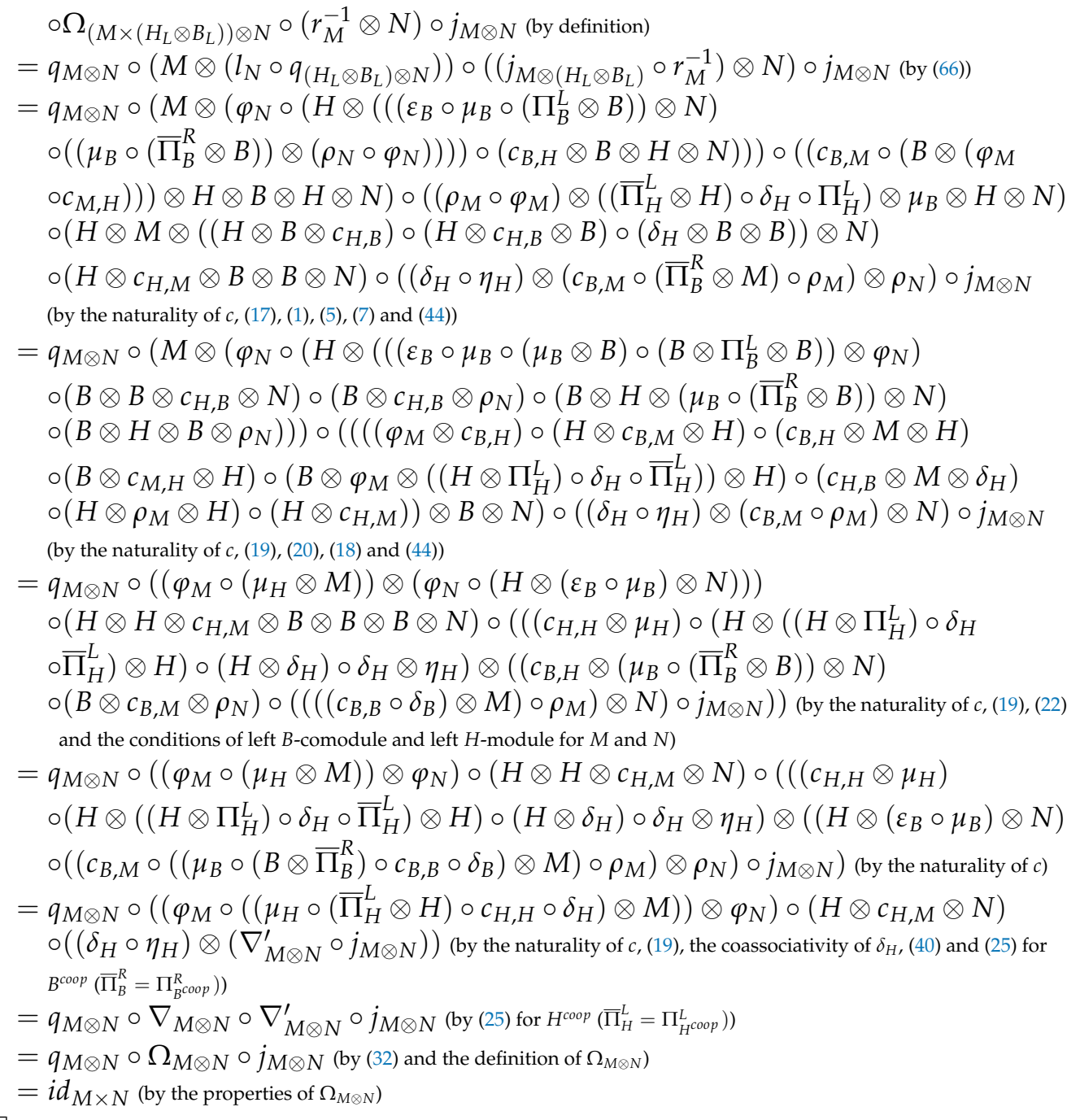

Theorem 1. Let $H$ and $B$ be weak Hopf monoids. The category ${ }_{H}^{B}$ Long is monoidal.

Proof. The proof is a direct consequence of Propositions 2 and 3.

\section{Quasitriangular Weak Hopf Monoids and Long Dimodules}

In the first part of this section, we give a summary about quasitriangular and coquasitriangular weak Hopf monoids in a monoidal setting. The complete details for the quasitriangular context can be found in [21]. By reversing arrows, it is easy to get the corresponding results for coquasitriangular Hopf monoids.

Let $H$ be a weak Hopf monoid in C. By [21] [Lemma 3.1] we have that the morphisms

$$
\begin{gathered}
\Omega_{H}^{1}=\mu_{H \otimes H} \circ\left(\left(c_{H, H} \circ \delta_{H} \circ \eta_{H}\right) \otimes H \otimes H\right): H \otimes H \rightarrow H \otimes H, \\
\Omega_{H}^{2}=\mu_{H \otimes H} \circ\left(H \otimes H \otimes\left(\delta_{H} \circ \eta_{H}\right)\right): H \otimes H \rightarrow H \otimes H, \\
\Omega_{H}^{3}=\mu_{H \otimes H} \circ\left(H \otimes H \otimes\left(c_{H, H} \circ \delta_{H} \circ \eta_{H}\right)\right): H \otimes H \rightarrow H \otimes H, \\
\Omega_{H}^{4}=\mu_{H \otimes H} \circ\left(\left(\delta_{H} \circ \eta_{H}\right) \otimes H \otimes H\right): H \otimes H \rightarrow H \otimes H,
\end{gathered}
$$

are idempotent and $\Omega_{H}^{1} \circ \Omega_{H}^{2}=\Omega_{H}^{2} \circ \Omega_{H}^{1}, \Omega_{H}^{3} \circ \Omega_{H}^{4}=\Omega_{H}^{4} \circ \Omega_{H}^{3}$. Also, by ([21], Remark 3.2), we have that the following identities

$$
\Omega_{H}^{1}=\left(H \otimes \mu_{H}\right) \circ\left(\left(c_{H, H} \circ\left(\bar{\Pi}_{H}^{L} \otimes H\right) \circ \delta_{H}\right) \otimes H\right)
$$




$$
\begin{gathered}
=c_{H, H} \circ\left(H \otimes\left(\mu_{H} \circ\left(\Pi_{H}^{L} \otimes H\right)\right)\right) \circ\left(\delta_{H} \otimes H\right) \circ c_{H, H}, \\
\Omega_{H}^{2}=\left(\mu_{H} \otimes H\right) \circ\left(H \otimes\left(\left(\Pi_{H}^{R} \otimes H\right) \circ \delta_{H}\right)\right. \\
=\left(H \otimes\left(\mu_{H} \circ c_{H, H} \circ\left(\bar{\Pi}_{H}^{R} \otimes H\right)\right)\right) \circ\left(\delta_{H} \otimes H\right), \\
\Omega_{H}^{3}=\left(\mu_{H} \otimes H\right) \circ\left(H \otimes\left(c_{H, H} \circ\left(H \otimes \bar{\Pi}_{H}^{R}\right) \circ \delta_{H}\right)\right) \\
=c_{H, H} \circ\left(\left(\mu_{H} \circ\left(H \otimes \Pi_{H}^{R}\right)\right) \otimes H\right) \circ\left(H \otimes \delta_{H}\right) \circ c_{H, H}, \\
\Omega_{H}^{4}=\left(H \otimes\left(\mu_{H} \circ\left(\Pi_{H}^{L} \otimes H\right)\right) \circ\left(\delta_{H} \otimes H\right)\right. \\
=\left(\left(\mu_{H} \circ c_{H, H} \circ\left(H \otimes \bar{\Pi}_{H}^{L}\right)\right) \otimes H\right) \circ\left(H \otimes \delta_{H}\right)
\end{gathered}
$$

hold. Moreover, if we define $\Omega_{H}$ and $\Omega_{H}^{\prime}$ by

$$
\Omega_{H}=\Omega_{H}^{2} \circ \Omega_{H}^{1}, \quad \Omega_{H}^{\prime}=\Omega_{H}^{4} \circ \Omega_{H}^{3} .
$$

we have that $\Omega_{H}$ and $\Omega_{H}^{\prime}$ are idempotent morphisms and, if $\alpha: A \rightarrow H \otimes H$ is a morphism in $\mathrm{C}$,

$$
\Omega_{H} \circ \alpha=\alpha \Leftrightarrow \Omega_{H}^{1} \circ \alpha=\alpha \text { and } \Omega_{H}^{2} \circ \alpha=\alpha,
$$

and

$$
\Omega_{H}^{\prime} \circ \alpha=\alpha \Leftrightarrow \Omega_{H}^{3} \circ \alpha=\alpha \text { and } \Omega_{H}^{4} \circ \alpha=\alpha .
$$

Similarly, the morphisms

$$
\begin{gathered}
\Gamma_{H}^{1}=\left(\left(\varepsilon_{H} \circ \mu_{H} \circ c_{H, H}\right) \otimes H \otimes H\right) \circ \delta_{H \otimes H}: H \otimes H \rightarrow H \otimes H, \\
\Gamma_{H}^{2}=\left(H \otimes H \otimes\left(\varepsilon_{H} \circ \mu_{H}\right)\right) \circ \delta_{H \otimes H}: H \otimes H \rightarrow H \otimes H, \\
\Gamma_{H}^{3}=\left(H \otimes H \otimes\left(\varepsilon_{H} \circ \mu_{H} \circ c_{H, H}\right)\right) \circ \delta_{H \otimes H}: H \otimes H \rightarrow H \otimes H, \\
\Gamma_{H}^{4}=\left(\left(\varepsilon_{H} \circ \mu_{H}\right) \otimes H \otimes H\right) \circ \delta_{H \otimes H}: H \otimes H \rightarrow H \otimes H,
\end{gathered}
$$

are idempotent and $\Gamma_{H}^{1} \circ \Gamma_{H}^{2}=\Gamma_{H}^{2} \circ \Gamma_{H}^{1}, \Gamma_{H}^{3} \circ \Gamma_{H}^{4}=\Gamma_{H}^{4} \circ \Gamma_{H}^{3}$. Also, the following identities hold:

$$
\begin{gathered}
\Gamma_{H}^{1}=\left(\left(\mu_{H} \circ\left(\bar{\Pi}_{H}^{R} \otimes H\right) \circ c_{H, H}\right) \otimes H\right) \circ\left(H \otimes \delta_{H}\right) \\
=c_{H, H} \circ\left(\left(\mu_{H} \circ\left(H \otimes \Pi_{H}^{L}\right)\right) \otimes H\right) \circ\left(H \otimes \delta_{H}\right) \circ c_{H, H}, \\
\Gamma_{H}^{2}=\left(H \otimes\left(\mu_{H} \circ\left(\Pi_{H}^{R} \otimes H\right)\right)\right) \circ\left(\delta_{H} \otimes H\right) \\
=\left(\mu_{H} \otimes H\right) \circ\left(H \otimes\left(\left(\bar{\Pi}_{H}^{L} \otimes H\right) \circ c_{H, H} \circ \delta_{H}\right)\right), \\
\Gamma_{H}^{3}=\left(H \otimes\left(\mu_{H} \circ\left(H \otimes \bar{\Pi}_{H}^{L}\right) \circ c_{H, H}\right)\right) \circ\left(\delta_{H} \otimes H\right) \\
=c_{H, H} \circ\left(H \otimes\left(\mu_{H} \circ\left(\Pi_{H}^{R} \otimes H\right)\right)\right) \circ\left(\delta_{H} \otimes H\right) \circ c_{H, H}, \\
\Gamma_{H}^{4}=\left(\mu_{H} \otimes H\right) \circ\left(H \otimes\left(\left(\Pi_{H}^{L} \otimes H\right) \circ \delta_{H}\right)\right) \\
=\left(H \otimes \mu_{H}\right) \circ\left(\left(\left(H \otimes \bar{\Pi}_{H}^{R}\right) \circ c_{H, H} \circ \delta_{H}\right) \otimes H\right) .
\end{gathered}
$$

Moreover, if we define $\Gamma_{H}$ and $\Gamma_{H}^{\prime}$ by

$$
\Gamma_{H}=\Gamma_{H}^{2} \circ \Gamma_{H}^{1}, \quad \Gamma_{H}^{\prime}=\Gamma_{H}^{4} \circ \Gamma_{H}^{3},
$$

we have that $\Gamma_{H}$ and $\Gamma_{H}^{\prime}$ are idempotent morphisms and, if $\beta: H \otimes H \rightarrow A$ is a morphism in $\mathrm{C}$,

$$
\beta \circ \Gamma_{H}=\beta \Leftrightarrow \beta \circ \Gamma_{H}^{1}=\beta \text { and } \beta \circ \Gamma_{H}^{2}=\beta
$$

and

$$
\beta \circ \Gamma_{H}^{\prime}=\beta \Leftrightarrow \beta \circ \Gamma_{H}^{3}=\beta \text { and } \beta \circ \Gamma_{H}^{4}=\beta
$$


hold.

The following definition is the categorical monoidal version of the definition of quasitriangular weak Hopf monoid introduced by Nikshych, Turaev and Vainerman in [22].

Definition 5. Let $H$ be a weak Hopf monoid. Let $\Omega_{H}$ and $\Omega_{H}^{\prime}$ be the idempotent morphisms defined in (83). We will say that $H$ is a quasitriangular weak Hopf algebra if there exists a morphism $\sigma: K \rightarrow H \otimes H$ in $C$ satisfying the following conditions:

(d1) $\Omega_{H} \circ \sigma=\sigma$.

(d2) $\left(\delta_{H} \otimes H\right) \circ \sigma=\left(H \otimes \mu_{H}\right) \circ\left(H \otimes c_{H, H} \otimes H\right) \circ(\sigma \otimes \sigma)$.

(d3) $\left(H \otimes \delta_{H}\right) \circ \sigma=\left(\mu_{H} \otimes c_{H, H}\right) \circ\left(H \otimes c_{H, H} \otimes H\right) \circ(\sigma \otimes \sigma)$.

(d4) $\mu_{H \otimes H} \circ\left(\sigma \otimes \delta_{H}\right)=\mu_{H \otimes H} \circ\left(\left(c_{H, H} \circ \delta_{H}\right) \otimes \sigma\right)$.

(d5) There exists a morphism $\bar{\sigma}: K \rightarrow H \otimes H$ such that:

(d5.1) $\Omega_{H}^{\prime} \circ \bar{\sigma}=\bar{\sigma}$.

(d5.2) $\sigma * \bar{\sigma}=c_{H, H} \circ \delta_{H} \circ \eta_{H}$

(d5.3) $\bar{\sigma} * \sigma=\delta_{H} \circ \eta_{H}$.

We will say that a quasitriangular weak Hopf monoid $H$ is triangular if moreover $\bar{\sigma}=$ $c_{H, H} \circ \sigma$.

For any quasitriangular weak Hopf monoid the morphism $\bar{\sigma}$ is unique and by [21] [Lemma 3.5] the equalities

$$
\begin{gathered}
\sigma * \bar{\sigma} * \sigma=\sigma, \bar{\sigma} * \sigma * \bar{\sigma}=\bar{\sigma}, \\
\left(\varepsilon_{H} \otimes H\right) \circ \sigma=\left(H \otimes \varepsilon_{H}\right) \circ \sigma=\eta_{H}, \\
\mu_{H} \circ c_{H, H} \circ\left(H \otimes \Pi_{H}^{L}\right) \circ \sigma=\eta_{H}=\mu_{H} \circ\left(\bar{\Pi}_{H}^{L} \otimes H\right) \circ \sigma, \\
\mu_{H} \circ\left(H \otimes \Pi_{H}^{R}\right) \circ \sigma=\eta_{H}=\mu_{H} \circ c_{H, H} \circ\left(\bar{\Pi}_{H}^{R} \otimes H\right) \circ \sigma, \\
\mu_{H} \circ\left(H \otimes \bar{\Pi}_{H}^{R}\right) \circ \bar{\sigma}=\eta_{H}=\mu_{H} \circ c_{H, H} \circ\left(\Pi_{H}^{R} \otimes H\right) \circ \bar{\sigma}, \\
\mu_{H} \circ\left(\Pi_{H}^{L} \otimes H\right) \circ \bar{\sigma}=\eta_{H}=\mu_{H} \circ c_{H, H} \circ\left(H \otimes \bar{\Pi}_{H}^{L}\right) \circ \bar{\sigma},
\end{gathered}
$$

hold.

Lemma 7. Let $H$ be a quasitriangular weak Hopf monoid in C. Then,

$$
\begin{gathered}
\left(\Pi_{H}^{R} \otimes H\right) \circ \sigma=\left(H \otimes \bar{\Pi}_{H}^{R}\right) \circ \sigma=\left(H \otimes \Pi_{H}^{L}\right) \circ \bar{\sigma}=\left(\bar{\Pi}_{H}^{L} \otimes H\right) \circ \bar{\sigma}=\delta_{H} \circ \eta_{H}, \\
\left(\Pi_{H}^{L} \otimes H\right) \circ \sigma=\left(H \otimes \bar{\Pi}_{H}^{L}\right) \circ \sigma=\left(H \otimes \Pi_{H}^{R}\right) \circ \bar{\sigma}=\left(\bar{\Pi}_{H}^{R} \otimes H\right) \circ \bar{\sigma}=c_{H, H} \circ \delta_{H} \circ \eta_{H} .
\end{gathered}
$$

Proof. We will prove (103). The proof for (104) is similar and we lend the details to the reader. First note that the identities

$$
\begin{aligned}
& \left(\Pi_{H}^{R} \otimes H\right) \circ \mu_{H \otimes H} \circ\left(\sigma \otimes \delta_{H}\right) \\
= & \left(\left(\Pi_{H}^{R} \circ \mu_{H} \circ\left(\Pi_{H}^{R} \otimes H\right)\right) \otimes \mu_{H}\right) \circ\left(H \otimes c_{H, H} \otimes H\right) \circ\left(\sigma \otimes \delta_{H}\right) \text { (by (5)) } \\
= & \left(\left(\left(\Pi_{H}^{R} \otimes\left(\varepsilon_{H} \circ \mu_{H}\right)\right) \circ\left(c_{H, H} \otimes H\right) \circ\left(H \otimes \delta_{H}\right)\right) \otimes \mu_{H}\right) \circ\left(H \otimes c_{H, H} \otimes H\right) \circ\left(\sigma \otimes \delta_{H}\right) \\
& (\text { by }(11)) \\
= & \left(\Pi_{H}^{R} \otimes\left(\left(\left(\varepsilon_{H} \circ \mu_{H}\right) \otimes\left(\mu_{H} \circ c_{H, H}\right)\right) \circ\left(H \otimes \delta_{H} \otimes H\right) \circ\left(H \otimes c_{H, H}\right) \circ(\sigma \otimes H)\right)\right) \circ \delta_{H} \\
& \text { (by the naturality of c and the coassociativity of } \left.\delta_{H}\right) \\
= & \left.\left.\left(\Pi_{H}^{R} \otimes\left(\mu_{H} \circ c_{H, H} \circ\left(\left(\mu_{H} \circ\left(\bar{\Pi}_{H}^{R} \otimes H\right)\right) \otimes H\right)\right)\right) \circ\left(H \otimes c_{H, H}\right) \circ(\sigma \otimes H)\right)\right) \circ \delta_{H} \text { (by (13)) } \\
= & \left(\Pi_{H}^{R} \otimes\left(\mu_{H} \circ\left(\left(\mu_{H} \circ c_{H, H} \circ\left(\Pi_{H}^{R} \otimes H\right) \circ \sigma\right) \circ H\right)\right)\right) \circ \delta_{H} \text { (by the naturality of c and the associativity } \\
& \text { of } \left.\mu_{H}\right) \\
= & \left.\left(\Pi_{H}^{R} \otimes H\right) \circ \delta_{H} \text { (by (100) and the properties of } \eta_{H}\right),
\end{aligned}
$$


hold. Then, we obtain the identity

$$
\left(\Pi_{H}^{R} \otimes H\right) \circ \mu_{H \otimes H} \circ\left(\sigma \otimes \delta_{H}\right)=\left(\Pi_{H}^{R} \otimes H\right) \circ \delta_{H}
$$

and, as a consequence,

$$
\left(\Pi_{H}^{R} \otimes H\right) \circ \sigma \stackrel{(84)}{=}\left(\Pi_{H}^{R} \otimes H\right) \circ \Omega_{H}^{2} \circ \sigma \stackrel{(105)}{=}\left(\Pi_{H}^{R} \otimes H\right) \circ \delta_{H} \circ \eta_{H} \stackrel{(17)}{=} \delta_{H} \circ \eta_{H} .
$$

Also,

$$
\begin{aligned}
& \left(H \otimes \Pi_{H}^{R}\right) \circ \mu_{H \otimes H} \circ\left(\sigma \otimes \delta_{H}\right) \\
= & \left(\mu_{H} \otimes\left(\Pi_{H}^{R} \circ \mu_{H} \circ\left(\bar{\Pi}_{H}^{R} \otimes H\right)\right)\right) \circ\left(H \otimes c_{H, H} \otimes H\right) \circ\left(\sigma \otimes \delta_{H}\right) \text { (by (1) and (6)) } \\
= & \left(\mu_{H} \otimes\left(\left(\left(\varepsilon_{H} \circ \mu_{H}\right) \otimes \Pi_{H}^{R}\right) \circ \delta_{H}\right)\right) \circ\left(H \otimes c_{H, H} \otimes H\right) \circ\left(\sigma \otimes \delta_{H}\right) \text { (by (13)) } \\
= & \left(\mu_{H} \otimes \Pi_{H}^{R}\right) \circ\left(H \otimes\left(\left(H \otimes\left(\varepsilon_{H} \circ \mu_{H}\right)\right) \circ\left(c_{H, H} \otimes H\right) \circ\left(H \otimes \delta_{H}\right)\right) \otimes H\right) \circ\left(\sigma \otimes \delta_{H}\right) \\
& \text { (by the coassociativity of } \left.\delta_{H}\right) \\
= & \left.\left(\mu_{H} \otimes \Pi_{H}^{R}\right) \circ\left(\left(\mu_{H} \circ\left(H \otimes \Pi_{H}^{R}\right) \circ \sigma\right) \otimes \delta_{H}\right) \text { (by (11) and the associativity of } \mu_{H}\right) \\
= & \left.\left(H \otimes \Pi_{H}^{R}\right) \circ \delta_{H} \text { (by (100) and the properties of } \eta_{H}\right),
\end{aligned}
$$

hold. Then, we obtain the identity

$$
\left(H \otimes \Pi_{H}^{R}\right) \circ \mu_{H \otimes H} \circ\left(\sigma \otimes \delta_{H}\right)=\left(H \otimes \Pi_{H}^{R}\right) \circ \delta_{H}
$$

and, as a consequence,

$\left(H \otimes \bar{\Pi}_{H}^{R}\right) \circ \sigma \stackrel{(2)}{=}\left(H \otimes \bar{\Pi}_{H}^{R}\right) \circ\left(H \otimes \Pi_{H}^{R}\right) \circ \sigma \stackrel{(84)}{=}\left(H \otimes \bar{\Pi}_{H}^{R}\right) \circ\left(H \otimes \Pi_{H}^{R}\right) \circ \Omega_{H}^{2} \circ \sigma \stackrel{(106)}{=}\left(H \otimes \bar{\Pi}_{H}^{R}\right) \circ\left(H \otimes \Pi_{H}^{R}\right) \circ \delta_{H} \circ \eta_{H}$

$$
\stackrel{(2)}{=}\left(H \otimes \bar{\Pi}_{H}^{R}\right) \circ \delta_{H} \circ \eta_{H} \stackrel{(17)}{=} \delta_{H} \circ \eta_{H} .
$$

On the other hand,

$$
\begin{aligned}
& \left(\Pi_{H}^{L} \otimes H\right) \circ \mu_{H \otimes H} \circ\left(\delta_{H} \otimes \bar{\sigma}\right) \\
= & \left(\left(\Pi_{H}^{L} \circ \mu_{H} \circ\left(H \otimes \bar{\Pi}_{H}^{L}\right)\right) \otimes \mu_{H}\right) \circ\left(H \otimes c_{H, H} \otimes H\right) \circ\left(\delta_{H} \otimes \bar{\sigma}\right) \text { (by (1) and (6)) } \\
= & \left.\left(\left(\left(\Pi_{H}^{L} \otimes\left(\varepsilon_{H} \circ \mu_{H}\right)\right) \circ\left(\delta_{H} \otimes H\right)\right)\right) \otimes \mu_{H}\right) \circ\left(H \otimes c_{H, H} \otimes H\right) \circ\left(\delta_{H} \otimes \bar{\sigma}\right) \text { (by (14)) } \\
= & \left(\Pi_{H}^{L} \otimes\left(\left(\left(\varepsilon_{H} \circ \mu_{H}\right) \otimes \mu_{H}\right) \circ\left(H \otimes c_{H, H} \otimes H\right) \circ\left(\delta_{H} \otimes \bar{\sigma}\right)\right) \circ \delta_{H} \text { (by the coassociativity of } \delta_{H}\right) \\
= & \left(\Pi_{H}^{L} \otimes\left(\mu_{H} \circ\left(H \otimes\left(\mu_{H} \circ\left(\Pi_{H}^{L} \otimes H\right) \circ \bar{\sigma}\right)\right)\right) \circ \delta_{H}\left(\text { by (9) and the associativity of } \mu_{H}\right)\right. \\
= & \left.\left(\Pi_{H}^{L} \otimes H\right) \circ \delta_{H} \text { (by (102) and the properties of } \eta_{H}\right),
\end{aligned}
$$

hold. Then, we obtain the identity

$$
\left(\Pi_{H}^{L} \otimes H\right) \circ \mu_{H \otimes H} \circ\left(\delta_{H} \otimes \bar{\sigma}\right)=\left(\Pi_{H}^{L} \otimes H\right) \circ \delta_{H}
$$

and, consequently,

$\left.\left(\bar{\Pi}_{H}^{L}\right) \otimes H\right) \circ \bar{\sigma} \stackrel{(2)}{=}\left(\bar{\Pi}_{H}^{L} \otimes H\right) \circ\left(\Pi_{H}^{L} \otimes H\right) \circ \bar{\sigma} \stackrel{(85)}{=}\left(\bar{\Pi}_{H}^{L} \otimes H\right) \circ\left(\Pi_{H}^{L} \otimes H\right) \circ \Omega_{H}^{4} \circ \bar{\sigma} \stackrel{(107)}{=}\left(\bar{\Pi}_{H}^{L} \otimes H\right) \circ\left(\Pi_{H}^{L} \otimes H\right) \circ \delta_{H} \circ \eta_{H}$

$$
\stackrel{(2)}{=}\left(\bar{\Pi}_{H}^{L} \otimes H\right) \circ \delta_{H} \circ \eta_{H} \stackrel{(17)}{=} \delta_{H} \circ \eta_{H} .
$$

Finally,

$\left(H \otimes \Pi_{H}^{L}\right) \circ \mu_{H \otimes H} \circ\left(\delta_{H} \otimes \bar{\sigma}\right)$

$=\left(\mu_{H} \otimes\left(\Pi_{H}^{L} \circ \mu_{H} \circ\left(H \otimes \Pi_{H}^{L}\right)\right)\right) \circ\left(H \otimes c_{H, H} \otimes H\right) \circ\left(\delta_{H} \otimes \bar{\sigma}\right)$ (by (5))

$=\left(\mu_{H} \otimes\left(\left(\left(\varepsilon_{H} \circ \mu_{H}\right) \otimes \Pi_{H}^{L}\right) \circ\left(H \otimes c_{H, H}\right) \circ\left(\delta_{H} \otimes H\right)\right)\right) \circ\left(H \otimes c_{H, H} \otimes H\right) \circ\left(\delta_{H} \otimes \bar{\sigma}\right)$ (by (9))

$=\left(\left(\left(\left(\mu_{H} \circ c_{H, H}\right) \otimes\left(\varepsilon_{H} \circ \mu_{H}\right)\right) \circ\left(H \otimes \delta_{H} \otimes H\right) \circ\left(c_{H, H} \otimes H\right) \circ(H \otimes \bar{\sigma})\right) \otimes \Pi_{H}^{L}\right) \circ \delta_{H}$ (by the naturality of $c$ and the coassociativity of $\delta_{H}$ )

$=\left(\left(\mu_{H} \circ c_{H, H} \circ\left(H \otimes \mu_{H}\right) \circ\left(c_{H, H} \otimes \bar{\Pi}_{H}^{L}\right) \circ(H \otimes \bar{\sigma})\right) \otimes \Pi_{H}^{L}\right) \circ \delta_{H}($ by (14))

$=\left(\left(\mu_{H} \circ\left(H \otimes\left(\mu_{H} \circ c_{H, H} \circ\left(H \otimes \bar{\Pi}_{H}^{L}\right) \circ \bar{\sigma}\right)\right)\right) \otimes \Pi_{H}^{L}\right) \circ \delta_{H}$ (by the naturality of $c$ and the associativity of $\left.\mu_{H}\right)$ 


$$
\left.=\left(H \otimes \Pi_{H}^{L}\right) \circ \delta_{H} \text { (by (102) and the properties of } \eta_{H}\right),
$$

hold. Then, we obtain the identity

$$
\left(H \otimes \Pi_{H}^{L}\right) \circ \mu_{H \otimes H} \circ\left(\delta_{H} \otimes \bar{\sigma}\right)=\left(H \otimes \Pi_{H}^{L}\right) \circ \delta_{H}
$$

and, consequently,

$$
\left(H \otimes \Pi_{H}^{L}\right) \circ \bar{\sigma} \stackrel{(85)}{=}\left(H \otimes \Pi_{H}^{L}\right) \circ \Omega_{H}^{4} \circ \bar{\sigma} \stackrel{(108)}{=}\left(H \otimes \Pi_{H}^{L}\right) \circ \delta_{H} \circ \eta_{H} \stackrel{(17)}{=} \delta_{H} \circ \eta_{H}
$$

By reversing arrows in Definition 5 we get the definition of coquasitriangular weak Hopf monoid.

Definition 6. Let B be a weak Hopf monoid. Let $\Gamma_{B}$ and $\Gamma_{B}^{\prime}$ be the idempotent morphisms defined in (94). We will say that $B$ is a coquasitriangular weak Hopf algebra if there exists a morphism $\omega: B \otimes B \rightarrow K$ in $C$ satisfying the following conditions:

(e1) $\omega \circ \Gamma_{B}=\omega$.

(e2) $\omega \circ\left(\mu_{B} \otimes B\right)=(\omega \otimes \omega) \circ\left(B \otimes c_{B, B} \otimes B\right) \circ\left(B \otimes B \otimes \delta_{B}\right)$.

(e3) $\omega \circ\left(B \otimes \mu_{B}\right)=(\omega \otimes \omega) \circ\left(B \otimes c_{B, B} \otimes B\right) \circ\left(\delta_{B} \otimes c_{B, B}\right)$.

(e4) $\left(\omega \otimes \mu_{B}\right) \circ \delta_{B \otimes B}=\left(\left(\mu_{B} \circ c_{B, B}\right) \otimes \omega\right) \circ \delta_{B \otimes B}$.

(e5) There exists a morphism $\bar{\omega}: B \otimes B \rightarrow$ such that:

(e5.1) $\bar{\omega} \circ \Gamma_{B}^{\prime}=\bar{\omega}$.

(e5.2) $\omega * \bar{\omega}=\varepsilon_{B} \circ \mu_{B} \circ c_{B, B}$.

(e5.3) $\bar{\omega} * \omega=\varepsilon_{B} \circ \mu_{B}$.

As a consequence of this definition, we obtain that $\bar{\omega}$ is unique and the equalities

$$
\omega * \bar{\omega} * \omega=\omega, \quad \bar{\omega} * \omega * \bar{\omega}=\bar{\omega},
$$

hold.

We will say that a coquasitriangular weak Hopf monoid $B$ is cotriangular if moreover $\bar{\omega}=$ $\omega \circ c_{B, B}$.

Lemma 8. For any coquasitriangular weak Hopf monoid B, the following equalities

$$
\begin{gathered}
\omega \circ\left(\eta_{B} \otimes B\right)=\omega \circ\left(B \otimes \eta_{B}\right)=\varepsilon_{B}, \\
\omega \circ\left(B \otimes \Pi_{B}^{L}\right) \circ c_{B, B} \circ \delta_{B}=\varepsilon_{B}=\omega \circ\left(\bar{\Pi}_{B}^{R} \otimes B\right) \circ \delta_{B}, \\
\omega \circ\left(B \otimes \Pi_{B}^{R}\right) \circ \delta_{B}=\varepsilon_{B}=\omega \circ\left(\bar{\Pi}_{B}^{L} \otimes B\right) \circ c_{B, B} \circ \delta_{B}, \\
\bar{\omega} \circ\left(B \otimes \bar{\Pi}_{B}^{L}\right) \circ \delta_{B}=\varepsilon_{B}=\bar{\omega} \circ\left(\Pi_{B}^{R} \otimes B\right) \circ c_{B, B} \circ \delta_{B}, \\
\bar{\omega} \circ\left(\Pi_{B}^{L} \otimes B\right) \circ \delta_{B}=\varepsilon_{B}=\bar{\omega} \circ\left(B \otimes \bar{\Pi}_{B}^{R}\right) \circ c_{B, B} \circ \delta_{B}, \\
\omega \circ\left(\Pi_{B}^{R} \otimes B\right)=\omega \circ\left(B \otimes \bar{\Pi}_{B}^{L}\right)=\bar{\omega} \circ\left(B \otimes \Pi_{B}^{L}\right)=\bar{\omega} \circ\left(\bar{\Pi}_{B}^{R} \otimes B\right)=\varepsilon_{B} \circ \mu_{B}, \\
\omega \circ\left(\Pi_{B}^{L} \otimes B\right)=\omega \circ\left(B \otimes \bar{\Pi}_{B}^{R}\right)=\bar{\omega} \circ\left(B \otimes \Pi_{B}^{R}\right)=\bar{\omega} \circ\left(\bar{\Pi}_{B}^{L} \otimes B\right)=\varepsilon_{B} \circ \mu_{B} \circ c_{B, B},
\end{gathered}
$$
hold.

Proof. The proof is the same that the one given for the quasitriangular setting by reversing arrows.

Example 5. Basic examples of quasitriangular weak Hopf monoids are cocommutative weak Hopf monoids because, if $H$ is cocommutative (i.e., $c_{H, H} \circ \delta_{H}=\delta_{H}$ ), the morphisms $\sigma=\bar{\sigma}=\delta_{H} \circ \eta_{H}$ satisfy the conditions of Definition 5. Similarly, commutative weak Hopf monoids (i.e., $\mu_{H} \circ c_{H, H}=$ 
$\mu_{H}$ ) provide examples of coquasitriangular weak Hopf monoids with $\omega=\bar{\omega}=\varepsilon_{H} \circ \mu_{H}$.

The groupoid algebra of a finite groupoid is the main example of a cocommutative weak Hopf monoid. Recall that a finite groupoid $\mathrm{G}$ is simply a category with a finite number of objects in which every morphism is an isomorphism. The set of objects of $\mathrm{G}$ will be denoted by $\mathrm{G}_{0}$, the set of morphisms by $\mathrm{G}_{1}$, the identity morphism on $x \in \mathrm{G}_{0}$ by id $x$ and, for a morphism $g: x \rightarrow y$ in $\mathrm{G}_{1}$, we write $s(g)$ and $t(g)$ for the source and the target of $g$, respectively.

Let $\mathrm{G}$ be a finite groupoid, and let $R$ be a commutative ring. The groupoid algebra is the direct product $R[\mathrm{G}]=\bigoplus_{g \in \mathrm{G}_{1}} R g$ where the product of two morphisms is their composition if the latter is defined and 0 otherwise, i.e., $\mu_{R[G]}\left(g \otimes_{R} h\right)=g \circ h$ if $s(g)=t(h)$ and $\mu_{R[G]}\left(g \otimes_{R} h\right)=0$ if $s(g) \neq t(h)$. The unit element is $1_{R[\mathrm{G}]}=\sum_{x \in \mathrm{G}_{0}} i d_{x}$. The algebra $R[\mathrm{G}]$ is a cocommutative weak Hopf monoid in the symmetric monoidal category R-Mod, with coproduct $\delta_{R[G]}$, counit $\varepsilon_{R[G]}$ and antipode $\lambda_{R[\mathrm{G}]}$ given by $\delta_{R[\mathrm{G}]}(g)=g \otimes_{R} g, \varepsilon_{R[\mathrm{G}]}(g)=1$ and $\lambda_{R[\mathrm{G}]}(g)=g^{-1}$, respectively. Moreover, the target and source morphisms are $\Pi_{R[G]}^{L}(g)=i d_{t(g)}, \Pi_{R[G]}^{R}(g)=i d_{s(g)}$ and the morphism $\sigma$ that provides the quasitriangular structure is the linear extension of

$$
\sigma(1)=\sum_{x \in \mathrm{G}_{0}} i d_{x} \otimes_{R} i d_{x} .
$$

If $\mathrm{G}_{1}$ is finite, $R[\mathrm{G}]$ is free of a finite rank as a $R$-module. Hence $R[\mathrm{G}]$ is finite as object in the category $R$-Mod and $R[\mathrm{G}]^{*}=\operatorname{Hom}_{R}(R[\mathrm{G}], R)=\bigoplus_{g \in \mathrm{G}_{1}} R f_{g}$ is a commutative weak Hopf monoid. The weak Hopf monoid structure of $R[\mathrm{G}]^{*}$ is given by the formulas

$$
\begin{gathered}
1_{R[\mathrm{G}]^{*}}=\sum_{g \in G_{1}} f_{g}, \quad \mu_{H^{*}}\left(f_{g} \otimes_{R} f_{h}\right)=\delta_{g, h} f_{g}, \\
\varepsilon_{R[\mathrm{G}]^{*}}\left(f_{g}\right)=\left\{\begin{array}{ccc}
1 & \text { if } & g=i d_{x} \\
0 & \text { if } & g \neq i d_{x}
\end{array}, \quad \delta_{R[G]^{*}}\left(f_{g}\right)=\sum_{s(g)=s(l)} f_{l} \otimes_{R} f_{g^{\circ} l^{-1}},\right.
\end{gathered}
$$

and

$$
\lambda_{R[\mathrm{G}]^{*}}\left(f_{g}\right)=f_{g^{-1}} .
$$

Then, by the general theory, $R[\mathrm{G}]^{*}$ is an example of coquasitriangular weak Hopf monoid in $R$-Mod where $\omega$ is defined by

$$
\omega\left(\left(f_{g} \otimes_{R} f_{h}\right)\right)=\left\{\begin{array}{ccc}
1 & \text { if } & g=h=i d_{x} \\
0 & & \text { otherwise }
\end{array} .\right.
$$

On the other hand, the construction of a weak Hopf monoid $\mathbb{K}(\mathrm{G}, \mathrm{H})$ in the symmetric monoidal category of vector spaces over a field $\mathbb{K}$ using a matched pair of finite groupoids $(G, H)$ was introduced in [23]. In [24] we can find a result that asserts the following: A matched pair of rotations gives rise to a quasitriangular structure for the associated weak Hopf monoid $\mathbb{K}(G, H)$. Also, by [24] [Theorem 5.10] we know that there is an isomorphism of quasitriangular weak Hopf monoids between the Drinfeld double of $\mathbb{K}(\mathrm{G}, \mathrm{H})$ and the weak Hopf monoid of a suitable matched pair of groupoids.

Finally, in [22], for a weak Hopf monoid $H$ in the symmetric monoidal category of vector spaces over an algebraically closed field, Nikshych, Turaev and Vainerman defined the Drinfeld double $D(H)$ of $H$ and they proved that $D(H)$ is a quasitriangular weak Hopf monoid (see [22] [Proposition 6.2]).

Now we recall the notion of left-left Yetter-Drinfeld module in the weak Hopf monoid setting. 
Definition 7. Let $H$ be a weak Hopf monoid. We shall denote by ${ }_{H}^{H} \mathrm{YD}$ the category of left-left Yetter-Drinfeld modules over $H$, i.e., $M=\left(M, \psi_{M}, \gamma_{M}\right)$ is an object in ${ }_{H}^{H} \mathrm{YD}$ if $\left(M, \psi_{M}\right)$ is a left $H$-module, $\left(M, \gamma_{M}\right)$ is a left $H$-comodule and

$(f 1) \quad\left(\mu_{H} \otimes M\right) \circ\left(H \otimes c_{M, H}\right) \circ\left(\left(\gamma_{M} \circ \psi_{M}\right) \otimes H\right) \circ\left(H \otimes c_{H, M}\right) \circ\left(\delta_{H} \otimes M\right)$ $=\left(\mu_{H} \otimes \psi_{M}\right) \circ\left(H \otimes c_{H, H} \otimes M\right) \circ\left(\delta_{H} \otimes \gamma_{M}\right)$.

(f2) $\quad\left(\mu_{H} \otimes \psi_{M}\right) \circ\left(H \otimes c_{H, H} \otimes M\right) \circ\left(\left(\delta_{H} \circ \eta_{H}\right) \otimes \gamma_{M}\right)=\gamma_{M}$.

Let $\left(M, \psi_{M}, \gamma_{M}\right),\left(N, \psi_{N}, \gamma_{N}\right)$ be objects in ${ }_{H}^{H} Y D$. A morphism $f: M \rightarrow N$ in $\mathrm{C}$ is a morphism of left-left Yetter-Drinfeld modules over $H$ if it is a morphism of left H-modules and left H-comodules.

Please note that if $\left(M, \psi_{M}, \gamma_{M}\right)$ is a left-left Yetter-Drinfeld module, (f2) is equivalent to

$$
\left(\left(\varepsilon_{H} \circ \mu_{H}\right) \otimes \psi_{M}\right) \circ\left(H \otimes c_{H, H} \otimes M\right) \circ\left(\delta_{H} \otimes \gamma_{M}\right)=\psi_{M}
$$

and we have the following identity:

$$
\psi_{M} \circ\left(\Pi_{H}^{L} \otimes M\right) \circ \gamma_{M}=i d_{M}
$$

The conditions (f1) and (f2) of the last definition can also be restated (see [11] [Proposition 2.2]) in the following way: suppose that $\left(M, \psi_{M}\right)$ is a left $H$-module and $\left(M, \gamma_{M}\right)$ is a right H-comodule, then, $\left(M, \psi_{M}, \gamma_{M}\right)$ is in ${ }_{H}^{H} Y D$ if and only if

$$
\begin{gathered}
\gamma_{M} \circ \psi_{M}=\left(\mu_{H} \otimes M\right) \circ\left(H \otimes c_{M, H}\right) \circ \\
\left(\left(\left(\mu_{H} \otimes \psi_{M}\right) \circ\left(H \otimes c_{H, H} \otimes M\right) \circ\left(\delta_{H} \otimes \gamma_{M}\right)\right) \otimes \lambda_{H}\right) \circ\left(H \otimes c_{H, M}\right) \circ\left(\delta_{H} \otimes M\right) .
\end{gathered}
$$

It is a well-known fact that if the antipode of $H$ is an isomorphism, the category ${ }_{H}^{H} Y D$ is a non-strict braided monoidal category. We expose briefly its braided monoidal structure.

For a pair of left-left Yetter-Drinfeld modules over $H\left(M, \psi_{M}, \gamma_{M}\right)$ and $\left(N, \psi_{N}, \gamma_{N}\right)$, there exist two idempotent morphisms $\nabla_{M \otimes N}$ and $\nabla_{M \otimes N}^{\prime}$ defined as in (32) and (40) (for $H=B$ ) respectively. By (iii) of [25] [Proposition 1.12] we have that

$$
\nabla_{M \otimes N}=\nabla_{M \otimes N}^{\prime}
$$

Then, the tensor product in ${ }_{H}^{H} Y D$ for $\left(M, \psi_{M}, \gamma_{M}\right)$ and $\left(N, \psi_{N}, \gamma_{N}\right)$ is introduced as the image of the idempotent morphism $\bar{\nabla}_{M \otimes N}$, denoted by $M \boxminus N$. The object $M \boxminus N$ is a left-left Yetter-Drinfeld module over $H$ with the following action and coaction:

$$
\psi_{M \boxminus N}=p_{M \otimes N} \circ \psi_{M \otimes N} \circ\left(H \otimes i_{M \otimes N}\right), \quad \gamma_{M \boxminus N}=\left(H \otimes p_{M \otimes N}\right) \circ \gamma_{M \otimes N} \circ i_{M \otimes N} .
$$

The base object is $H_{L}$, which is a left-left Yetter-Drinfeld module over $H$ with (co)module structure

$$
\psi_{H_{L}}=p_{H}^{L} \circ \mu_{H} \circ\left(H \otimes i_{H}^{L}\right), \quad \gamma_{H_{L}}=\left(H \otimes p_{H}^{L}\right) \circ \delta_{H} \circ i_{H}^{L} .
$$

The unit constrains are:

$$
\begin{gathered}
\mathfrak{l}_{M}=\psi_{M} \circ\left(i_{H}^{L} \otimes M\right) \circ i_{H_{L} \otimes M}: H_{L} \times M \rightarrow M, \\
\mathfrak{r}_{M}=\psi_{M} \circ c_{M, H} \circ\left(M \otimes\left(\bar{\Pi}_{H}^{L} \circ i_{H}^{L}\right)\right) \circ i_{M \otimes H_{L}}: M \times H_{L} \rightarrow M
\end{gathered}
$$

and the associativity constrains are defined by

$$
\begin{gathered}
\mathfrak{a}_{M, N, P}=p_{M \otimes(N \boxminus P)} \circ\left(M \otimes p_{N \otimes P}\right) \circ\left(i_{M \otimes N} \otimes P\right) \circ i_{(M \boxminus N) \otimes P} \\
:(M \boxminus N) \boxminus P \rightarrow M \boxminus(N \boxminus P) .
\end{gathered}
$$


If $f: M \rightarrow M^{\prime}$ and $g: N \rightarrow N^{\prime}$ are morphisms in the category of left-left Yetter-Drinfeld modules over $H$,

$$
f \boxminus g=p_{M^{\prime} \varpi N^{\prime}} \circ(f \otimes g) \circ i_{M \otimes N}: M \triangleright N \rightarrow M^{\prime} \odot N^{\prime}
$$

is a morphism in ${ }_{H}^{H} \mathrm{YD}$ and

$$
\left(f^{\prime} \bullet g^{\prime}\right) \circ(f \boxminus g)=\left(f^{\prime} \circ f\right) \bullet\left(g^{\prime} \circ g\right),
$$

where $f^{\prime}: M^{\prime} \rightarrow M^{\prime \prime}$ and $g^{\prime}: N^{\prime} \rightarrow N^{\prime \prime}$ are morphisms in ${ }_{H}^{H} Y D$.

Finally, the braiding is

$$
t_{M, N}=p_{N \otimes M} \circ \tau_{M, N} \circ i_{M \otimes N}: M \boxminus N \rightarrow N \boxminus M,
$$

where

$$
\tau_{M, N}=\left(\psi_{N} \otimes M\right) \circ\left(H \otimes c_{M, N}\right) \circ\left(\gamma_{M} \otimes N\right): M \otimes N \rightarrow N \otimes M .
$$

Now we establish a connection between Long dimodules and Yetter-Drinfeld modules.

Theorem 2. Let $H$ be a quasitriangular weak Hopf monoid with morphism $\sigma: H \otimes H \rightarrow K$ and let $B$ be a coquasitriangular weak Hopf monoid with morphism $\omega: K \rightarrow B \otimes B$. There exists a functor

defined on objects by

$$
L:{ }_{H}^{B} \text { Long } \rightarrow \underset{H \otimes B}{H \otimes B} \text { YD }
$$

$$
L\left(\left(M, \varphi_{M}, \rho_{M}\right)\right)=\left(M, \phi_{M}, \varrho_{M}\right),
$$

where

$$
\phi_{M}=\varphi_{M} \circ\left(H \otimes\left(\omega \circ c_{B, B}\right) \otimes M\right) \circ\left(H \otimes B \otimes \rho_{M}\right), \quad \varrho_{M}=\left(H \otimes\left(\rho_{M} \circ \varphi_{M}\right)\right) \circ\left(\left(c_{H, H} \circ \sigma\right) \otimes M\right),
$$

and by the identity on morphisms. Moreover, the functor $L$ is injective on objects and, consequently, ${ }_{H}^{B}$ Long can be identified with a subcategory of ${ }_{H \otimes B}^{H \otimes B}$ YD.

Proof. We begin by showing that $\left(M, \phi_{M}\right)$ is a left $H \otimes B$-module. Indeed, taking into account that $\left(M, \varphi_{M}\right)$ is a left $H$-module, $\left(M, \rho_{M}\right)$ a left $B$-comodule, the naturality of $c$ and (110), we get that $\psi_{M} \circ\left(\eta_{H \otimes B} \otimes M\right)=i d_{M}$. Moreover,

$$
\begin{aligned}
& \phi_{M} \circ\left(H \otimes B \otimes \phi_{M}\right) \\
= & \varphi_{M} \circ\left(H \otimes\left(\omega \circ c_{B, B}\right) \otimes \varphi_{M}\right) \circ\left(H \otimes B \otimes c_{H, B} \otimes M\right) \\
& \circ\left(H \otimes B \otimes H \otimes\left(\left(\omega \circ c_{B, B}\right) \otimes \rho_{M}\right) \circ\left(B \otimes \rho_{M}\right)\right) \text { (by (44)) } \\
= & \varphi_{M} \circ\left(\left(\mu_{H} \circ\left(H \otimes\left(\omega \circ c_{B, B}\right) \otimes H\right)\right) \otimes M\right) \circ\left(H \otimes B \otimes c_{H, B} \otimes M\right) \\
& \circ\left(H \otimes B \otimes H \otimes\left(\left(\left(\omega \circ c_{B, B}\right) \otimes B \otimes M\right) \circ\left(B \otimes\left(\left(\delta_{B} \otimes M\right) \circ \rho_{M}\right)\right)\right)\right) \text { (by the conditions of left } \\
& H \text {-module and a left } B \text {-comodule for } M) \\
= & \varphi_{M} \circ\left(\mu_{H} \otimes\left((\omega \otimes \omega) \circ\left(B \otimes c_{B, B} \otimes B\right) \circ\left(\delta_{B} \otimes c_{B, B}\right) \circ\left(c_{B, B} \otimes B\right) \circ\left(B \otimes c_{B, B}\right)\right) \otimes M\right) \\
& \left.\circ\left(H \otimes c_{B, H} \otimes B \otimes \rho_{M}\right) \text { (by the naturality of c }\right) \\
= & \varphi_{M} \circ\left(\mu_{H} \otimes\left(\omega \circ\left(B \otimes \mu_{B}\right) \circ\left(c_{B, B} \otimes B\right) \circ\left(B \otimes c_{B, B}\right)\right) \otimes M\right) \circ\left(H \otimes c_{B, H} \otimes B \otimes \rho_{M}\right) \\
& \text { (by (e3) of Definition }) \\
= & \left.\phi_{M} \circ\left(\mu_{H \otimes B} \otimes M\right) \text { (by the naturality of } c\right),
\end{aligned}
$$

and $\left(M, \phi_{M}\right)$ is a left $H \otimes B$-module. In a similar way, we can prove that $\left(M, \varrho_{M}\right)$ is a left $H \otimes B$-comodule. Indeed: By (98), the conditions of left $H$-module and left $B$-comodule for $M$ and the naturality of $c$ we have that $\left(\varepsilon_{H \otimes B} \otimes M\right) \circ \varrho_{M}=i d_{M}$. Also,

$$
\begin{aligned}
& \left(\varrho_{M} \otimes M\right) \circ \varrho_{M} \\
= & \left(H \otimes B \otimes \left(\left(H \otimes B \otimes\left(\varphi_{M} \circ\left(H \otimes \varphi_{M}\right)\right)\right) \circ\left(H \otimes c_{H, B} \otimes H \otimes M\right)\right.\right. \\
& \left.\left.\circ\left(\left(c_{H, H} \circ \sigma\right) \otimes c_{H, B} \otimes M\right)\right)\right) \circ\left(H \otimes c_{H, B} \otimes B \otimes M\right) \circ\left(\left(c_{H, H} \circ \sigma\right) \otimes\left(\left(B \otimes \rho_{M}\right) \circ \rho_{M}\right)\right) \\
= & (H \otimes(44)) \\
= & \left(H \otimes B \otimes H \otimes B \otimes \varphi_{M}\right) \circ\left(H \otimes \left(\left(B \otimes H \otimes B \otimes \mu_{H}\right) \circ\left(B \otimes H \otimes c_{H, B} \otimes H\right)\right.\right.
\end{aligned}
$$


$\left.\left.\circ\left(B \otimes\left(c_{H, H} \circ \sigma\right) \otimes c_{H, B}\right) \circ\left(c_{H, B} \otimes B\right) \circ\left(H \otimes \delta_{B}\right)\right) \otimes M\right) \circ\left(\left(c_{H, H} \circ \sigma\right) \otimes \rho_{M}\right)$

(by the conditions of left $H$-module and a left $B$-comodule for $M$ )

$=\left(H \otimes B \otimes H \otimes B \otimes \varphi_{M}\right) \circ\left(H \otimes c_{H, B} \otimes c_{H, B} \otimes M\right) \circ\left(H \otimes H \otimes c_{H, B} \otimes B \otimes M\right)$

$\circ\left(\left(\left(H \otimes c_{H, H}\right) \circ\left(c_{H, H} \otimes H\right) \circ\left(\mu_{H} \otimes c_{H, H}\right) \circ\left(H \otimes c_{H, H} \otimes H\right)\right.\right.$

$\left.\circ(\sigma \otimes \sigma)) \otimes\left(\left(\delta_{B} \otimes M\right) \circ \rho_{M}\right)\right)$ (by the naturality of $c$ )

$=\left(H \otimes B \otimes H \otimes B \otimes \varphi_{M}\right) \circ\left(H \otimes c_{H, B} \otimes c_{H, B} \otimes M\right) \circ\left(H \otimes H \otimes c_{H, B} \otimes B \otimes M\right)$

$\circ\left(\left(\left(H \otimes c_{H, H}\right) \circ\left(c_{H, H} \otimes H\right) \circ\left(H \otimes \delta_{H}\right) \circ \sigma\right) \otimes\left(\left(\delta_{B} \otimes M\right) \circ \rho_{M}\right)\right)$ (by (d3) of Definition 5)

$=\left(\delta_{H \otimes B} \otimes \varphi_{M}\right) \circ\left(H \otimes c_{H, B} \otimes M\right) \circ\left(\left(c_{H, H} \circ \sigma\right) \otimes \rho_{M}\right)$ (by the naturality of $c$ )

$=\left(\delta_{H \otimes B} \otimes M\right) \circ \varrho_{M}$ (by (44)).

To get (f1) of Definition 7, note that on one hand,

$\left(\mu_{H \otimes B} \otimes \phi_{M}\right) \circ\left(H \otimes B \otimes c_{H \otimes B, H \otimes B} \otimes M\right) \circ\left(\delta_{H \otimes B} \otimes Q_{M}\right)$

$=\left(\mu_{H} \otimes B \otimes\left(\varphi_{M} \circ\left(\mu_{H} \otimes M\right)\right)\right) \circ\left(H \otimes H \otimes c_{H, B} \otimes H \otimes M\right)$

$\circ\left(H \otimes c_{H, H} \otimes\left(\left(\mu_{B} \otimes\left(\omega \circ c_{B, B}\right)\right) \circ \delta_{B \otimes B}\right) \otimes H \otimes M\right) \circ\left(\delta_{H} \otimes c_{B, H} \otimes c_{H, B} \otimes M\right)$

$\circ\left(H \otimes B \otimes\left(c_{H, H} \circ \sigma\right) \otimes \rho_{M}\right)$ (by (44), the conditions of left $H$-module and a left $B$-comodule for $M$ and the naturality of $c$ )

$=\left(\mu_{H} \otimes B \otimes\left(\varphi_{M} \circ\left(\mu_{H} \otimes M\right)\right)\right) \circ\left(H \otimes H \otimes c_{H, B} \otimes H \otimes M\right)$

$\circ\left(H \otimes c_{H, H} \otimes\left(\left(\left(\mu_{B} \circ c_{B, B}\right) \otimes \omega\right) \circ \delta_{B \otimes B} \circ c_{B, B}\right) \otimes H \otimes M\right) \circ\left(\delta_{H} \otimes c_{B, H} \otimes c_{H, B} \otimes M\right)$

$\circ\left(H \otimes B \otimes\left(c_{H, H} \circ \sigma\right) \otimes \rho_{M}\right)$ (by the naturality of $\left.c\right)$

$=\left(\mu_{H} \otimes B \otimes\left(\varphi_{M} \circ\left(\mu_{H} \otimes M\right)\right)\right) \circ\left(H \otimes H \otimes c_{H, B} \otimes H \otimes M\right) \circ\left(H \otimes c_{H, H} \otimes\left(\left(\omega \otimes \mu_{B}\right)\right.\right.$ $\left.\left.\circ \delta_{B \otimes B} \circ c_{B, B}\right) \otimes H \otimes M\right) \circ\left(\delta_{H} \otimes c_{B, H} \otimes c_{H, B} \otimes M\right) \circ\left(H \otimes B \otimes\left(c_{H, H} \circ \sigma\right) \otimes \rho_{M}\right)$ (by (e4) of Definition 6)

$=\left(H \otimes\left(\left(\left(\left(\omega \otimes \mu_{B}\right) \circ \delta_{B \otimes B}\right) \otimes \varphi_{M}\right) \circ\left(B \otimes c_{H, B} \otimes M\right) \circ\left(c_{H, B} \otimes B \otimes M\right)\right)\right)$ $\circ\left(\left(\mu_{H \otimes H} \circ\left(\delta_{H} \otimes\left(c_{H, H} \circ \sigma\right)\right)\right) \otimes\left(\left(c_{B, B} \otimes M\right) \circ\left(B \otimes \rho_{M}\right)\right)\right)$

(by the naturality of $c$ )

$\left.=\left(H \otimes\left(\left(\left(\omega \otimes \mu_{B}\right) \circ \delta_{B \otimes B}\right) \otimes \varphi_{M}\right) \circ\left(B \otimes c_{H, B} \otimes M\right) \circ\left(c_{H, B} \otimes B \otimes M\right)\right)\right)$

$\circ\left(\left(c_{H, H} \circ \mu_{H \otimes H} \circ\left(\left(c_{H, H} \circ \delta_{H}\right) \otimes \sigma\right)\right) \otimes\left(\left(c_{B, B} \otimes M\right) \circ\left(B \otimes \rho_{M}\right)\right)\right)$ (by the naturality of $\left.c\right)$

$=\left(H \otimes\left(\left(\left(\left(\omega \otimes \mu_{B}\right) \circ \delta_{B \otimes B}\right) \otimes \varphi_{M}\right) \circ\left(B \otimes c_{H, B} \otimes M\right) \circ\left(c_{H, B} \otimes B \otimes M\right)\right)\right)$

$\circ\left(\left(c_{H, H} \circ \mu_{H \otimes H} \circ\left(\sigma \otimes \delta_{H}\right)\right) \otimes\left(\left(c_{B, B} \otimes M\right) \circ\left(B \otimes \rho_{M}\right)\right)\right)$ (by (d4) of Definition 5)

$=\left(H \otimes B \otimes \varphi_{M}\right) \circ\left(H \otimes c_{H, B} \otimes M\right) \circ\left(\left(c_{H, H} \circ\left(\mu_{H \otimes H} \circ\left(\sigma \otimes \delta_{H}\right)\right)\right) \otimes\left(\left(\omega \otimes \mu_{B}\right)\right.\right.$ $\left.\left.\circ \delta_{B \otimes B} \circ \mathcal{c}_{B, B}\right) \otimes M\right) \circ\left(H \otimes B \otimes \rho_{M}\right)$ (by the naturality of $c$ ),

and on the other hand,

$\left(\mu_{H \otimes B} \otimes M\right) \circ\left(H \otimes B \otimes c_{M, H \otimes B}\right) \circ\left(\left(\varrho_{M} \circ \phi_{M}\right) \otimes H \otimes B\right) \circ\left(H \otimes B \otimes c_{H \otimes B, M}\right)$ $\circ\left(\delta_{H \otimes B} \otimes M\right)$

$=\left(\mu_{H} \otimes \mu_{B} \otimes M\right) \circ\left(H \otimes c_{B, H} \otimes c_{M, H}\right) \circ\left(H \otimes B \otimes c_{M, H} \otimes B\right) \circ\left(H \otimes\left(\left(B \otimes \varphi_{M}\right)\right.\right.$ $\circ\left(c_{H, B} \otimes \varphi_{M}\right) \circ\left(H \otimes c_{H, B} \otimes M\right) \circ\left(H \otimes H \otimes\left(\left(\left(\omega \circ c_{B, B}\right) \otimes \rho_{M}\right)\right.\right.$

$\left.\left.\circ\left(B \otimes \rho_{M}\right)\right) \otimes H \otimes B\right) \circ\left(\left(c_{H, H} \circ \sigma\right) \otimes\left(\left(H \otimes B \otimes c_{H, M} \otimes B\right) \circ\left(H \otimes c_{H . B} \otimes c_{B, M}\right)\right.\right.$ $\left.\left.\circ\left(\delta_{H} \otimes \delta_{B} \otimes M\right)\right)\right)($ by (44))

$=\left(H \otimes B \otimes \varphi_{M}\right) \circ\left(H \otimes c_{H, B} \otimes M\right) \circ\left(\left(\left(\mu_{H} \otimes H\right) \circ\left(H \otimes c_{H, H}\right) \otimes\left(H \otimes \mu_{H} \otimes H\right)\right.\right.$ $\left.\left.\circ\left(\left(c_{H, H} \circ \sigma\right) \otimes \delta_{H}\right)\right) \otimes\left(\left(\left(\omega \circ c_{B, B}\right) \otimes \mu_{B}\right) \circ\left(B \otimes \delta_{B} \otimes B\right) \circ\left(B \otimes c_{B, B}\right) \circ\left(\delta_{B} \otimes B\right)\right) \otimes M\right)$ $\circ\left(H \otimes B \otimes \rho_{M}\right)$ (by the conditions of left $H$-module and a left $B$-comodule for $M$ and by the naturality of $c$ )

$=\left(H \otimes B \otimes \varphi_{M}\right) \circ\left(H \otimes c_{H, B} \otimes M\right) \circ\left(\left(c_{H, H} \circ\left(\mu_{H \otimes H} \circ\left(\sigma \otimes \delta_{H}\right)\right)\right) \otimes\left(\left(\omega \otimes \mu_{B}\right) \circ \delta_{B \otimes B}\right.\right.$ $\left.\left.\circ \mathcal{C}_{B, B}\right) \otimes M\right) \circ\left(H \otimes B \otimes \rho_{M}\right)$ (by the naturality of $c$ ),

The condition (f2) of Definition 7 follows because, using the previous calculus,

$\left(\mu_{H \otimes B} \otimes \phi_{M}\right) \circ\left(H \otimes B \otimes c_{H \otimes B, H \otimes B} \otimes M\right) \circ\left(\delta_{H \otimes B} \otimes \varrho_{M}\right) \circ\left(\eta_{H} \otimes \eta_{B} \otimes M\right)$

$=\left(H \otimes B \otimes \varphi_{M}\right) \circ\left(H \otimes c_{H, B} \otimes M\right) \circ\left(\left(c_{H, H} \circ\left(\mu_{H \otimes H} \circ\left(\sigma \otimes\left(\delta_{H} \circ \eta_{H}\right)\right)\right)\right) \otimes\left(\left(\omega \otimes \mu_{B}\right)\right.\right.$ $\left.\left.\circ \delta_{B \otimes B} \circ c_{B, B}\right) \otimes M\right) \circ\left(H \otimes \eta_{B} \otimes \rho_{M}\right)$ (by identity obtained to prove (f1) of Definition 7)

$=\left(H \otimes B \otimes \varphi_{M}\right) \circ\left(\left(c_{H, H} \circ \Omega_{H}^{2} \circ \sigma\right) \otimes\left(\left(\left(\omega \circ\left(B \otimes \Pi_{B}^{R}\right) \circ \delta_{B}\right) \otimes B\right) \circ \delta_{B}\right) \otimes M\right) \circ \rho_{M}$ (by (12) for $B$ and $H$ and coassociativity of $\delta_{B}$ )

$=\left(H \otimes B \otimes \varphi_{M}\right) \circ\left(H \otimes c_{H, B} \otimes M\right) \circ\left(\left(c_{H, H} \circ \sigma\right) \otimes \rho_{M}\right)$ (by (112), (d1) of Definition 5, the properties of $\varepsilon_{B}$ and (84))

$=\varrho_{M}($ by (44)). 
Finally, if $f:\left(M, \varphi_{M}, \rho_{M}\right) \rightarrow\left(N, \varphi_{N}, \rho_{N}\right)$ is a morphism in ${ }_{H}^{B}$ Long, by the left $H$ linearity and the left $B$-coliniarity, we have that $f:\left(M, \phi_{M}, \varrho_{M}\right) \rightarrow\left(N, \phi_{M}, \varrho_{N}\right)$ is a morphism in the category ${ }_{H \otimes B}^{H \otimes B}$ YD.

As a consequence of the previous facts, there exists a functor

$$
L: \stackrel{B}{H} \text { Long } \rightarrow{ }_{H \otimes B}^{H \otimes B} Y \mathrm{D}
$$

defined on objects by

$$
L\left(\left(M, \varphi_{M}, \rho_{M}\right)\right)=\left(M, \phi_{M}, \varrho_{M}\right),
$$

and by the identity on morphisms. Finally, if $L\left(\left(M, \varphi_{M}, \rho_{M}\right)\right)=L\left(\left(N, \varphi_{N}, \rho_{N}\right)\right)$, it is obvious that $M=N$ and, by (110) and the condition of left $B$-comodule for $M$, we have

$$
\varphi_{M}=\phi_{M} \circ\left(H \otimes \eta_{B} \otimes M\right)=\phi_{N} \circ\left(H \otimes \eta_{B} \otimes N\right)=\varphi_{N}
$$

Similarly, by (98) and the condition of left $H$-module for $M$, we obtain

$$
\rho_{M}=\left(\varepsilon_{H} \otimes B \otimes M\right) \circ \varrho_{M}=\left(\varepsilon_{H} \otimes B \otimes M\right) \circ \varrho_{N}=\rho_{N} .
$$

Thus, $L$ is injective on objects.

Lemma 9. Let $H$ be a quasitriangular weak Hopf monoid with morphism $\sigma: H \otimes H \rightarrow K$ and let $B$ be a coquasitriangular weak Hopf monoid with morphism $\omega: K \rightarrow B \otimes B$. Let L be the functor introduced in Theorem 2. Then, for all $\left(M, \varphi_{M}, \rho_{M}\right)$ and $\left(N, \varphi_{N}, \rho_{N}\right)$ in ${ }_{H}^{B}$ Long,

$$
\operatorname{Hom}_{H} \text { Long }\left(\left(M, \varphi_{M}, \rho_{M}\right),\left(N, \varphi_{N}, \rho_{N}\right)\right)=\operatorname{Hom}_{H \otimes B}^{H \otimes B}\left(L\left(\left(M, \varphi_{M}, \rho_{M}\right)\right), L\left(\left(N, \varphi_{N}, \rho_{N}\right)\right)\right) \text {. }
$$

Proof. By Theorem 2 we know that

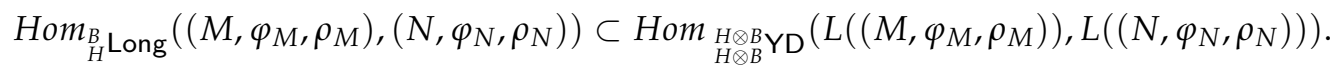

On the other hand, let $g: L\left(\left(M, \varphi_{M}, \rho_{M}\right)\right) \rightarrow L\left(\left(N, \varphi_{N}, \rho_{N}\right)\right)$ be a morphism in $H \otimes B$ YD. Then, $g$ is a morphism of left $H \otimes B$-modules, i.e., $g \circ \phi_{M}=\phi_{N} \circ(H \otimes B \otimes g)$. Composing in this equality with $H \otimes \eta_{B} \otimes M$, by (110) and the condition of left $B$-comodule for $M$, we have that $g \circ \varphi_{M}=\varphi_{N} \circ(H \otimes g)$. Therefore, $g$ is a morphism of lef $H$-modules. On the other hand, $g$ is a morphism of left $H \otimes B$-comodules, i.e., $(H \otimes B \otimes g) \circ \varrho_{M}=\varrho_{N} \circ g$. Composing in this equality with $\varepsilon_{H} \otimes B \otimes M$, by (98) and the condition of left $H$-module for $M$, we have that $(B \otimes g) \circ \rho_{M}=\rho_{N} \circ g$. Thus, $g$ is a morphism of lef $B$-comodules. Consequently, we can assure that $g$ is a morphism in ${ }_{H}^{B}$ Long between $\left(M, \varphi_{M}, \rho_{M}\right)$ and $\left(N, \varphi_{N}, \rho_{N}\right)$ and then,

$$
\operatorname{Hom}_{\substack{H \otimes B \\ \otimes B D}}\left(L\left(\left(M, \varphi_{M}, \rho_{M}\right)\right), L\left(\left(N, \varphi_{N}, \rho_{N}\right)\right)\right) \subset \operatorname{Hom}_{H} \operatorname{Long}\left(\left(M, \varphi_{M}, \rho_{M}\right),\left(N, \varphi_{N}, \rho_{N}\right)\right) .
$$

Lemma 10. Let $H$ be a quasitriangular weak Hopf monoid with morphism $\sigma: H \otimes H \rightarrow K$ and let $B$ be a coquasitriangular weak Hopf monoid with morphism $\omega: K \rightarrow B \otimes B$. Let L be the functor introduced in Theorem 2. Then,

$$
L\left(\left(H_{L} \otimes B_{L}, \varphi_{H_{L} \otimes B_{L}}, \rho_{H_{L} \otimes B_{L}}\right)\right)=\left(H_{L} \otimes B_{L}, \psi_{H_{L} \otimes B_{L}}, \gamma_{H_{L} \otimes B_{L}}\right),
$$

where $\psi_{H_{L} \otimes B_{L}}$ and $\gamma_{H_{L} \otimes B_{L}}$ are the action and the coaction introduced in (122).

Proof. To prove the Lemma, by the naturality of $c$, we only need to show that the equalities

$$
\left(\left(\omega \circ c_{B, B}\right) \otimes p_{B}^{L}\right) \circ\left(B \otimes\left(\delta_{B} \circ i_{B}^{L}\right)\right)=p_{B}^{L} \circ \mu_{B} \circ\left(B \otimes i_{B}^{L}\right)
$$


and

$$
\left(H \otimes\left(p_{H}^{L} \circ \mu_{H}\right)\right) \circ\left(\left(c_{H, H} \circ \sigma\right) \otimes i_{H}^{L}\right)=\left(H \otimes p_{H}^{L}\right) \circ \delta_{H} \circ i_{H}^{L}
$$

hold. Indeed: On one hand

$$
\begin{aligned}
& \left(\left(\omega \circ c_{B, B}\right) \otimes p_{B}^{L}\right) \circ\left(B \otimes\left(\delta_{B} \circ i_{B}^{L}\right)\right) \\
= & \left.\left(\left(\omega \circ c_{B, B}\right) \otimes p_{B}^{L}\right) \circ\left(B \otimes\left(\left(\left(B \otimes \Pi_{B}^{L}\right) \circ \delta_{B}\right) \circ i_{B}^{L}\right)\right) \text { (by the idempotent condition for } \Pi_{B}^{L}\right) \\
= & \left(\left(\omega \circ c_{B, B} \circ\left(B \otimes \mu_{B}\right)\right) \otimes p_{B}^{L}\right) \circ\left(B \otimes\left(\left(B \otimes c_{B, B}\right) \circ\left(\left(\delta_{B} \circ \eta_{B}\right) \otimes i_{B}^{L}\right)\right)\right) \text { (by (10)) } \\
= & \left.\left((\omega \otimes \omega) \circ\left(B \otimes c_{B, B} \otimes B\right) \circ\left(B \otimes B \otimes \delta_{B}\right) \circ\left(B \otimes c_{B, B}\right) \otimes\left(c_{B, B} \otimes B\right)\right) \otimes p_{B}^{L}\right) \\
& \circ\left(B \otimes\left(\left(B \otimes c_{B, B}\right) \circ\left(\left(\delta_{B} \circ \eta_{B}\right) \otimes i_{B}^{L}\right)\right)\right) \text { (by the naturality of c and (e3) of Definition 6) } \\
= & \left(\left(\left(\omega \circ\left(\Pi_{B}^{R} \otimes B\right)\right) \otimes\left(\omega \circ\left(\Pi_{B}^{L} \otimes B\right)\right)\right) \circ\left(B \otimes c_{B, B} \otimes B\right) \circ\left(B \otimes B \otimes \delta_{B}\right)\right. \\
& \left.\left.\circ\left(B \otimes c_{B, B}\right) \otimes\left(c_{B, B} \otimes B\right)\right) \otimes p_{B}^{L}\right) \circ\left(B \otimes\left(\left(B \otimes c_{B, B}\right) \circ\left(\left(\delta_{B} \circ \eta_{B}\right) \otimes i_{B}^{L}\right)\right)\right) \text { (by (17)) } \\
= & \left(\left(\left(\left(\varepsilon_{B} \circ \mu_{B}\right) \otimes\left(\varepsilon_{B} \circ \mu_{B} \circ c_{B, B}\right)\right)\right)\left(B \otimes c_{B, B} \otimes B\right) \circ\left(B \otimes B \otimes \delta_{B}\right)\right. \\
& \left.\left.\circ\left(B \otimes c_{B, B}\right) \otimes\left(c_{B, B} \otimes B\right)\right) \otimes p_{B}^{L}\right) \circ\left(B \otimes\left(\left(B \otimes c_{B, B}\right) \circ\left(\left(\delta_{B} \circ \eta_{B}\right) \otimes i_{B}^{L}\right)\right)\right) \text { (by (115) and (116)) } \\
= & \left(\left(\varepsilon_{B} \circ \mu_{B}\right) \otimes p_{B}^{L} \otimes\left(\varepsilon_{B} \circ \mu_{B}\right)\right) \circ\left(B \otimes c_{B, B} \otimes B \otimes B\right) \otimes\left(\left(\delta_{B} \circ \eta_{B}\right) \otimes \delta_{B} \otimes i_{B}^{L}\right) \\
& (\text { by the naturality pf } c) \\
= & \left.\left.\left(p_{B}^{L} \otimes\left(\varepsilon_{B} \circ \mu_{B}\right)\right) \circ\left(\delta_{B} \otimes i_{B}^{L}\right) \text { (by the idempotent condition for } \Pi_{B}^{L}\right)\right) \\
= & p_{B}^{L} \circ \mu_{B} \circ\left(B \otimes\left(\bar{\Pi}_{B}^{L} \circ i_{B}^{L}\right)\right) \text { (by (14)) } \\
= & p_{B}^{L} \circ \mu_{B} \circ\left(B \otimes\left(\Pi_{B}^{L} \circ \bar{\Pi}_{B}^{L} \circ i_{B}^{L}\right)\right) \text { (by (5)) } \\
= & p_{B}^{L} \circ \mu_{B} \circ\left(B \otimes i_{B}^{L}\right)\left(\text { by (1) and the idempotent condition for } \Pi_{B}^{L}\right),
\end{aligned}
$$

and, on the other hand,

$$
\begin{aligned}
& \left(H \otimes\left(p_{H}^{L} \circ \mu_{H}\right)\right) \circ\left(\left(c_{H, H} \circ \sigma\right) \otimes i_{H}^{L}\right) \\
= & \left(H \otimes\left(p_{H}^{L} \circ \mu_{H}\right)\right) \circ\left(\left(c_{H, H} \circ \sigma\right) \otimes\left(\Pi_{H}^{L} \circ i_{H}^{L}\right)\right)(\text { by (5)) } \\
= & \left(H \otimes\left(\left(\left(\varepsilon_{H} \circ \mu_{H}\right) \otimes p_{H}^{L}\right) \circ\left(H \otimes c_{H, H}\right) \circ\left(\delta_{H} \otimes H\right)\right)\right) \circ\left(\left(c_{H, H} \circ \sigma\right) \otimes i_{H}^{L}\right)(\text { by (9)) } \\
= & \left.\left(H \otimes\left(\varepsilon_{H} \circ \mu_{H}\right) \otimes p_{H}^{L}\right) \circ\left(c_{H, H} \otimes c_{H, H}\right) \circ\left(H \otimes c_{H, H} \otimes H\right) \circ\left(\left(\delta_{H} \otimes H\right) \circ \sigma\right) \otimes i_{H}^{L}\right) \\
& (\text { by the naturality of } c) \\
= & \left(H \otimes\left(\varepsilon_{H} \circ \mu_{H}\right) \otimes p_{H}^{L}\right) \circ\left(c_{H, H} \otimes c_{H, H}\right) \circ\left(H \otimes c_{H, H} \otimes H\right) \circ\left(\left(\left(H \otimes H \otimes \mu_{H}\right)\right.\right. \\
& \left.\left.\circ\left(H \otimes c_{H, H} \otimes H\right) \circ(\sigma \otimes \sigma)\right) \otimes i_{H}^{L}\right)(\text { by (d2) of Definition } 5) \\
= & \left(H \otimes\left(\varepsilon_{H} \circ \mu_{H}\right) \otimes p_{H}^{L}\right) \circ\left(c_{H, H} \otimes c_{H, H}\right) \circ\left(H \otimes c_{H, H} \otimes H\right) \circ\left(\left(\left(H \otimes H \otimes \mu_{H}\right)\right.\right. \\
& \left.\left.\circ\left(H \otimes c_{H, H} \otimes H\right) \circ\left(\left(\left(\Pi \Pi_{H}^{R} \otimes H\right) \circ \sigma\right) \otimes\left(\left(\Pi \Pi_{H}^{L} \otimes H\right) \circ \sigma\right)\right)\right) \otimes i_{H}^{L}\right) \\
& \left(\text { by }(18), \text { the idempotent condition for } \Pi_{H}^{L} \text { and the naturality of } c\right) \\
= & \left(H \otimes\left(\varepsilon_{H} \circ \mu_{H}\right) \otimes p_{H}^{L}\right) \circ\left(c_{H, H} \otimes c_{H, H}\right) \circ\left(H \otimes c_{H, H} \otimes H\right) \circ\left(\left(\left(H \otimes H \otimes \mu_{H}\right)\right.\right. \\
& \left.\circ\left(H \otimes c_{H, H} \otimes H\right) \circ\left(\left(\delta_{H} \circ \eta_{H}\right) \otimes\left(c_{H, H} \circ \delta_{H} \circ \eta_{H}\right)\right) \otimes i_{H}^{L}\right)((103) \text { and (104)) } \\
= & \left(H \otimes\left(\varepsilon_{H} \circ \mu_{H}\right) \otimes p_{H}^{L}\right) \circ\left(c_{H, H} \otimes c_{H, H}\right) \circ\left(\left(\left(H \otimes \mu_{H} \otimes H\right)\right.\right. \\
& \left.\left.\circ\left(\left(\delta_{H} \circ \eta_{H}\right) \otimes\left(\delta_{H} \circ \eta_{H}\right)\right)\right) \otimes i_{H}^{L}\right)(\text { by the naturality of } c) \\
= & \left(\left(\left(\left(\varepsilon_{H} \circ \mu_{H}\right) \otimes H\right) \circ\left(H \otimes c_{H, H}\right) \circ\left(\delta_{H} \otimes H\right)\right) \otimes p_{H}^{L}\right) \circ\left(H \otimes c_{H, H} \otimes H\right) \\
& \circ\left(\left(\delta_{H} \circ \eta_{H}\right) \otimes i_{H}^{L}\right)(\text { by (a3) of Definition } 1 \text { and naturality of } c) \\
= & \left(\left(\mu_{H} \circ\left(H \otimes \Pi_{H}^{L}\right)\right) \otimes p_{H}^{L}\right) \circ\left(H \otimes c_{H, H} \otimes H\right) \circ\left(\left(\delta_{H} \circ \eta_{H}\right) \otimes i_{H}^{L}\right)(\text { by (9)) } \\
= & \left(\mu_{H} \otimes p_{H}^{L}\right) \circ\left(H \otimes c_{H, H} \otimes H\right) \circ\left(\left(\delta_{H} \circ \eta_{H}\right) \otimes i_{H}^{L}\right)\left(\text { by the idempotent condition for } \Pi_{H}^{L}\right) \\
= & \left(H \otimes\left(p_{H}^{L} \circ \Pi_{H}^{L}\right)\right) \circ \delta_{H} \circ i_{H}^{L}(\text { by }(10)) \\
= & \left(H \otimes p_{H}^{L}\right) \circ \delta_{H} \circ i_{H}^{L}\left(\text { by the idempotent condition for } \Pi_{H}^{L}\right) .
\end{aligned}
$$

Lemma 11. Let $H$ be a quasitriangular weak Hopf monoid with morphism $\sigma: H \otimes H \rightarrow K$ and let $B$ be a coquasitriangular weak Hopf monoid with morphism $\omega: K \rightarrow B \otimes B$. Let $L$ be the functor introduced in Theorem 2. Let $\left(M, \varphi_{M}, \rho_{M}\right)$ and $\left(N, \varphi_{N}, \rho_{N}\right)$ be objects in ${ }_{H}^{B}$ Long and let $\left(M, \phi_{M}, \varrho_{M}\right)$ and $\left(N, \phi_{N}, \varrho_{N}\right)$ are their corresponding images in ${ }_{H \otimes B}^{H \otimes B} Y \mathrm{Y}$ by the functor $L$. The following equality

$$
\nabla_{M \otimes N}=\Omega_{M \otimes N}
$$

holds. As a consequence, $M \times N=M \boxminus N$ and, if $\left(P, \varphi_{P}, \rho_{P}\right)$ is another object in ${ }_{H}^{B}$ Long, we have that

$$
a_{M, N, P}=\mathfrak{a}_{M, N, P},
$$


where $a_{M, N, P}$ is the associative constraint introduced in Proposition 2 and $\mathfrak{a}_{M, N, P}$ the corresponding one for $\left(M, \phi_{M}, \varrho_{M}\right),\left(N, \phi_{N}, \varrho_{N}\right)$ and $\left(P, \phi_{P}, \varrho_{P}\right)$ in ${ }_{H \otimes B}^{H \otimes B} \mathrm{YD}$.

Proof. Let $\left(M, \varphi_{M}, \rho_{M}\right)$ and $\left(N, \varphi_{N}, \rho_{N}\right)$ be objects in ${ }_{H}^{B}$ Long and let $\left(M, \phi_{M}, \gamma_{M}\right)$ and $\left(N, \phi_{N}, \gamma_{N}\right)$ are their corresponding images in ${ }_{H \otimes B}^{H \otimes B} Y$ D by the functor $L$. Then,

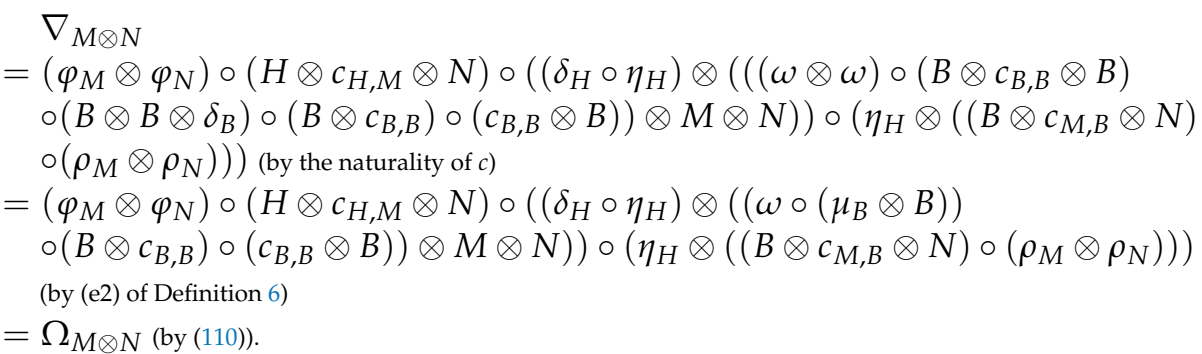

Finally, the equality for the associative constraints follows (125) and Proposition 2.

Lemma 12. Let $H$ be a quasitriangular weak Hopf monoid with morphism $\sigma: H \otimes H \rightarrow K$ and let $B$ be a coquasitriangular weak Hopf monoid with morphism $\omega: K \rightarrow B \otimes B$. Let $L$ be the functor introduced in Theorem 2. Let $\left(M, \varphi_{M}, \rho_{M}\right)$ be in ${ }_{H}^{B}$ Long and let $\left(M, \phi_{M}, \varrho_{M}\right)$ its corresponding image in $\begin{gathered}H \otimes B \\ H \otimes B\end{gathered}$ YD by the functor $L$. Then,

(i) If $l_{M}$ is the left unit constraint introduced in Proposition 3 for $\left(M, \varphi_{M}, \rho_{M}\right)$ and $\mathfrak{l}_{M}$ is the corresponding unit constraint defined in (123) for $\left(M, \phi_{M}, \varrho_{M}\right)$, we have that $l_{M}=\mathfrak{l}_{M}$.

(ii) If $r_{M}$ is the right unit constraint introduced in Proposition 3 for $\left(M, \varphi_{M}, \rho_{M}\right)$ and $\mathfrak{r}_{M}$ is the corresponding unit constraint defined in (124) for $\left(M, \phi_{M}, \varrho_{M}\right)$, we have that $r_{M}=\mathfrak{r}_{M}$.

Proof. $\mathrm{t}\left(M, \varphi_{M}, \rho_{M}\right)$ be in ${ }_{H}^{B}$ Long and let $\left(M, \phi_{M}, \varrho_{M}\right)$ its corresponding image in ${ }_{H \otimes B}^{H \otimes B}$ YD by the functor $L$. First note that by the previous lemma, we have the following identities:

$$
\begin{gathered}
\left(H_{L} \otimes B_{L}\right) \times M=\left(H_{L} \otimes B_{L}\right) \bullet M, \quad M \times\left(H_{L} \otimes B_{L}\right)=M \otimes\left(H_{L} \otimes B_{L}\right), \\
p_{\left(H_{L} \otimes B_{L}\right) \otimes M}=q_{\left(H_{L} \otimes B_{L}\right) \otimes M}, \quad i_{\left(H_{L} \otimes B_{L}\right) \otimes M}=j_{\left(H_{L} \otimes B_{L}\right) \otimes M}
\end{gathered}
$$

and

$$
p_{M \otimes\left(H_{L} \otimes B_{L}\right)}=q_{M \otimes\left(H_{L} \otimes B_{L}\right)}, \quad i_{M \otimes\left(H_{L} \otimes B_{L}\right)}=j_{M \otimes\left(H_{L} \otimes B_{L}\right)} .
$$

Then,

$$
\begin{aligned}
& \mathfrak{l}_{M} \\
= & \varphi_{M} \circ\left(H \otimes\left(\omega \circ c_{B, B}\right) \otimes M\right) \circ\left(i_{H}^{L} \otimes i_{B}^{L} \otimes \rho_{M}\right) \circ i_{\left(H_{L} \otimes B_{L}\right) \otimes M}(\text { by (123)) } \\
= & \varphi_{M} \circ\left(H \otimes\left(\omega \circ\left(B \otimes \bar{\Pi}_{B}^{R}\right) \circ c_{B, B}\right) \otimes M\right) \circ\left(i_{H}^{L} \otimes i_{B}^{L} \otimes \rho_{M}\right) \circ i_{\left(H_{L} \otimes B_{L}\right) \otimes M}(\text { by (2)) } \\
= & \varphi_{M} \circ\left(H \otimes\left(\varepsilon_{B} \circ \mu_{B}\right) \otimes M\right) \circ\left(i_{H}^{L} \otimes i_{B}^{L} \otimes \rho_{M}\right) \circ i_{\left(H_{L} \otimes B_{L}\right) \otimes M}(\text { by (116)) } \\
= & \left(\left(\varepsilon_{B} \circ \mu_{B}\right) \otimes \varphi_{M}\right) \circ\left(B \otimes c_{B, H} \otimes M\right) \circ\left(\left(c_{H, B} \circ\left(i_{H}^{L} \otimes i_{B}^{L}\right)\right) \otimes \rho_{M}\right) \circ i_{\left(H_{L} \otimes B_{L}\right) \otimes M} \\
& (\text { by the naturality of } c) \\
= & l_{M} \text { (by (44)) }
\end{aligned}
$$

and

$$
\begin{aligned}
& \mathfrak{r}_{M} \\
= & \varphi_{M} \circ\left(H \otimes\left(\omega \circ\left(B \otimes \bar{\Pi}_{B}^{L}\right) \circ c_{B, B}\right) \otimes M\right) \circ\left(H \otimes B \otimes \rho_{M}\right) \circ\left(H \otimes c_{M, B}\right) \circ\left(c_{M, H} \otimes B\right) \\
& \left.\circ\left(M \otimes\left(\bar{\Pi}_{H}^{L} \circ i_{H}^{L}\right) \otimes i_{B}^{L}\right) \circ i_{M \otimes\left(H_{L} \otimes B_{L}\right)} \text { (by (124) and the naturality of } c\right) \\
= & \varphi_{M} \circ\left(H \otimes\left(\varepsilon_{B} \circ \mu_{B} \circ c_{B, B}\right) \otimes M\right) \circ\left(H \otimes B \otimes \rho_{M}\right) \circ\left(H \otimes c_{M, B}\right) \circ\left(c_{M, H} \otimes B\right) \\
& \circ\left(M \otimes\left(\bar{\Pi}_{H}^{L} \circ i_{H}^{L}\right) \otimes i_{B}^{L}\right) \circ i_{M \otimes\left(H_{L} \otimes B_{L}\right)}(\text { by (115)) } \\
= & \left.r_{M} \text { (by the naturality of } c\right) .
\end{aligned}
$$


In the final result of this paper, we will prove that under the conditions of the previous theorem, ${ }_{H}^{B}$ Long is a braided monoidal category.

Theorem 3. Let $H$ be a quasitriangular weak Hopf monoid with morphism $\sigma: H \otimes H \rightarrow K$ and let $B$ be a coquasitriangular weak Hopf monoid with morphism $\omega: K \rightarrow B \otimes B$. Then, the category ${ }_{H}^{B}$ Long is a braided monoidal. Furthermore, if $H$ is triangular and $B$ is cotriangular, then, ${ }_{H}^{B}$ Long is symmetric.

Proof. The main assertion of this theorem is a direct consequence of the preceding Lemmas. Please note that the braiding in ${ }_{H}^{B}$ Long is the one defined in (128) for the category ${ }_{H}^{H \otimes B} \otimes B$ Y. Therefore, if $\left(M, \varphi_{M}, \rho_{M}\right)$ and $\left(N, \varphi_{N}, \rho_{N}\right)$ are objects in ${ }_{H}^{B}$ Long, by (44) and the naturality of $c$, the braiding admits the following formulation:

$t_{M, N}=\left(\omega \otimes p_{N \otimes M}\right) \circ\left(B \otimes c_{N, B} \otimes M\right) \circ\left(\rho_{M} \otimes \rho_{N}\right) \circ c_{M, N} \circ\left(\varphi_{M} \otimes \varphi_{N}\right) \circ\left(H \otimes c_{H, M} \otimes N\right) \circ\left(\sigma \otimes i_{M \otimes N}\right)$.

On the other hand, if $H$ is triangular and $B$ is cotriangular, i.e., $\bar{\sigma}=c_{H, H} \circ \sigma$ and $\bar{\omega}=\omega \circ c_{B, B}$, we have that:

$$
\begin{aligned}
& t_{N, M} \circ t_{M, N} \\
= & \left(\omega \otimes p_{M \otimes N}\right) \circ\left(B \otimes c_{M, B} \otimes N\right) \circ\left(\rho_{M} \otimes \rho_{N}\right) \circ c_{N, M} \circ\left(\varphi_{N} \otimes \varphi_{M}\right) \circ\left(H \otimes c_{H, N} \otimes M\right) \\
& \circ(\sigma \otimes N \otimes M) \circ\left(\omega \otimes \nabla_{N \otimes M} \circ\left(B \otimes c_{N, B} \otimes M\right) \circ\left(\rho_{N} \otimes \rho_{M}\right) \circ c_{M, N} \circ\left(\varphi_{M} \otimes \varphi_{N}\right)\right. \\
& \circ\left(H \otimes c_{H, M} \otimes N\right) \circ(\sigma \otimes M \otimes N) \circ i_{M \otimes N} \text { (by (133)) } \\
= & \left(\omega \otimes p_{M \otimes N} \circ\left(B \otimes c_{M, B} \otimes N\right) \circ\left(\rho_{M} \otimes \rho_{N}\right) \circ c_{N, M} \circ\left(\varphi_{N} \otimes \varphi_{M}\right) \circ\left(H \otimes c_{H, N} \otimes M\right)\right. \\
& \circ((\sigma \circ \omega) \otimes N \otimes M) \circ\left(B \otimes c_{N, B} \otimes M\right) \circ\left(\rho_{N} \otimes \rho_{M}\right) \circ c_{M, N} \circ\left(\varphi_{M} \otimes \varphi_{N}\right) \circ \\
& \left.\left(H \otimes c_{H, M} \otimes N\right) \circ(\sigma \otimes M \otimes N) \circ i_{M \otimes N} \text { (by (i) of [20] [Lemma 1.4] and naturality of } c\right) \\
= & \left(\left(\omega \circ c_{B, B}\right) \otimes \omega \otimes p_{M \otimes N} \circ\left(B \otimes B \otimes B \otimes c_{M, B} \otimes N\right) \circ\left(B \otimes B \otimes \rho_{M} \otimes \rho_{N}\right)\right. \\
& \circ\left(B \otimes c_{M, B} \otimes N\right) \circ\left(\rho_{M} \otimes \rho_{N}\right) \circ\left(M \otimes \varphi_{N}\right) \circ\left(c_{H, M} \otimes N\right) \circ\left(H \otimes \varphi_{M} \otimes N\right) \\
& \circ\left(H \otimes H \otimes\left(\left(\varphi_{M} \otimes \varphi_{N}\right) \circ\left(H \otimes c_{H, M} \otimes N\right)\right)\right) \circ\left(\sigma \otimes \sigma \otimes i_{M \otimes N}\right)(\text { by the naturality of } c \text { and } \\
& (44)) \\
= & \left.\left(\left(\omega \circ c_{B, B}\right) * \omega\right) \otimes p_{M \otimes N}\right) \circ\left(B \otimes c_{M, B} \otimes N\right) \circ\left(\rho_{M} \otimes \rho_{N}\right) \circ\left(\varphi_{M} \otimes \varphi_{M}\right) \\
& \circ\left(H \otimes c_{H, M} \otimes N\right) \circ\left(\left(\left(c_{H, H} \circ \sigma\right) * \sigma\right) \otimes i_{M \otimes N}\right)(\text { by the naturality of } c \text { and the condition of H-module } \\
& \text { and left B-comodule for } M \text { and } N) \\
= & \left((\omega * \omega) \otimes p_{M \otimes N}\right) \circ\left(B \otimes c_{M, B} \otimes N\right) \circ\left(\rho_{M} \otimes \rho_{N}\right) \circ\left(\varphi_{M} \otimes \varphi_{M}\right) \circ\left(H \otimes c_{H, M} \otimes N\right) \\
& \circ\left((\sigma * \sigma) \otimes i_{M \otimes N}\right)(\text { by the triangular condition for } H \text { and the cotriangular condition for } B) \\
= & \left(\left(\varepsilon_{B} \circ \mu_{B}\right) \otimes p_{M \otimes N}\right) \circ\left(B \otimes c_{M, B} \otimes N\right) \circ\left(\rho_{M} \otimes \rho_{N}\right) \circ\left(\varphi_{M} \otimes \varphi_{M}\right) \circ\left(H \otimes c_{H, M} \otimes N\right) \\
& \circ\left(\left(\delta_{H} \circ \eta_{H}\right) \otimes i_{M \otimes N}\right)(\text { by (d5.3) of Definition } 5 \text { and (e5.3) of Definition 6) } \\
= & p_{M \otimes N} \circ \nabla \nabla_{M \otimes N} \circ i_{M \otimes N} \text { (by (120)) } \\
= & i d_{M \times N}(\text { by (132)), }
\end{aligned}
$$

and then, $\stackrel{B}{H}$ Long is symmetric.

Example 6. Let $\mathrm{G}$ be a finite groupoid such that $\mathrm{G}_{1}$ is finite. Let $\mathbb{K}$ be an algebraically closed field. Let $D$ be a quasitriangular weak Hopf monoid in the category of vector spaces over $\mathbb{K}$. Then, by Example 5 and the previous theorem, we have that the category ${ }_{D}^{B}$ Long, where $B=\mathbb{K}[G]^{*}$, is braided monoidal. As a consequence, if $\mathrm{H}$ is a finite groupoid such that $(\mathrm{G}, \mathrm{H})$ is a matched pair groupoids, the category ${ }_{D}^{B} \mathrm{Long}$, where $D=\mathbb{K}(\mathrm{G}, \mathrm{H})$, is an example of braided monoidal category. Finally, for a finite weak Hopf monoid $H$, the category ${ }_{D}^{B}$ Long, where $D$ is the Drinfel'd double of $H$, is braided monoidal.

\section{Discussion}

In this paper, we have proven that if $H$ and $B$ are weak Hopf algebras in a symmetric monoidal category where every idempotent morphism splits, the category of $H$ - $B$-Long dimodules, denoted by ${ }_{H}^{B}$ Long, is monoidal. Consequently, if $H$ is quasitriangular and $B$ coquasitriangular, we also prove that ${ }_{H}^{B}$ Long is an example of braided monoidal category. As a consequence of this result, we obtain that Long dimodules associated with weak Hopf algebras provide new solutions of the Yang-Baxter equation. In this setting the relevant facts that permit definition of the tensor product in ${ }_{H}^{B}$ Long come from the good properties 
of the image of the composition of two suitable idempotent morphisms associated with the module and comodule structure, respectively.

Finally, as mentioned in the Introduction, the results studied in this paper are strongly related with the theory developed by G. Militaru in the study of D-equation for Hopf algebras. The connection of weak Hopf algebras and the problem of to find new solutions of this equation may be the subject of on-going investigations.

Author Contributions: Conceptualization, investigation, writing original draft preparation, writing review and editing, supervision, J.N.A.Á., J.M.F.V. and R.G.R.; funding acquisition, J.N.A.Á., J.M.F.V. and R.G.R. All authors have read and agreed to the published version of the manuscript.

Funding: This research was funded by Ministerio de Economía y Competitividad of Spain: Agencia Estatal de Investigación. Unión Europea: Fondo Europeo de Desarrollo Regional. Grant MTM201679661-P: Homología, homotopía e invariantes categóricos en grupos y álgebras no asociativas.

Institutional Review Board Statement: Not applicable

Informed Consent Statement: Not applicable

Data Availability Statement: Not applicable

Conflicts of Interest: The authors declare no conflict of interest.

\section{References}

1. Long, F.W. The Brauer group of dimodule algebras. J. Algebra 1974, 31, 559-601. [CrossRef]

2. Militaru, G. The Long dimodules category and nonlinear equations. Algebr. Represent. Theory 1999, 2, 177-200. [CrossRef]

3. Wang, S.; Ding, N. New braided monoidal categories over monoidal Hom-Hopf algebras. Colloq. Math. 2017, 146, 77-97. [CrossRef]

4. Zhang, T.; Wang, S.; Wang, D. A new approach to braided monoidal categories. J. Math. Phys. 2019, 60, 013510. [CrossRef]

5. Gu, Y.; Wang, S. Hopf quasicomodules and Yetter-Drinfel'd quasicomodules. Commun. Algebra 2020, 48, 351-379. [CrossRef]

6. Nikshych, D.; Vainerman, L. Finite Quantum Groupoids and their applications. In New Directions in Hopf Algebras; Montgomery, S., Schneider, H.J., Eds.; MSRI Publications 43; Cambridge University Press: Cambridge, UK, 2002; pp. $211-262$.

7. Böhm G.; Nill, F.; Szlachányi, K. Weak Hopf algebras, I. Integral theory and C*-structure. J. Algebra 1999, 22, 385-438. [CrossRef]

8. Etingof, P.; Nikshych, D.; Ostrick, V. On fusion categories. Ann. Math. 2005, 162, 581-642. [CrossRef]

9. Hayashi, T. Face algebras IA generalization of quantum group theory. J. Math. Soc. Jpn. 1998, 50, 293-315. [CrossRef]

10. Yamanouchi, Y. Duality for generalized Kac algebras and characterization of finite groupoid algebras. J. Algebra 1994, 163, 9-50. [CrossRef]

11. Caenepeel, S.; Wang, D.; Yin, Y. Yetter-Drinfeld modules over weak Hopf algebras and the center construction. Ann. Univ. Ferrara 2005, 51, 69-98.

12. Nenciu, A. The center construction for weak Hopf algebras. Tsukuba J. Math. 2002, 26, 189-204. [CrossRef]

13. Kassel, C. Quantum Groups; GTM 155; Springer: New York, NY, USA, 1995.

14. Joyal, A.; Street, R. Braided tensor categories. Adv. Math. 1993, 102, 20-78. [CrossRef]

15. Meyer, R. Local and Analytic Cyclic Homology; EMS Tracts in Mathematics 3: Zürich, Switzerland, 2007.

16. Kadison, R.V.; Ringrose, J.R. Fundamentals of the Theory of Operator Algebras; Academic Press: New York, NY, USA, 1983; Volume I.

17. Alonso Álvarez, J.N.; Fernández Vilaboa, J.M.; González Rodríguez, R. Weak Hopf quasigroups. Asian J. Math. 2016, 20, 665-694. [CrossRef]

18. Alonso Álvarez, J.N.; González Rodríguez, R. Crossed products for weak Hopf algebras with coalgebra splitting. J. Algebra 2004, 281, 731-752. [CrossRef]

19. Alonso Álvarez, J.N.; Fernández Vilaboa, J.M.; González Rodríguez, R. Yetter-Drinfeld modules and projections of weak Hopf algebras. J. Algebra 2007, 315, 396-418. [CrossRef]

20. Alonso Álvarez, J.N.; Fernández Vilaboa, J.M.; González Rodríguez, R. Weak Hopf algebras and weak Yang-Baxter operators. J. Algebra 2008, 320, 2101-2143. [CrossRef]

21. Alonso Álvarez, J.N.; Fernández Vilaboa, J.M.; González Rodríguez, R. Weak Yang-Baxter operators and quasitriangular weak Hopf algebras. Arab. J. Sci. Eng. 2008, 33, 27-40.

22. Nikshych, D.; Turaev, V.; Vainerman, L. Invariants of knots and 3-manifolds from quantum groupoids. Topol. Appl. 2003, 127, 91-123. [CrossRef]

23. Andruskiewitsch, N.; Natale, S. Double categories and quantum groupoids. Publ. Mat. Urug. 2005, 10, 11-51.

24. Aguiar, M.; Andruskiewitsch, N. Representations of matched pairs of groupoids and applications to weak Hopf algebras. Algebr. Struct. Their Represent. 2005, 376, 127-173.

25. Alonso Álvarez, J.N.; Fernández Vilaboa, J.M.; González Rodríguez, R. Weak braided Hopf algebras. Indiana Univ. Math. J. 2008, 57, 2423-2458. [CrossRef] 